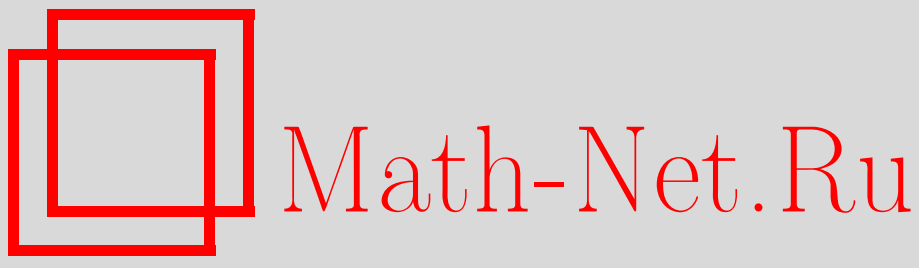

Л. Р. Волевич, А. Р. Ширикян, Локальная динамика для полулинейных гиперболических уравнений высокого порядка, Изв. РАН. Сер. матем., 2000, том 64, выпуск 3, 3-50

DOI: https://doi.org/10.4213/im288

Использование Общероссийского математического портала Math-Net.Ru подразумевает, что вы прочитали и согласны с пользовательским соглашением http://www . mathnet.ru/rus/agreement

Параметры загрузки:

IP: 44.207 .124 .84

26 апреля 2023 г., 12:12:24 
УДК 517.9

\author{
Л.Р. Волевич, А.Р. Ширикян
}

\title{
Локальная динамика для полулинейных гиперболических уравнений высокого порядка
}

Работа посвящена исследованию полулинейных гиперболических уравнений высокого порядка. Предполагается, что рассматриваемое уравнение является малым возмущением уравнения с постоянными вещественными коэффициентами, причем корни полного символа невозмущенного уравнения относительно переменной, двойственной ко времени, либо отделены от мнимой оси, либо лежат вне области $\nu<|\operatorname{Re} \tau|<\delta$, где $\delta>\nu \geqslant 0$. В первом случае доказывается, что в окрестности нуля фазовый портрет возмущенного уравнения можно линеаризовать с помощью сохраняющего время семейства гомеоморфизмов. Устанавливается также непрерывность по Гёльдеру построенных гомеоморфизмов и их обратных. Во втором случае доказывается, что в окрестности нуля фазового пространства рассматриваемого уравнения существует локально инвариантное гладкое многообразие $\mathscr{M}$, которое содержит все решения, равномерно ограниченные на временной оси, и экспоненциально притягивает решения, ограниченные на полуоси. Многообразие $\mathscr{M}$ представимо в виде графика некоторого нелинейного оператора, действующего в фазовом пространстве и являющегося малым возмущением псевдодифференциального оператора с явно выписываемым символом. При этом динамика на инвариантном многообразии $\mathscr{M}$ описывается гиперболическим уравнением, порядок которого совпадает с числом корней полного символа, лежащих в полосе $|\operatorname{Re} \tau| \leqslant \nu$.

Библиография: 34 наименования.

\section{Введение}

В теории обыкновенных дифференциальных уравнений (ОДУ) поведение решений в окрестности стационарной точки изучено достаточно подробно. Приведем хорошо известный результат Гробмана-Хартмана о линеаризации фазового портрета в окрестности гиперболической стационарной точки (см. [4], [20], [2], [10]).

Рассмотрим систему

$$
\dot{u}(t)=P u(t)+\varepsilon Q(u(t)), \quad u(t) \in \mathbb{E}
$$

где $\mathbb{E}=\mathbb{R}^{m}$ - фазовое пространство, $\varepsilon \in \mathbb{R}$ - мальй параметр, $P$ - матрища размера $m \times m$ с вещественными элементами, а $Q(u): \mathbb{R}^{m} \rightarrow \mathbb{R}^{m}$ - гладкая финитная функция, обрашающаяся в нуль при $u=0$. Рассмотрим задачу Коши для уравнения $(0.1)$ :

$$
u(0)=u_{0} \in \mathbb{R}^{m}
$$

Работа поддержана Российским фондом фундаментальных исследований (грант 97-0100541) и фондом Volkswagen-Stiftung (грант I-71016).

(C) Л.Р. Волевич, А.Р. Ширикян, 2000 
Обозначим через

$$
\mathscr{U}_{\varepsilon}(t, \cdot): \mathbb{R}^{m} \rightarrow \mathbb{R}^{m}, \quad u_{0} \mapsto u(t)
$$

разрешающий оператор для задачи $(0.1),(0.2)$. При $\varepsilon=0$ оператор $\mathscr{U}_{\varepsilon}(t, \cdot)$ является линейным и обозначается через $\mathscr{U}_{0}(t)$. Теорема Гробмана-Хартмана утверждает, что если вешественные части собственных значений матрицы $P$ отличны от нуля, то найдется такой гомеоморфизм $\Phi_{\varepsilon}: \mathbb{R}^{m} \rightarrow \mathbb{R}^{m},|\varepsilon| \ll 1$, что при всех $t \in \mathbb{R}$ и $u_{0} \in \mathbb{R}^{m}$ выполнено соотношение (см. рис. 1 , где $\left.u=\mathscr{U}_{\varepsilon}\left(t, u_{0}\right)\right)$

$$
\mathscr{U}_{0}(t) \Phi_{\varepsilon}\left(u_{0}\right)=\Phi_{\varepsilon}\left(\mathscr{U}_{\varepsilon}\left(t, u_{0}\right)\right)
$$
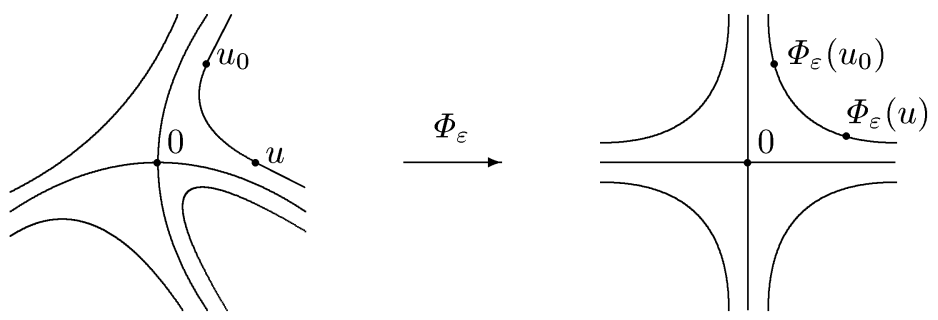

Рис. 1. Линеаризация фазового портрета

В случае, когда матрица $P$ имеет корни на мнимой оси, линеаризация, вообше говоря, невозможна, однако, как следует из результатов работы [29] (см. также [21], [33]), найдется гладкое многообразие $\mathscr{M}$ размерности $m_{c}$ (где $m_{c}-$ число чисто мнимых собственных значений матрицы $P$ ) такое, что справедливы следуюшие утверждения:

(i) любое решение, равномерно ограниченное при $t \in \mathbb{R}$, лежит на $\mathscr{M}$, при этом динамика на $\mathscr{M}$ описывается уравнением порядка $m_{c}$;

(ii) любое решение, равномерно ограниченное при $t \geqslant 0(t \leqslant 0)$, экспоненциально притягивается к некоторой траектории на $\mathscr{M}$.

Приведенные вьше результаты являются частью общей теории, посвяшенной описанию поведения решений ОДУ в окрестности стационарной точки. Подробное изложение соответствующего раздела ОДУ можно найти в [2], [6], [9], [10], [33], где, в частности, изучаются такие вопросы, как асимптотическая устойчивость, экспоненциальная дихотомия, существование и гладкость устойчивого, неустойчивого и центрального многообразий. Все эти результаты в том или ином виде переносятся на уравнения вида (0.1) с бесконечномерным фазовым пространством $\mathbb{E}$ при условии, что $P$ - ограниченный линейный оператор, а $Q(u)$ - гладкая функция в $\mathbb{E}$ (см. [5], [28]). Условие ограниченности $P$ не позволяет применить развитую абстрактную теорию к эволюционным уравнениям с частными производными, так как оператор $P$, возникающий в результате сведения таких уравнений к системам вида (0.1), представляет собой дифференщиальный оператор по пространственным переменным и поэтому неограничен. Таким образом, при изучении уравнения (0.1) в бесконечномерном фазовом пространстве "естественные" условия на оператор $P$ и нелинейную функцию $Q(u)$ диктуются конкретными примерами уравнений с 
частными производными. Упомянутые выше задачи для таких уравнений изучались многими авторами (см. работы [1], [8], [11], [14]-[18], [22]-[27], [31], [34] и цитированную там литературу).

В настоящей работе изучаются полулинейные гиперболические уравнения вида

$$
P(\partial) u(t, x)+\varepsilon Q\left(\varepsilon, t, x, \partial^{m} u(t, x)\right)=0, \quad x \in \mathbb{R}^{n}, \quad t \in \mathbb{R},
$$

где $\varepsilon \in \mathbb{R}$ - мальй параметр, $\partial=\left(\partial_{t}, \partial_{x_{1}}, \ldots, \partial_{x_{n}}\right), \partial^{m}=\left\{\partial^{\alpha}:|\alpha| \leqslant m\right\}$, a $\partial^{\alpha}=\partial_{t}^{\alpha_{0}} \partial_{x_{1}}^{\alpha_{1}} \ldots \partial_{x_{n}}^{\alpha_{n}}$. Предполагается, что оператор

$$
P(\partial)=P\left(\partial_{t}, \partial_{x}\right)=\sum_{|\alpha| \leqslant m} p_{\alpha} \partial^{\alpha}, \quad p_{\alpha} \in \mathbb{R},
$$

является строго гиперболическим, а нелинейный член $Q$ имеет вид

$$
Q\left(\varepsilon, t, x, \partial^{m} u\right)=\sum_{|\alpha| \leqslant m} q_{\alpha}(\varepsilon, t, x) \partial^{\alpha} u+q\left(\varepsilon, t, x, \partial^{m-1} u\right),
$$

где $q_{\alpha}(\varepsilon, t, x)$ и $q(\varepsilon, t, x, z), z=\left\{z_{\beta}:|\beta| \leqslant m-1\right\} \in \mathbb{R}^{d},-$ гладкие вешественнозначные функции, причем $q(\varepsilon, t, x, 0) \equiv 0$. Наряду со строгой гиперболичностью на $P(\partial)$ налагается одно из следующих условий.

УСловиЕ $(\mathrm{H})$. Найдется такое $\delta>0$, что

$$
P(\tau, \xi) \neq 0 \quad n p u \quad|\operatorname{Re} \tau|<\delta, \quad(\operatorname{Im} \tau, i \xi) \in \mathbb{R}^{n+1},
$$

где $P(\tau, \xi)$ - полный символ оператора $P\left(\partial_{t}, \partial_{x}\right)$.

УСловИЕ $\left(\mathrm{H}_{c}\right)$. Найдутся такие $\delta>\nu \geqslant 0$, что

$$
P(\tau, \xi) \neq 0 \quad \text { npu } \quad \nu<|\operatorname{Re} \tau|<\delta, \quad(\operatorname{Im} \tau, i \xi) \in \mathbb{R}^{n+1} .
$$

Другими словами, в условии $(\mathrm{H})$ предполагается, что корни полинома $P(\tau, \xi)$ отделены от мнимой оси равномерно по $i \xi \in \mathbb{R}^{n}$, тогда как в условии $\left(\mathrm{H}_{c}\right)$ допускается наличие корней символа $P(\tau, \xi)$ в окрестности мнимой оси, но требуется, чтобы они были отделены от остальных корней равномерно по $\xi$. Мы докажем, что если вьполнено условие $(\mathrm{H})$, то в произвольной конечной окрестности нуля фазовый портрет уравнения (0.3) можно линеаризовать (при достаточно малых $\varepsilon$ ) с помощью сохраняющего время семейства гомеоморфизмов. Более того, мы покажем, что если оператор $Q$ имеет порядок $m-1$, то линеаризуюшие гомеоморфизмы являются непрерывными по Гёльдеру вместе со своими обратными. Мы не приводим точные формулировки этих утверждений, отсылая читателя к п. 1.2.

В случае, когда выполнено условие $\left(\mathrm{H}_{c}\right)$, мы докажем, что в произвольной конечной окрестности нуля фазового пространства уравнения (0.3) найдется гладкое бесконечномерное многообразие $\mathscr{M}$, обладающее свойствами, аналогичными (i) и (ii) (см. выше). Более того, будет установлено, что $\mathscr{M}$ является графиком некоторого гладкого нелинейного оператора, зависяшего от $m_{c}$ функций (где $m_{c}-$ число ${ }^{1}$ корней, лежащих в полосе $\left.|\operatorname{Re} \tau| \leqslant \nu\right)$ и представляющего собой малое

\footnotetext{
${ }^{1}$ Всюду ниже мы будем предполагать, что $1 \leqslant m_{c} \leqslant m-1$.
} 
нелинейное возмущение матричного псевдодифференциального оператора, символ которого выражается через символ исходного уравнения. Точные формулировки этих утверждений приведены в п. 5.1.

Коротко опишем структуру статьи, состояшей из двух частей. Первая часть посвящена доказательству теорем типа Гробмана-Хартмана. В $\S 1$ приводятся формулировки основных результатов первой части (см. теоремы 1.3 и 1.4) и рассматриваются некоторые примеры. В $\S 2$, посвященном изучению линейных гиперболических уравнений, доказывается, что фазовые портреты уравнения с постоянными коэффициентами и его малого возмущения можно преобразовать друг в друга с помощью сохраняющего время семейства гомеоморфизмов. В $\S 3$ доказываются теоремы 1.3 и 1.4. В приложении ( $(4)$ собраны некоторые вспомогательные утверждения, использованные в основном тексте.

Вторая часть посвящена построению центрального многообразия для уравнения (0.3). В $\S 5$ приводятся формулировки и наброски доказательств основных результатов (см. теоремы 5.1-5.3). В $\S 6$ изучается начальная задача с условиями роста на бесконечности для уравнения со срезанной нелинейностью. $\S 7$ посвящен доказательству теорем 5.1-5.3. В приложении (§8) собраны некоторые вспомогательные утверждения.

Авторы выражают благодарность А.Л. Афендикову, А.Ю. Горицкому и А.И. Комечу за обсуждение полученных результатов и ценные замечания, а также М. Левитину за ТЕХническую поддержку.

ОБОЗНА ЧЕНИЯ. Пусть $0<\gamma \leqslant 1, J \subset \mathbb{R}$ - некоторый интервал, $D$ - область в $\mathbb{R}^{d}, X$ и $Y$ - банаховы пространства, а $\Omega$ - открытое подмножество в $X$. Мы будем использовать следующие пространства функций.

$C_{b}^{\infty}(D)$ - пространство бесконечно гладких функций на $D$, которые ограничены вместе со всеми производными.

$C(J, X)$ - пространство непрерывных функций на $J$ со значениями в $X$.

$C^{l, \gamma}(\Omega, Y)$ - пространство $l$ раз непрерывно дифференцируемых (по Фреше) функций $f: \Omega \rightarrow Y$, для которых $l$-я производная удовлетворяет условию Гёльдера с показателем $\gamma$, и конечна полунорма

$$
|f|_{C^{l, \gamma}}:=\sup _{u \in \Omega} \sum_{j=1}^{l}\left\|D^{j} f(u)\right\|_{\mathscr{L}^{j}(X, Y)}+\sup _{\substack{u, v \in \Omega \\ u \neq v}} \frac{\left\|D^{l} f(u)-D^{l} f(v)\right\|_{\mathscr{L}^{l}(X, Y)}}{\|u-v\|_{X}^{\gamma}}
$$

где $\mathscr{L}^{j}(X, Y)$ обозначает пространство ограниченных $j$-линейных форм из $X$ в $Y$ (см. $[11$, п. 1.2.5]). Заметим, что если область $\Omega$ ограничена, то конечна также норма

$$
\|f\|_{C^{l, \gamma}}:=\sup _{u \in \Omega}\|f(u)\|_{Y}+|f|_{C^{l, \gamma}}
$$

В случае, когда $l=0$, будем писать $C^{\gamma}(\Omega, Y)$.

Через $C_{i}, i=1,2, \ldots$, будем обозначать несущественные положительные константы. 


\section{Часть I. Теорема Гробмана-Хартмана}

\section{§ 1. Формулировки теорем о линеаризации и примеры}

В этом параграфе мы напомним теорему о корректности задачи Коши для нелинейных гиперболических уравнений (предложение 1.1), сформулируем основные результаты первой части (теоремы 1.3 и 1.4) и рассмотрим некоторые примеры.

1.1. Задача Коши для уравнения (0.3). Мы будем предполагать, что для операторов $P$ и $Q$ из уравнения (0.3) вьполнены следуюшие условия.

УСловие $(\mathrm{P})$. Оператор $P(\partial)$ имеет вид $(0.4)$ и является строго гиперболическим, т.е. он разрешен относительно старшей производной по $t$, а корни по $\tau$ старшего символа

$$
P^{0}(\tau, \xi)=\sum_{|\alpha|=m} p_{\alpha} \tau^{\alpha_{0}} \xi^{\alpha^{\prime}}, \quad \alpha=\left(\alpha_{0}, \alpha^{\prime}\right),
$$

являются чисто мнимыми и попарно различными при $i \xi \in \mathbb{R}^{n} \backslash\{0\}$.

УСловие (Q). Оператор $Q$ имеет вид (0.5), где $q_{\alpha}$ и $q$ - вещественнозначные функции, удовлетворяющие включениям $q_{\alpha} \in C_{b}^{\infty}\left([-1,1] \times \mathbb{R}_{t, x}^{n+1}\right)$ и $q \in$ $C_{b}^{\infty}\left([-1,1] \times \mathbb{R}_{t, x}^{n+1} \times B_{\rho}\right)$ для любого шара $B_{\rho}=\left\{z \in \mathbb{R}^{d}:|z| \leqslant \rho\right\}$, причем $q(\varepsilon, t, x, 0) \equiv 0$.

Рассмотрим задачу Коши для уравнения (0.3):

$$
\partial_{t}^{j} u(\theta, x)=u_{j}(x) \in H^{(m-1+k-j)}, \quad j=0, \ldots, m-1,
$$

где $k \geqslant 0$ - целое число, а $H^{(s)}=H^{(s)}\left(\mathbb{R}^{n}\right)$ - пространство Соболева порядка $s$ со стандартной нормой $\|\cdot\|_{s}$. Определим фазовое пространство уравнения (0.3) по формуле

$$
\mathbb{E}_{m-1, k}=\prod_{j=0}^{m-1} H^{(m-1+k-j)}
$$

и снабдим его нормой

$$
\|U\|_{m-1, k}=\left(\sum_{j=0}^{m-1}\left\|u_{j}\right\|_{(m-1+k-j)}^{2}\right)^{1 / 2}, \quad U=\left[u_{0}, \ldots, u_{m-1}\right] .
$$

Обозначим через $\mathbb{B}_{m-1, k}(\rho)$ открытый шар в $\mathbb{E}_{m-1, k}$ радиуса $\rho>0$ с центром в нуле.

ПРЕДЛОЖЕНИЕ 1.1. Пусть выполнены условия (P) и (Q). Тогда найдется такое $\varepsilon_{0}>0$, что при $|\varepsilon| \leqslant \varepsilon_{0}$ справедливы следующие утверждения.

(i) Для любого $\rho>0$ и челого числа $k>n / 2$ найдется такое $T>0$, что задача $(0.3),(1.1)$ с данными Коши $\left[u_{0}, \ldots, u_{m-1}\right] \in \mathbb{B}_{m-1, k}(\rho)$ имеет (притом единственное) решение $u(t, x)$, удовлетворяющее включениям

$$
\partial_{t}^{j} u \in C\left(J, H^{(m-1+k-j)}\right), \quad j=0, \ldots, m-1,
$$

əде $J=[\theta-T, \theta+T]$. 
(ii) Eсли $u_{i}(t, x), i=1,2,-$ два решения задачи (0.3), (1.1), (1.2) с $J=J_{i}$, где $J_{i} \subset \mathbb{R}, \quad i=1,2$, - замкнутье интерваль, содержащие точку $\theta$, то $u_{1}(t, x)=u_{2}(t, x)$ nрu $t \in J_{1} \cap J_{2}, x \in \mathbb{R}^{n}$.

Предложение 1.1 является одним из вариантов теоремы о корректности задачи Коши для нелинейных гиперболических уравнений. Его доказательство можно найти, например, в [7, гл. 7].

ЗАмЕчАнИЕ 1.2. Из предложения 1.1 следует, что для любых $\theta \in \mathbb{R}$ и $U_{0}=$ $\left[u_{0}, \ldots, u_{m-1}\right] \in \mathbb{E}_{m-1, k}$ найдутся такие $T_{\varepsilon}^{ \pm}=T_{\varepsilon}^{ \pm}\left(k, \theta, U_{0}\right)$, что $\left(T_{\varepsilon}^{-}, T_{\varepsilon}^{+}\right)$- максимальный интервал, на котором определено решение, соответствующее данным Коши $U_{0}$. При этом если $T_{\varepsilon}^{+}<+\infty$, то

$$
\lim _{t \rightarrow T_{\varepsilon}^{+}-0}\|\mathscr{D}(t) u\|_{m-1, k}=+\infty
$$

где через

$$
\mathscr{D}(t) u=\left[u(t, x), \partial_{t} u(t, x), \ldots, \partial_{t}^{m-1} u(t, x)\right]
$$

мы обозначили фазовую траекторию, соответствуюшую $u(t, x)$. Аналогично, если $T_{\varepsilon}^{-}>-\infty$, то

$$
\lim _{t \rightarrow T_{\varepsilon}^{-}+0}\|\mathscr{D}(t) u\|_{m-1, k}=+\infty
$$

Обозначим через

$$
\mathscr{U}_{\varepsilon}\left(t, \theta, U_{0}\right): \mathbb{E}_{m-1, k} \rightarrow \mathbb{E}_{m-1, k}, \quad|\varepsilon| \leqslant \varepsilon_{0}, \quad t \in\left(T_{\varepsilon}^{-}, T_{\varepsilon}^{+}\right)
$$

оператор, отображающий $U_{0}=\left[u_{0}, \ldots, u_{m-1}\right] \in \mathbb{E}_{m-1, k}$ в вектор-функцию (1.3), где $u(t, x)$ - решение задачи $(0.3),(1.1),(1.2)$ с $J=\left(T_{\varepsilon}^{-}, T_{\varepsilon}^{+}\right)$. При $\varepsilon=0$ уравнение $(0.3)$ является линейным, а его коэффициенты не зависят от $(t, x)$. Поэтому $T_{0}^{ \pm}= \pm \infty$, a (1.4) - обратимый линейньй оператор, зависящий только от разности $t-\theta$. Его мы будем обозначать через $\mathcal{U}_{0}(t-\theta)$.

1.2. Формулировка результатов. Напомним, что условие $(\mathrm{H})$ определено во введении.

ТЕОРема 1.3. Пусть выполнень условия (P), (Q) и (Н). Тогда для любого $\rho>0$ и произвольного иелого числа $k>n / 2$ найдутся константа $\varepsilon_{0}>0 u$ семейство непрерывных отображений

$$
\Phi_{\varepsilon}\left(\theta, U_{0}\right): \mathbb{R} \times \mathbb{B}_{m-1, k}(\rho) \rightarrow \mathbb{E}_{m-1, k}
$$

таких, что $\Phi_{\varepsilon}(\theta, 0) \equiv 0$, и при $|\varepsilon| \leqslant \varepsilon_{0}$ справедливы следующие утвержсдения.

(i) Если вектор-функиия $U_{0}=\left[u_{0}, \ldots, u_{m-1}\right] \in \mathbb{B}_{m-1, k}(\rho)$ такова, что

$$
\mathscr{U}_{\varepsilon}\left(t, \theta, U_{0}\right) \in \mathbb{B}_{m-1, k}(\rho) \quad n p u \quad t \in J
$$

әде $J \subset \mathbb{R}$ - некоторый интервал, содерэсащий точку $\theta \in \mathbb{R}$, то

$$
\mathscr{U}_{0}(t-\theta) \Phi_{\varepsilon}\left(\theta, U_{0}\right)=\Phi_{\varepsilon}\left(t, \mathscr{U}_{\varepsilon}\left(t, \theta, U_{0}\right)\right)
$$


(ii) Для произвольного фиксированного $\theta \in \mathbb{R}$ образ $\mathbb{V}_{\varepsilon}(\rho, \theta)$ открытоого иара $\mathbb{B}_{m-1, k}(\rho)$ при отображении $\Phi_{\varepsilon}(\theta, \cdot)$ является открытой окрестностью нуля в $\mathbb{E}_{m-1, k}$, причем $\Phi_{\varepsilon}(\theta, \cdot)$ гомеоморфно отображсат $\mathbb{B}_{m-1, k}(\rho)$ на $\mathbb{V}_{\varepsilon}(\rho, \theta)$.

(iii) Мнохсество $\mathbb{R}_{\theta} \times \mathbb{V}_{\varepsilon}(\rho, \theta)=\left\{\left(\theta, U_{0}\right) \in \mathbb{R} \times \mathbb{E}_{m-1, k}: U_{0} \in \mathbb{V}_{\varepsilon}(\rho, \theta)\right\}$ открыто в $\mathbb{R} \times \mathbb{E}_{m-1, k}$, а обратное отображсение

$$
\Phi_{\varepsilon}^{-1}\left(\theta, U_{0}\right): \mathbb{R}_{\theta} \times \mathbb{V}_{\varepsilon}(\rho, \theta) \rightarrow \mathbb{B}_{m-1, k}(\rho)
$$

непрерывно для любого фиксированного $\varepsilon$.

Рассмотрим теперь вопрос об эквивалентности оператора $\mathscr{U}_{\varepsilon}(t, \theta, \cdot)$ и разрешаюшего оператора $\mathscr{V}_{\varepsilon}(t, \theta)$ задачи Коши для линейного уравнения

$$
P_{\varepsilon}(t, x, \partial) u \equiv \sum_{|\alpha| \leqslant m}\left(p_{\alpha}+\varepsilon q_{\alpha}(\varepsilon, t, x)\right) \partial^{\alpha} u=0 .
$$

Таким образом, по определению линейный оператор $\mathcal{V}_{\varepsilon}(t, \theta)$, определенньй при всех $t, \theta \in \mathbb{R}$, переводит $\left[u_{0}, \ldots, u_{m-1}\right] \in \mathbb{E}_{m-1, k}$ в вектор-функцию (1.3), где $u(t, x)$ - решение задачи $(1.8),(1.1),(1.2)$ с $J=\mathbb{R}$.

Хорошо известно, что если оператор $P$ является строго гиперболическим, то корни $\tau_{j}(\xi), j=1, \ldots, m$, полного символа $P(\tau, \xi)$ лежат в некоторой полосе $|\operatorname{Re} \tau| \leqslant$ const при $i \xi \in \mathbb{R}^{n}$ (см., например, [7, гл. 4] или [32, п. 2.2]). Положим

$$
\begin{aligned}
\sigma_{\max } & =\max _{j=1, \ldots, m} \sup _{\xi \in i \mathbb{R}^{n}}\left|\operatorname{Re} \tau_{j}(\xi)\right|, \\
\sigma_{\min } & =\min _{j=1, \ldots, m} \inf _{\xi \in i \mathbb{R}^{n}}\left|\operatorname{Re} \tau_{j}(\xi)\right| .
\end{aligned}
$$

Заметим, что если оператор $P(\partial)$ удовлетворяет условию $(\mathrm{H})$, то $\sigma_{\min } \geqslant \delta>0$.

ТЕОРема 1.4. Пусть выполнены условия (P), (Q) и (Н). Тогда для любых $\rho>0 u \gamma, 0<\gamma<\sigma_{\min } / \sigma_{\max }$, и произвольного целого числа $k>n / 2$ можно указать константу $\varepsilon_{0}=\varepsilon_{0}(k, \gamma, \rho)>0$ и семейство непрерывных операторов

$$
\Psi_{\varepsilon}\left(\theta, U_{0}\right): \mathbb{R} \times \mathbb{B}_{m-1, k}(\rho) \rightarrow \mathbb{E}_{m-1, k}, \quad|\varepsilon| \leqslant \varepsilon_{0},
$$

таких, что $\Psi_{\varepsilon}(\theta, 0) \equiv 0$, и при $|\varepsilon| \leqslant \varepsilon_{0}$ справедливы следующие утвержсдения.

(i) Если вектор-функция $U_{0}=\left[u_{0}, \ldots, u_{m-1}\right] \in \mathbb{B}_{m-1, k}(\rho)$ такова, что

$$
\mathscr{U}_{\varepsilon}\left(t, \theta, U_{0}\right) \in \mathbb{B}_{m-1, k}(\rho) \quad n p u \quad t \in J,
$$

где $J \subset \mathbb{R}$ - некоторый интервал, содержащий точку $\theta \in \mathbb{R}$, то

$$
\mathscr{V}_{\varepsilon}(t, \theta) \Psi_{\varepsilon}\left(\theta, U_{0}\right)=\Psi_{\varepsilon}\left(t, \mathscr{U}_{\varepsilon}\left(t, \theta, U_{0}\right)\right) \quad n p u \quad t \in J .
$$

(ii) Для любого фиксированного $\theta \in \mathbb{R}$ образ $\mathbb{W}_{\varepsilon}(\rho, \theta)$ открытого иара $\mathbb{B}_{m-1, k}(\rho)$ при отображснии $\Psi_{\varepsilon}(\theta, \cdot)$ является открытой окрестностью нуля в $\mathbb{E}_{m-1, k}$, причем $\Psi_{\varepsilon}(\theta, \cdot)$ взаимно однозначно отображсает $\mathbb{B}_{m-1, k}(\rho)$ на $\mathbb{W}_{\varepsilon}(\rho, \theta)$. Более того, оператор $\Psi_{\varepsilon}(\theta, \cdot)$ и его обратный оператор $\Psi_{\varepsilon}^{-1}(\theta, \cdot)$ непрерывны по Гёльдеру с показателем $\gamma$.

(iii) Множсество $\mathbb{R}_{\theta} \times \mathbb{W}_{\varepsilon}(\rho, \theta)=\left\{\left(\theta, U_{0}\right) \in \mathbb{R} \times \mathbb{E}_{m-1, k}: U_{0} \in \mathbb{W}_{\varepsilon}(\rho, \theta)\right\}$ открыто в $\mathbb{R} \times \mathbb{E}_{m-1, k}$, а обратное отображсение

$$
\Psi_{\varepsilon}^{-1}\left(\theta, U_{0}\right): \mathbb{R}_{\theta} \times \mathbb{W}_{\varepsilon}(\rho, \theta) \rightarrow \mathbb{B}_{m-1, k}(\rho)
$$

непрерьвно для любого фиксированного $\varepsilon$.

Доказательство теорем 1.3 и 1.4 приведено в $\S 3$. 
ЗАмЕЧАнИЕ 1.5. Как будет видно из доказательства теорем 1.3 и 1.4 , для любого $\rho>0$ можно указать такие $\rho^{\prime}, \rho^{\prime \prime}>0$, что

$$
\mathbb{B}_{m-1, k}\left(\rho^{\prime}\right) \subset \mathbb{V}_{\varepsilon}(\rho, \theta), \quad \mathbb{W}_{\varepsilon}(\rho, \theta) \subset \mathbb{B}_{m-1, k}\left(\rho^{\prime \prime}\right),
$$

причем $\rho^{\prime} \rightarrow \infty$, когда $\rho \rightarrow \infty$. Из доказательства будет также следовать, что если нелинейный оператор $Q$ из $(0.3)$ не зависит от $t$, то линеаризуюшие операторы $\Phi_{\varepsilon}$ и $\Psi_{\varepsilon}$ не зависят от $\theta$.

1.3. Примеры. Приведем некоторые примеры строго гиперболических операторов, удовлетворяющих условию $(\mathrm{H})$.

ПримеР 1.6. Пусть $a>0, b, \sigma \in \mathbb{R} \backslash\{0\}$ и $c \in \mathbb{R}^{n}$. Положим

$$
P_{b, c}\left(\partial_{t}, \partial_{x}\right)=\partial_{t}+c \partial_{x}+b, \quad P_{a, \sigma}\left(\partial_{t}, \partial_{x}\right)=\partial_{t}^{2}+\sigma \partial_{t}+\left(1-a^{2} \Delta\right),
$$

где $\Delta$ - оператор Лапласа. Легко видеть, что операторы (1.13) являются строго гиперболическими и удовлетворяют условию $(\mathrm{H})$.

ПримеР 1.7. Пусть вектор $c \in \mathbb{R}^{n}$, вещественные числа $a_{j}, \sigma_{j}, j=1, \ldots, l$, и $b$ таковы, что $|c|<a_{j}$ при всех $j, a_{j} \neq a_{k}$ при $j \neq k$ и $b, \sigma_{j} \neq 0$. Тогда для строго гиперболических операторов

$$
\prod_{j=1}^{l} P_{a_{j}, \sigma_{j}}\left(\partial_{t}, \partial_{x}\right), \quad P_{b, c}(\partial) \prod_{j=1}^{l} P_{a_{j}, \sigma_{j}}\left(\partial_{t}, \partial_{x}\right)
$$

вьполнено условие (Н).

ПримеР 1.8. Условия $(\mathrm{P})$ и $(\mathrm{H})$ устойчивы относительно малых возмущений символа (см. [32, предложение 3.9]). Другими словами, если некоторый оператор $P(\partial)$ порядка $m$ удовлетворяет этим условиям, а $Q(\partial)$ - произвольный оператор порядка $m$ с вешественными коэффициентами, то при достаточно малых $\nu \in \mathbb{R}$ для $P+\nu Q$ выполнены условия $(\mathrm{P})$ и $(\mathrm{H})$.

Следуюший пример показывает, что оценка для показателя $\gamma$ непрерывности по Гёльдеру является точной в случае $\sigma_{\min }=\sigma_{\max }$.

ПримеР 1.9. Рассмотрим линейное ОДУ

$$
\dot{u}=-(1+\varepsilon) u \text {. }
$$

В этом случае $\sigma_{\min }=\sigma_{\max }=1$, а разрешаюший оператор задачи Коши для уравнения (1.14) имеет вид

$$
\mathscr{V}_{\varepsilon}(t) u_{0}=e^{-(1+\varepsilon) t} u_{0} .
$$

Пусть для некоторых $\varepsilon>0$ и $\rho>0$ непрерывный оператор $\Psi:[0, \rho] \rightarrow \mathbb{R}$ удовлетворяет условию

$$
\mathscr{U}_{0}(t) \Psi\left(u_{0}\right)=\Psi\left(\mathscr{U}_{\varepsilon}(t) u_{0}\right), \quad 0 \leqslant u_{0} \leqslant \rho, \quad t \geqslant 0 .
$$

Тогда, подставляя (1.15) в (1.16) и полагая $u_{0}=\rho$, получим

$$
e^{-t} \Psi(\rho)=\Psi\left(e^{-(1+\varepsilon) t} \rho\right)
$$

откуда следует, что

$$
\Psi(u)=c u^{\frac{1}{1+\varepsilon}}, \quad u>0, \quad c=\Psi(\rho) \rho^{-\frac{1}{1+\varepsilon}} .
$$

Таким образом, оператор $\Psi(u)$ непрерывен по Гёльдеру с показателем $\gamma=1 /(1+\varepsilon)$, но не удовлетворяет условию Липшица ни при каком $\varepsilon>0$. 


\section{§ 2. Эквивалентность линейных уравнений}

В этом параграфе мы покажем, что если выполнено условие (Н), то интегральные кривые уравнения (1.8) можно отобразить на интегральные кривые уравнения

$$
P(\partial) u(t, x)=0
$$

с помощью сохраняюшего время семейства гомеоморфизмов. Это утверждение будет использоваться в $\S 3$ при доказательстве теоремы 1.3.

2.1. Формулировка результата. Напомним, что операторы $\mathscr{U}_{0}(t-\theta)$ и $\mathscr{V}_{\varepsilon}(t, \theta)$ определены в пा. 1.1 и 1.2 соответственно.

ТЕОРема 2.1. Пусть выполнены условия (P), (Q) и (Н). Тогда для любого челого числа $k \geqslant 0$ можно указать константу $\varepsilon_{0}=\varepsilon_{0}(k)>0$ и семейство непрерывных отображений

$$
L_{\varepsilon}\left(\theta, U_{0}\right): \mathbb{R} \times \mathbb{E}_{m-1, k} \rightarrow \mathbb{E}_{m-1, k}, \quad|\varepsilon| \leqslant \varepsilon_{0},
$$

таких, что $L_{\varepsilon}(\theta, 0) \equiv 0$, и при $|\varepsilon| \leqslant \varepsilon_{0}$ справедливы следующие утверждения.

(i) $\mathscr{U}_{0}(t-\theta) L_{\varepsilon}\left(\theta, U_{0}\right)=L_{\varepsilon}\left(t, \mathscr{V}_{\varepsilon}(t, \theta) U_{0}\right)$ nри всех $t, \theta \in \mathbb{R} u U_{0} \in \mathbb{E}_{m-1, k}$.

(ii) Для любого фиксированного $\theta \in \mathbb{R}$ оператор $L_{\varepsilon}\left(\theta, U_{0}\right)$ гомеоморфно отображает $\mathbb{E}_{m-1, k}$ на себя. Более того, обратный оператор

$$
L_{\varepsilon}^{-1}\left(\theta, U_{0}\right): \mathbb{R}_{\theta} \times \mathbb{E}_{m-1, k} \rightarrow \mathbb{E}_{m-1, k}
$$

непрерывен по совокупности переменных $\left(\theta, U_{0}\right)$.

ЗАмЕчАнИЕ 2.2. Как будет видно из доказательства теоремы, для любого $\rho>0$ можно указать такие $\rho^{\prime}, \rho^{\prime \prime}>0$, что для образа шара $\mathbb{B}_{m-1, k}(\rho)$ под действием отображения $L_{\varepsilon}$ справедливо включение

$$
\mathbb{B}_{m-1, k}\left(\rho^{\prime}\right) \subset L_{\varepsilon}\left(\theta, \mathbb{B}_{m-1, k}(\rho)\right) \subset \mathbb{B}_{m-1, k}\left(\rho^{\prime \prime}\right),
$$

причем $\rho^{\prime} \rightarrow \infty$, когда $\rho \rightarrow \infty$. Из доказательства будет также следовать, что если коэффициенты $q_{\alpha}$ не зависят от $t$, то операторы $L_{\varepsilon}$ не зависят от $\theta$.

Теорема 2.1 будет доказана в п. 2.4. Чтобы пояснить основные идеи доказательства, в п. 2.2 мы рассмотрим частный случай, когда все корни символа $P(\tau, \xi)$ являются устойчивыми, т.е. лежат в левой полуплоскости. Кроме того, нам понадобятся некоторые результаты [3], связанные со свойством экспоненциальной дихотомии для уравнения (1.8). Они изложены в п. 2.3 .

2.2. Доказательство теоремы 2.1. Случай устойчивых корней. В этом пункте мы будем предполагать, что корни $\tau_{j}(\xi), j=1, \ldots, m$, полного символа $P(\tau, \xi)$ удовлетворяют условию

$$
\operatorname{Re} \tau_{j}(\xi) \leqslant-\sigma_{\min } \text { при } i \xi \in \mathbb{R}^{n}, \quad j=1, \ldots, m .
$$

1) Как показано в [32, теорема 6.1$]$, при $|\varepsilon| \leqslant \varepsilon_{0} \ll 1$ имеет место неравенство

$$
\left\|\mathscr{V}_{\varepsilon}(t, \theta) U_{0}\right\|_{m-1, k} \leqslant C_{1} e^{-\mu(t-\theta)}\left\|U_{0}\right\|_{m-1, k}, \quad t \geqslant \theta
$$


где $0<\mu<\sigma_{\min }$, а константа $C_{1}>0$ не зависит от $U_{0}$. Для заданных $\theta \in \mathbb{R}$ и $\varepsilon$, $|\varepsilon| \leqslant \varepsilon_{0}$, определим полунорму в $\mathbb{E}_{m-1, k}$ по формуле

$$
\left|U_{0}\right|_{(\varepsilon, \theta)}=\left(\int_{\theta}^{+\infty}\left\|\mathscr{V}_{\varepsilon}(t, \theta) U_{0}\right\|_{m-1, k}^{2} d t\right)^{1 / 2}, \quad U_{0} \in \mathbb{E}_{m-1, k}
$$

Покажем, что полунорма (2.5) эквивалентна $\|\cdot\|_{m-1, k}$. Более точно, найдется такая константа $K>1$, не зависяшая от $\varepsilon$ и $\theta$, что

$$
K^{-1}\left\|U_{0}\right\|_{m-1, k} \leqslant\left|U_{0}\right|_{(\varepsilon, \theta)} \leqslant K\left\|U_{0}\right\|_{m-1, k}, \quad U_{0} \in \mathbb{E}_{m-1, k} .
$$

В самом деле, в силу (2.4) имеем

$$
\left|U_{0}\right|_{(\varepsilon, \theta)}^{2}=\int_{\theta}^{+\infty}\left\|\mathscr{V}_{\varepsilon}(t, \theta) U_{0}\right\|_{m-1, k}^{2} d t \leqslant C_{1}^{2}(2 \mu)^{-1}\left\|U_{0}\right\|_{m-1, k}^{2} .
$$

Для доказательства обратного неравенства нам понадобится следующая лемма.

Лемма 2.3. Пусть выполнены условия (P) и (Q). Тогда найдутся такие константы $\varepsilon_{0}>0, \varkappa>0$ и $C>0$, что при $|\varepsilon| \leqslant \varepsilon_{0}$ справедливо неравенство

$$
\left\|\mathscr{V}_{\varepsilon}(t, \theta) U_{0}\right\|_{m-1, k} \leqslant C e^{\varkappa|t-\theta|}\left\|U_{0}\right\|_{m-1, k}, \quad t, \theta \in \mathbb{R}, \quad U_{0} \in \mathbb{E}_{m-1, k} .
$$

Более того, оператор $\mathscr{V}_{\varepsilon}(t, \theta) U_{0}$ непрерывен по $\left(t, \theta, U_{0}\right)$.

Неравенство (2.7) установлено в [12, лемма 23.2.1]. Непрерьвность $\mathscr{V}_{\varepsilon}$ доказывается так же, как и утверждение (iii) предложения 4.2.

В неравенстве (2.7) поменяем местами $t$ и $\theta$ и заменим $U_{0}$ на $\mathscr{V}_{\varepsilon}(t, \theta) U_{0}$. В результате получим

$$
\left\|U_{0}\right\|_{m-1, k} \leqslant C_{2}\left\|\mathscr{V}_{\varepsilon}(t, \theta) U_{0}\right\|_{m-1, k}, \quad|t-\theta| \leqslant 1,
$$

где константа $C_{2}>0$ не зависит от $\varepsilon$ и $U_{0}$. Поэтому

$$
\left|U_{0}\right|_{(\varepsilon, \theta)}^{2} \geqslant \int_{\theta}^{\theta+1}\left\|\mathscr{V}_{\varepsilon}(t, \theta) U_{0}\right\|_{m-1, k}^{2} d t \geqslant C_{2}^{-2}\left\|U_{0}\right\|_{m-1, k}^{2},
$$

что завершает доказательство (2.6).

$2)$ Обозначим через $S_{\varepsilon}(\theta)$ сферу в $\mathbb{E}_{m-1, k}$ радиуса 1 с центром в нуле относительно нормы $|\cdot|_{(\varepsilon, \theta)}$ :

$$
S_{\varepsilon}(\theta)=\left\{U_{0} \in \mathbb{E}_{m-1, k}:\left|U_{0}\right|_{(\varepsilon, \theta)}=1\right\} .
$$

Покажем, что для любого ненулевого решения $u(t, x)$ задачи $(1.8),(1.1)$ существует, притом единственньй, момент времени $T$ такой, что

$$
U(T) \in S_{\varepsilon}(T),
$$


где $U(t)=\mathscr{D}(t) u$ - фазовая траектория, соответствуюшая решению $u(t, x)$ (см. (1.3)). В самом деле, в силу неравенства

$$
|U(\theta)|_{(\varepsilon, \theta)}^{2}=\int_{\theta}^{+\infty}\|U(t)\|_{m-1, k}^{2} d t>\int_{\theta^{\prime}}^{+\infty}\|U(t)\|_{m-1, k}^{2} d t=\left|U\left(\theta^{\prime}\right)\right|_{\left(\varepsilon, \theta^{\prime}\right)}^{2},
$$

где $\theta<\theta^{\prime}$, функция $|U(\theta)|_{(\varepsilon, \theta)}$ монотонно убывает. Кроме того, из $(2.4)$ и $(2.6)$ следует, что

$$
\lim _{\theta \rightarrow+\infty}|U(\theta)|_{(\varepsilon, \theta)}=0, \quad \lim _{\theta \rightarrow-\infty}|U(\theta)|_{(\varepsilon, \theta)}=+\infty .
$$

Поэтому найдется, притом единственное, число $T \in \mathbb{R}$, удовлетворяющее условию $|U(T)|_{(\varepsilon, T)}=1$, что эквивалентно (2.8).

Для заданных $\varepsilon, \theta \in \mathbb{R}$ и $U_{0} \in \mathbb{E}_{m-1, k}$ обозначим через $T_{\varepsilon}\left(\theta, U_{0}\right)$ число $T$, удовлетворяющее условию $(2.8)$, где $U(t)=\mathscr{V}_{\varepsilon}(t, \theta) U_{0}$. Искомое отображение $(2.2)$ определим по формуле

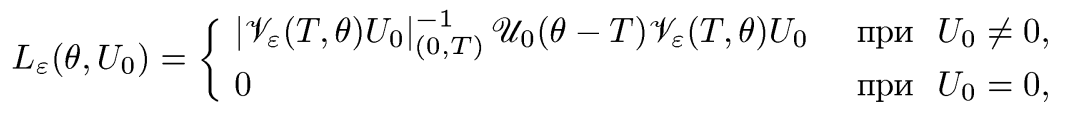

где $T=T_{\varepsilon}\left(\theta, U_{0}\right)$. Другими словами, если $U_{0} \in \mathbb{E}_{m-1, k}$ лежит на сфере $S_{\varepsilon}(\theta)$, то $L_{\varepsilon}\left(\theta, U_{0}\right)$ определяется как точка пересечения луча $\left\{\lambda U_{0}, \lambda>0\right\}$ со сферой ${ }^{2}$ $S_{0}=S_{0}(\theta)$. Если же ненулевая вектор-функция $U_{0} \in \mathbb{E}_{m-1, k}$ не лежит на $S_{\varepsilon}(\theta)$, то $L_{\varepsilon}\left(\theta, U_{0}\right)$ определяется следуюшим образом: вектор $U(T), T=T_{\varepsilon}\left(\theta, U_{0}\right)$, который лежит на траектории, проходящей через $U_{0}$ в момент времени $\theta$, и удовлетворяет (2.8), с помощью сжатия или растяжения отображается на сферу $S_{0}$, и к полученной функции применяется оператор $\mathscr{U}_{0}(\theta-T), T=T_{\varepsilon}\left(\theta, U_{0}\right)$ (см. рис. 2 , где $S_{\varepsilon}=S_{\varepsilon}(T), A=\mathscr{V}_{\varepsilon}(T, \theta) U_{0}$ и $\left.B=\left|\mathscr{V}_{\varepsilon}(T, \theta) U_{0}\right|_{(0, T)}^{-1} \mathscr{V}_{\varepsilon}(T, \theta) U_{0}\right)$.

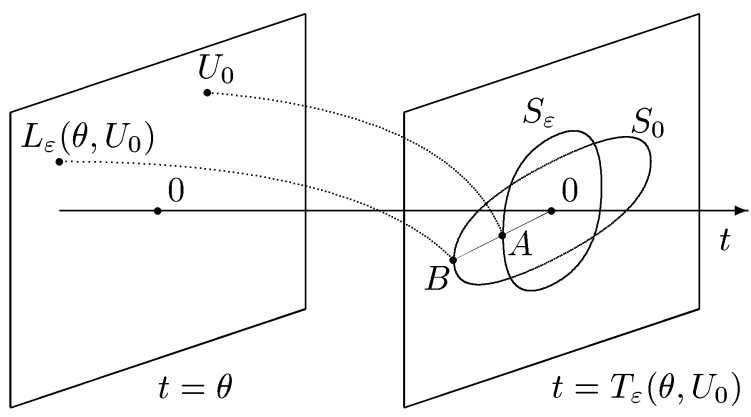

Рис. 2. Отображение $L_{\varepsilon}\left(\theta, U_{0}\right)$

3) Мы покажем, что отображение (2.9) удовлетворяет всем требуемым свойствам. Для этого нам понадобятся три вспомогательные леммы.

\footnotetext{
${ }^{2}$ При $\varepsilon=0$ норма $|\cdot|_{(\varepsilon, \theta)}$ не зависит от $\theta$, и поэтому сфера $S_{0}(\theta)$ также не зависит от $\theta$.
} 
ЛЕмма 2.4. (i) Для любого $\rho>1$ модуль разности $T_{\varepsilon}\left(\theta, U_{0}\right)-\theta$ равномерно ограничен при $\theta \in \mathbb{R}, \rho^{-1} \leqslant\left\|U_{0}\right\|_{m-1, k} \leqslant \rho$.

(ii) Найдутся такие константы $C>0 u \varkappa>0$, не зависящие от $\varepsilon u \theta$, что если $T_{\varepsilon}\left(\theta, U_{0}\right)<\theta$, то

$$
T_{\varepsilon}\left(\theta, U_{0}\right) \leqslant \theta+\varkappa^{-1} \ln \left\|U_{0}\right\|_{m-1, k}+C .
$$

ДоказАТЕльство. (i) Функция $T_{\varepsilon}\left(\theta, U_{0}\right)$ определялась как единственное решение уравнения

$$
F_{\varepsilon}\left(T, \theta, U_{0}\right):=\left|\mathscr{V}_{\varepsilon}(T, \theta) U_{0}\right|_{(\varepsilon, T)}^{2}-1=0 .
$$

Поэтому в силу (2.6) имеем

$$
K^{-1} \leqslant\left\|\mathscr{V}_{\varepsilon}(T, \theta) U_{0}\right\|_{m-1, k} \leqslant K, \quad T=T_{\varepsilon}\left(\theta, U_{0}\right)
$$

Теперь заметим, что норма $\left\|\mathscr{V}_{\varepsilon}(t, \theta) U_{0}\right\|_{m-1, k}$ стремится к нулю при $t-\theta \rightarrow+\infty$ и стремится к бесконечности при $t-\theta \rightarrow-\infty$, причем в обоих случаях сходимость является равномерной при $\rho^{-1} \leqslant\left\|U_{0}\right\|_{m-1, k} \leqslant \rho$. Отсюда и из (2.12) следует равномерная ограниченность разности $T_{\varepsilon}\left(\theta, U_{0}\right)-\theta$.

(ii) Из неравенства (2.7) следует, что

$$
\left\|\mathscr{V}_{\varepsilon}(t, \theta) U_{0}\right\|_{m-1, k} \leqslant \mathrm{C}_{2} e^{\varkappa(\theta-t)}\left\|U_{0}\right\|_{m-1, k}, \quad t \leqslant \theta .
$$

Полагая в этом неравенстве $t=T_{\varepsilon}\left(\theta, U_{0}\right)$ и пользуясь $(2.12)$, получим

$$
-\ln K \leqslant \varkappa\left(\theta-T_{\varepsilon}\left(\theta, U_{0}\right)\right)+\ln \mathrm{C}_{2}+\ln \left\|U_{0}\right\|_{m-1, k},
$$

откуда следует требуемое неравенство (2.10).

ЛЕмма 2.5. Функиия $T_{\varepsilon}\left(\theta, U_{0}\right)$ непрерывна по $\left(\theta, U_{0}\right) \in \mathbb{R} \times \mathbb{E}_{m-1, k} \backslash\{0\}$.

ДоКАЗАТЕЛЬСТво. Предположим, что последовательности $\left\{\theta_{i}\right\} \subset \mathbb{R}$ и $\left\{U_{i 0}\right\} \subset$ $\mathbb{E}_{m-1, k}$ сходятся к $\theta \in \mathbb{R}$ и $U_{0} \in \mathbb{E}_{m-1, k}$ соответственно. Положим $T_{i}=$ $T_{\varepsilon}\left(\theta_{i}, U_{i 0}\right)$. По лемме 2.4 разность $T_{i}-\theta_{i}$ ограничена равномерно по $i$. Следовательно, найдется подпоследовательность $\left\{T_{i_{k}}\right\}$, сходящаяся к некоторому пределу $T^{\prime} \in \mathbb{R}$. Полагая $T=T_{i_{k}}, \theta=\theta_{i_{k}}$ и $U_{0}=U_{i_{k} 0}$ в $(2.11)$ и переходя к пределу при $k \rightarrow \infty$, получим, что $T^{\prime}$ является решением (2.11). В силу единственности этого решения отсюда следует, что $T^{\prime}=T_{\varepsilon}\left(\theta, U_{0}\right)$ и что вся последовательность $\left\{T_{i}\right\}$ сходится к $T^{\prime}$.

Лемма 2.6. Для любих фиксированных $\varepsilon, \theta \in \mathbb{R} u U_{0} \in \mathbb{E}_{m-1, k} \backslash\{0\}$ справедливо соотношение 3

$$
T_{\varepsilon}\left(\theta, U_{0}\right)=T_{0}\left(L_{\varepsilon}\left(\theta, U_{0}\right)\right) .
$$

\footnotetext{
${ }^{3}$ При $\varepsilon=0$ функция $T_{\varepsilon}\left(\theta, U_{0}\right)$ не зависит от $\theta$, и мы обозначаем ее через $T_{0}\left(U_{0}\right)$.
} 
ДокАЗАТЕЛЬСТво. По определению $T_{0}\left(L_{\varepsilon}\left(\theta, U_{0}\right)\right)$ является единственным решением уравнения

$$
\left|\mathscr{U}_{0}(T-\theta) L_{\varepsilon}\left(\theta, U_{0}\right)\right|_{(0, T)}=1 .
$$

В силу $(2.9)$ при $T=T_{\varepsilon}\left(\theta, U_{0}\right)$ имеем

$$
\mathscr{U}_{0}(T-\theta) L_{\varepsilon}\left(\theta, U_{0}\right)=\left|\mathscr{V}_{\varepsilon}(T, \theta) U_{0}\right|_{(0, T)}^{-1} \mathscr{\mathscr { V }}_{\varepsilon}(T, \theta) U_{0}
$$

Остается заметить, что правая часть этого равенства удовлетворяет (2.14).

4) Покажем теперь, что оператор $L_{\varepsilon}\left(\theta, U_{0}\right)$, определенньй по формуле $(2.9)$, удовлетворяет всем требуемым свойствам.

Непрерывность $L_{\varepsilon}\left(\theta, U_{0}\right)$ при $U_{0} \neq 0$ является простым следствием лемм 2.3 и 2.5. Докажем, что $L_{\varepsilon}\left(\theta, U_{0}\right)$ непрерывен в точках вида $(\theta, 0)$. Для этого заметим, что если $\left\|U_{0}\right\|_{m-1, k} \ll 1$, то $T=T_{\varepsilon}\left(\theta, U_{0}\right)<\theta$, и поэтому в силу $(2.4)$ для любого $V_{0} \in \mathbb{E}_{m-1, k}$ имеет место неравенство

$$
\left\|\mathscr{U}_{0}(\theta-T) V_{0}\right\|_{m-1, k} \leqslant C_{1} e^{-\mu(\theta-T)}\left\|V_{0}\right\|_{m-1, k} .
$$

Из (2.6) следует, что

$$
\left|\mathscr{V}_{\varepsilon}(T, \theta) U_{0}\right|_{(0, T)} \geqslant K^{-1}\left\|\mathscr{V}_{\varepsilon}(T, \theta) U_{0}\right\|_{m-1, k}
$$

Подставляя $V_{0}=\mathscr{V}_{\varepsilon}(T, \theta) U_{0}$ в $(2.15)$ и учитывая $(2.16)$, придем к оценке

$$
\left\|L_{\varepsilon}\left(\theta, U_{0}\right)\right\|_{m-1, k} \leqslant C_{1} K e^{-\mu(\theta-T)} .
$$

Из (2.10) и (2.17) получим

$$
\left\|L_{\varepsilon}\left(\theta, U_{0}\right)\right\|_{m-1, k} \leqslant C_{1} K \exp \left(\mu \varkappa^{-1} \ln \left\|U_{0}\right\|_{m-1, k}+\mu C\right) \leqslant C_{3}(\varkappa, \mu)\left\|U_{0}\right\|_{m-1, k}^{\mu / \varkappa}
$$

откуда следует непрерьвность $L_{\varepsilon}\left(\theta, U_{0}\right)$ в точке $(\theta, 0)$.

Итак, закончим доказательство теоремы 2.1 для случая устойчивых корней.

ДокаЗАТЕЛЬСтво. (i) При $U_{0}=0$ требуемое соотношение тривиально. Предположим, что $U_{0} \neq 0$, и зафиксируем произвольные $t, \theta \in \mathbb{R}$. Прямая проверка показывает, что

$$
T_{\varepsilon}\left(\theta, U_{0}\right)=T_{\varepsilon}\left(t, \mathscr{V}_{\varepsilon}(t, \theta) U_{0}\right)
$$

Полагая $T=T_{\varepsilon}\left(\theta, U_{0}\right)$ и $V_{0}=\mathscr{V}_{\varepsilon}(t, \theta) U_{0}$ и пользуясь групповым свойством операторов $\mathscr{V}_{\varepsilon}$ и соотношением $(2.18)$, получим

$$
\begin{aligned}
\mathscr{U}_{0}(t-\theta) L_{\varepsilon}\left(\theta, U_{0}\right) & =\left|\mathscr{V}_{\varepsilon}(T, \theta) U_{0}\right|_{(0, T)}^{-1} \mathscr{U}_{0}(t-T) \mathscr{V}_{\varepsilon}(T, \theta) U_{0} \\
& =\left|\mathscr{V}_{\varepsilon}(T, t) V_{0}\right|_{(0, T)}^{-1} \mathscr{U}_{0}(t-T) \mathscr{V}_{\varepsilon}(T, t) V_{0} \\
& =L_{\varepsilon}\left(t, V_{0}\right) .
\end{aligned}
$$

(ii) По аналогии с (2.9) определим оператор

$$
K_{\varepsilon}\left(\theta, U_{0}\right)=\left\{\begin{array}{lll}
\left|\mathscr{U}_{0}(T-\theta) U_{0}\right|_{(\varepsilon, T)}^{-1} \mathscr{V}_{\varepsilon}(\theta, T) \mathscr{U}_{0}(T-\theta) U_{0} & \text { при } & U_{0} \neq 0, \\
0 & \text { при } & U_{0}=0,
\end{array}\right.
$$


где $T=T_{0}\left(U_{0}\right)$, и докажем, что он является обратным к $L_{\varepsilon}\left(\theta, U_{0}\right)$ для любого фиксированного $\theta$.

Положим $T=T_{\varepsilon}\left(\theta, U_{0}\right), T^{\prime}=T_{0}\left(L_{\varepsilon}\left(\theta, U_{0}\right)\right)$. В силу леммы 2.7 имеем $T=T^{\prime}$. Поэтому

$$
\begin{aligned}
\mathscr{U}_{0}\left(T^{\prime}-\theta\right) L_{\varepsilon}\left(\theta, U_{0}\right) & =\left|\mathscr{V}_{\varepsilon}(T, \theta) U_{0}\right|_{(0, T)}^{-1} \mathscr{V}_{\varepsilon}(T, \theta) U_{0}, \\
\left|\mathscr{U}_{0}\left(T^{\prime}-\theta\right) L_{\varepsilon}\left(\theta, U_{0}\right)\right|_{(\varepsilon, T)} & =\left|\mathscr{V}_{\varepsilon}(T, \theta) U_{0}\right|_{(0, T)}^{-1},
\end{aligned}
$$

откуда следует, что $K_{\varepsilon}\left(\theta, L_{\varepsilon}\left(\theta, U_{0}\right)\right)=U_{0}$. Аналогично доказьвается, что $L_{\varepsilon}(\theta$, $\left.K_{\varepsilon}\left(\theta, U_{0}\right)\right)=U_{0}$.

Таким образом, оператор $L_{\varepsilon}(\theta, \cdot)$ взаимно однозначно отображает пространство $\mathbb{E}_{m-1, k}$ на себя. Дословно повторяя использованные выше рассуждения, можно показать, что $L_{\varepsilon}^{-1}\left(\theta, U_{0}\right)=K_{\varepsilon}\left(\theta, U_{0}\right)$ непрерывен по совокупности переменных $\left(\theta, U_{0}\right)$.

2.3. Свойство экспоненциальной дихотомии. Для $\mu, \theta \in \mathbb{R}$ и целых чисел $k, l \geqslant 0$ определим пространство

$$
\mathbb{F}_{l, k,[\mu]}\left(\mathbb{R}_{ \pm}(\theta)\right)=\left\{u(t, x): e^{\mu t} \partial_{t}^{j} u \in C_{b}\left(\mathbb{R}_{ \pm}(\theta), H^{(l+k-j)}\right), j=0, \ldots, l\right\},
$$

где $\mathbb{R}_{ \pm}(\theta)=[\theta, \pm \infty)$, и снабдим его нормой

$$
E_{l, k,[\mu]}\left(u, \mathbb{R}_{ \pm}(\theta)\right)=\sup _{ \pm(t-\theta) \geqslant 0} e^{\mu(t-\theta)} E_{l, k}(u, t),
$$

где

$$
E_{l, k}^{2}(u, t)=\sum_{j=0}^{l}\left\|\partial_{t}^{j} u(t, \cdot)\right\|_{(k+l-j)}^{2} .
$$

Рассмотрим уравнение (1.8), в котором строго гиперболический оператор $P(\partial)$ удовлетворяет условию $(\mathrm{H})$. Положим $\mathbb{C}_{ \pm}(\delta):=\{\tau \in \mathbb{C}: \pm \operatorname{Re} \tau \geqslant \delta\}$ и обозначим через $m_{s}$ и $m_{u}$ число корней символа $P(\tau, \xi)$, которые лежат в полуплоскостях $\mathbb{C}_{-}\left(\sigma_{\min }\right)$ и $\mathbb{C}_{+}\left(\sigma_{\min }\right)$ соответственно. Справедливо следующее утверждение.

ПРЕДЛОЖЕНИЕ 2.7. Пусть выполнены условия (P), (Q) и (Н). Тогда для любого $\mu, 0 \leqslant \mu<\sigma_{\min }$, и челого числа $k \geqslant 0$ найдутся положительные константы $\varepsilon_{0}=\varepsilon_{0}(k, \mu)$ и $C=C(k, \mu)$ такие, что при $|\varepsilon| \leqslant \varepsilon_{0}$ справедливы следующие утверждения.

(i) Для любой вектор-функиии $U_{s}=\left[u_{0}, \ldots, u_{m_{s}-1}\right] \in \mathbb{E}_{m_{s}-1, k+m_{u}}$ уравнение (1.8) имеет, притом единственное, решение $u \in \mathbb{F}_{m-1, k,[\mu]}\left(\mathbb{R}_{+}(\theta)\right)$, удовлетворяющее начальным.м условиям

$$
\partial_{t}^{j} u(\theta, x)=u_{j}(x) \in H^{(m-1+k-j)}, \quad j=0, \ldots, m_{s}-1 .
$$

Для этого решения справедлива оченка

$$
E_{m-1, k,[\mu]}\left(u, \mathbb{R}_{+}(\theta)\right) \leqslant C \sum_{j=0}^{m_{s}-1}\left\|u_{j}\right\|_{(m-1+k-j)} .
$$


Аналогично, для любой вектор-функиии $U_{u}=\left[u_{0}, \ldots, u_{m_{u}-1}\right] \in \mathbb{E}_{m_{u}-1, k+m_{s}}$ уравнение (1.8) имеет единственное решение $u \in \mathbb{F}_{m-1, k,[-\mu]}\left(\mathbb{R}_{-}(\theta)\right)$, удовлетворяющее начальным условиям

$$
\partial_{t}^{j} u(\theta, x)=u_{j}(x) \in H^{(m-1+k-j)}, \quad j=0, \ldots, m_{u}-1 .
$$

Для этого решения справедлива оченка

$$
E_{m-1, k,[-\mu]}\left(u, \mathbb{R}_{-}(\theta)\right) \leqslant C \sum_{j=0}^{m_{u}-1}\left\|u_{j}\right\|_{(m-1+k-j)}
$$

(ii) Onepamop

$$
\mathscr{V}_{\varepsilon}^{s}(t, \theta): \mathbb{E}_{m_{s}-1, k+m_{u}} \rightarrow \mathbb{E}_{m-1, k},
$$

переводящий $U_{s}$ в вектор-функиию (1.3), где $и \in \mathbb{F}_{m-1, k,[\mu]}\left(\mathbb{R}_{+}(\theta)\right)$ - решение задачи $(1.8),(2.19)$, непрерывен по $\left(t, \theta, U_{s}\right)$. Аналогично, оператор

$$
\mathscr{V}_{\varepsilon}^{u}(t, \theta): \mathbb{E}_{m_{u}-1, k+m_{s}} \rightarrow \mathbb{E}_{m-1, k},
$$

переводящий $U_{u}$ в вектор-функцию $(1.3)$, где $и \in \mathbb{F}_{m-1, k,[-\mu]}\left(\mathbb{R}_{-}(\theta)\right)$ - решение задачи (1.8), (2.21), непрерьвен по $\left(t, \theta, U_{u}\right)$.

Утверждение (i) предложения 2.7 установлено в [3, теорема 4.1]. Непрерывность операторов (2.23) и (2.24) доказьвается так же, как утверждение (iii) предложения 4.2 (см. $\S 4$ ).

Для любого $\theta \in \mathbb{R}$ обозначим через $\mathbb{E}_{m-1, k}^{s}(\theta)$ и $\mathbb{E}_{m-1, k}^{u}(\theta)$ устойчивое и неустойчивое подпространства в $\mathbb{E}_{m-1, k}$, которые по определению состоят из вектор-функций $\left[u_{0}, \ldots, u_{m-1}\right]$, удовлетворяюших соотношениям

$$
\begin{aligned}
& {\left[u_{0}, \ldots, u_{m-1}\right]=\mathscr{V}_{\varepsilon}^{s}(\theta, \theta)\left[u_{0}, \ldots, u_{m_{s}-1}\right]} \\
& {\left[u_{0}, \ldots, u_{m-1}\right]=\mathscr{V}_{\varepsilon}^{u}(\theta, \theta)\left[u_{0}, \ldots, u_{m_{u}-1}\right]}
\end{aligned}
$$

соответственно. Следуюшее утверждение установлено в [3, теорема 5.3].

ПРЕДЛОЖЕНИЕ 2.8. В условиях предложсения 2.7 для любых $\theta \in \mathbb{R} u \varepsilon$, $|\varepsilon| \leqslant \varepsilon_{0}$, имеет место прямое разложение

$$
\mathbb{E}_{m-1, k}=\mathbb{E}_{m-1, k}^{s}(\varepsilon, \theta) \dot{+} \mathbb{E}_{m-1, k}^{u}(\varepsilon, \theta) .
$$

При этом проекторы $\mathscr{P}_{\varepsilon}^{s}(\theta)$ и $\mathscr{P}_{\varepsilon}^{u}(\theta)$, соответствующие (2.25), непрерывны по $\theta$ в сильной операторной ${ }^{4}$ топологии, а их нормы ограничены равномерно no $(\theta, \varepsilon)$.

\footnotetext{
${ }^{4}$ Напомним, что последовательность линейных отображений $L_{k}: X \rightarrow Y$ (где $X, Y$ - банаховы пространства) сходится к нулю в сильной операторной топологии, если $L_{k} u \rightarrow 0$ в $Y$ для любого $u \in X$.
} 
2.4. Доказательство теоремы 2.1. Общий случай. Приведем сначала схему доказательства. Прежде всего отметим, что оператор $\mathscr{V}_{\varepsilon}^{s}(t, \theta)$, определенный при $t \geqslant \theta$, можно продолжить на полуось $t \leqslant \theta$, полагая

$$
\mathscr{V}_{\varepsilon}^{s}(t, \theta)=\mathscr{V}_{\varepsilon}(t, \theta) \mathscr{V}_{\varepsilon}^{s}(\theta, \theta), \quad t \leqslant \theta .
$$

Аналогичным образом мы можем продолжить оператор $\mathscr{V}_{\varepsilon}^{u}(t, \theta)$ при $t \geqslant \theta$. В силу прямого разложения $(2.25)$ оператор $\mathscr{V}_{\varepsilon}(t, \theta)$ представим в виде

$$
\mathscr{V}_{\varepsilon}(t, \theta)=\mathscr{V}_{\varepsilon}^{s}(t, \theta) \mathscr{Q}_{m_{s}} \mathscr{P}_{\varepsilon}^{s}(\theta)+\mathscr{V}_{\varepsilon}^{u}(t, \theta) \mathscr{Q}_{m_{u}} \mathscr{P}_{\varepsilon}^{u}(\theta),
$$

где для $l=1, \ldots, m$ мы положили

$$
\mathscr{Q}_{l}: \mathbb{E}_{m-1, k} \rightarrow \mathbb{E}_{l-1, k+m-l}, \quad\left[u_{0}, \ldots, u_{m-1}\right] \mapsto\left[u_{0}, \ldots, u_{l-1}\right] .
$$

Первое и второе слагаемые в правой части (2.27) соответствуют динамике на семействе устойчивых и неустойчивых подпространств $\mathbb{E}_{m-1, s}^{s}(\theta)$ и $\mathbb{E}_{m-1, s}^{u}(\theta)$. Теперь заметим, что

$$
\mathscr{V}_{\varepsilon}^{s}(t, t) \mathscr{Q}_{m_{s}} U_{0}=U_{0} \text { при } U_{0} \in \mathbb{E}_{m-1, k}^{s}(t), \quad t \in \mathbb{R} .
$$

Поэтому

$$
\mathscr{V}_{\varepsilon}^{s}(t, \theta) \mathscr{Q}_{m_{s}}=\mathscr{V}_{\varepsilon}^{s}(t, t)\left[\mathscr{Q}_{m_{s}} \mathscr{V}_{\varepsilon}^{s}(t, \theta)\right] \mathscr{Q}_{m_{s}} .
$$

Таким образом, динамика на устойчивых подпространствах определяется семейством операторов $\mathscr{Q}_{m_{s}} \mathscr{V}_{\varepsilon}^{s}(t, \theta): \mathbb{E}_{m_{s}-1, k+m_{u}} \rightarrow \mathbb{E}_{m_{s}-1, k+m_{u}}$, которое экспоненциально асимптотически устойчиво при $t-\theta \rightarrow+\infty$ :

$$
\left\|\mathscr{Q}_{m_{s}} \mathscr{V}_{\varepsilon}^{s}(t, \theta) U_{s}\right\|_{m_{s}-1, k+m_{u}} \leqslant C e^{-\mu(t-\theta)}\left\|U_{s}\right\|_{m_{s}-1, k+m_{u}}, \quad t \geqslant \theta .
$$

Повторяя рассуждения, использованные в п. 2.2, мы можем построить семейство непрерывных операторов

$$
L_{\varepsilon}^{s}\left(\theta, U_{s}\right): \mathbb{R} \times \mathbb{E}_{m_{s}-1, k+m_{u}} \rightarrow \mathbb{E}_{m_{s}-1, k+m_{u}},
$$

удовлетворяюших соотношению

$$
\mathscr{Q}_{m_{s}} \mathscr{U}_{0}^{s}(t-\theta) L_{\varepsilon}^{s}\left(\theta, U_{s}\right)=L_{\varepsilon}^{s}\left(t, \mathscr{Q}_{m_{s}} \mathscr{V}_{\varepsilon}^{s}(t, \theta) U_{s}\right), \quad t, \theta \in \mathbb{R},
$$

где через $\mathscr{U}_{0}^{s}(t)$ мы обозначаем оператор $\left.\mathscr{V}_{\varepsilon}^{s}(t, 0)\right|_{\varepsilon=0}$. Аналогичное семейство

$$
L_{\varepsilon}^{u}\left(\theta, U_{u}\right): \mathbb{R} \times \mathbb{E}_{m_{u}-1, k+m_{s}} \rightarrow \mathbb{E}_{m_{u}-1, k+m_{s}}
$$

мы можем построить для операторов $\mathscr{Q}_{m_{u}} \mathscr{V}_{\varepsilon}^{u}(t, \theta)$, которые определяют динамику на неустойчивых подпространствах. Искомое отображение (2.2) определяется как "сумма" операторов $L_{\varepsilon}^{s}$ и $L_{\varepsilon}^{u}$ :

$$
L_{\varepsilon}\left(\theta, U_{0}\right)=\mathscr{U}_{0}^{s}(0) L_{\varepsilon}^{s}\left(\theta, \mathscr{Q}_{m_{s}} \mathscr{P}_{\varepsilon}^{s}(\theta) U_{0}\right)+\mathscr{U}_{0}^{u}(0) L_{\varepsilon}^{u}\left(\theta, \mathscr{Q}_{m_{u}} \mathscr{P}_{\varepsilon}^{u}(\theta) U_{0}\right),
$$

где $\mathscr{U}_{0}^{u}(t)=\left.\mathscr{V}_{\varepsilon}^{u}(t, 0)\right|_{\varepsilon=0}$. Все требуемые свойства легко проверяются.

Перейдем к подробному доказательству. Нам понадобится одно вспомогательное утверждение. 
ПРЕДЛОЖЕНИЕ 2.9. Пусть выполнены условия (P), (Q) и (Н). Тогда для любого целого числа $k \geqslant 0$ можно указать константу $\varepsilon_{0}=\varepsilon_{0}(k)>0 u$ семейство непрерывных отображений $L_{\varepsilon}^{s}(c \mathcal{M} .(2.30))$ таких, что $L_{\varepsilon}^{s}(\theta, 0) \equiv 0$ и при $|\varepsilon| \leqslant \varepsilon_{0}$ справедливы следующие утверждения.

(i) Для любых $t, \theta \in \mathbb{R} u U_{s} \in \mathbb{E}_{m_{s}-1, k+m_{u}}$ выполнено (2.31).

(ii) Для любого фиксированного $\theta \in \mathbb{R}$ оператор $L_{\varepsilon}^{s}(\theta, \cdot)$ гомеоморфно отображсает $\mathbb{E}_{m_{s}-1, k+m_{u}}$ на себя. Более того, обратный оператор

$$
K_{\varepsilon}^{s}\left(\theta, U_{s}\right): \mathbb{R} \times \mathbb{E}_{m_{s}-1, k+m_{u}} \rightarrow \mathbb{E}_{m_{s}-1, k+m_{u}}
$$

непрерывен по совокупности переменных $\left(\theta, U_{s}\right)$.

ЗАмЕчАниЕ 2.10. 1) Мы не приводим доказательство предложения 2.9, так как схема, изложенная в п. 2.2 для $\mathscr{V}_{\varepsilon}$, дословно переносится на операторы $\mathscr{Q}_{m_{s}} \mathscr{V}_{\varepsilon}^{s}$. В самом деле, доказательство теоремы 2.1 в случае устойчивых корней было основано на непрерывности оператора $\mathscr{V}_{\varepsilon}(t, \theta) U_{0}$ по $\left(t, \theta, U_{0}\right)$ и на неравенствах $(2.4)$ и (2.7). Непрерывность оператора $\mathscr{Q}_{m_{s}} \mathcal{V}_{\varepsilon}^{s}$ по совокупности переменных $\left(t, \theta, U_{s}\right)$ установлена в предложении 2.7. Неравенство (2.29) является аналогом (2.4), а из $(2.26)$ и $(2.7)$ следует, что

$$
\left\|\mathscr{Q}_{m_{s}} \mathscr{V}_{\varepsilon}^{s}(t, \theta) U_{s}\right\|_{m_{s}-1, k+m_{u}} \leqslant C e^{\varkappa(\theta-t)}\left\|U_{s}\right\|_{m_{s}-1, k+m_{u}}, \quad t \leqslant \theta .
$$

2) Аналогичное утверждение справедливо для $\mathscr{Q}_{m_{u}} \mathscr{V}_{\varepsilon}^{u}(t, \theta)$. Соответствующие операторы будем обозначать через $L_{\varepsilon}^{u}$ и $K_{\varepsilon}^{u}$.

Определим оператор $L_{\varepsilon}$ по формуле (2.33). Тогда непрерывность $L_{\varepsilon}\left(\theta, U_{0}\right)$ следует из непрерывности операторов, входящих в правую часть (2.33), а соотношение $L_{\varepsilon}(\theta, 0) \equiv 0$ очевидно.

ДокАЗАТЕЛЬСтво. (i) Применяя к (2.31) оператор $\mathscr{U}_{0}^{s}(0)$, в силу соотношения $(2.28)$, в котором $\varepsilon=0$, получим

$$
\mathscr{U}_{0}^{s}(t-\theta) L_{\varepsilon}^{s}\left(\theta, U_{s}\right)=\mathscr{U}_{0}^{s}(0) L_{\varepsilon}^{s}\left(t, \mathscr{Q}_{m_{s}} \mathscr{V}_{\varepsilon}^{s}(t, \theta) U_{s}\right) .
$$

Кроме того, заметим, что

$$
\mathscr{V}_{\varepsilon}^{s}(t, \theta) \mathscr{Q}_{m_{s}} \mathscr{P}_{\varepsilon}^{s}(\theta)=\mathscr{V}_{\varepsilon}(t, \theta) \mathscr{P}_{\varepsilon}^{s}(\theta)=\mathscr{P}_{\varepsilon}^{s}(t)^{\mathscr{V}} \varepsilon(t, \theta)
$$

Полагая $U_{s}=\mathscr{Q}_{m_{s}} \mathscr{P}_{\varepsilon}^{s}(\theta) U_{0}$ в $(2.34)$ и пользуясь $(2.26)$, придем к равенству

$$
\begin{aligned}
\mathscr{U}_{0}(t- & \theta) \mathscr{U}_{0}^{s}(0) L_{\varepsilon}^{s}\left(\theta, \mathscr{Q}_{m_{s}} \mathscr{P}_{\varepsilon}^{s}(\theta) U_{0}\right)=\mathscr{U}_{0}^{s}(t-\theta) L_{\varepsilon}^{s}\left(\theta, \mathscr{Q}_{m_{s}} \mathscr{P}_{\varepsilon}^{s}(\theta) U_{0}\right) \\
& =\mathscr{U}_{0}^{s}(0) L_{\varepsilon}^{s}\left(\theta, \mathscr{Q}_{m_{s}} \mathscr{V}_{\varepsilon}^{s}(t, \theta) \mathscr{Q}_{m_{s}} \mathscr{P}_{\varepsilon}^{s}(\theta) U_{0}\right) \\
& =\mathscr{U}_{0}^{s}(0) L_{\varepsilon}^{s}\left(\theta, \mathscr{Q}_{m_{s}} \mathscr{V}_{\varepsilon}(t, \theta) \mathscr{P}_{\varepsilon}^{s}(\theta) U_{0}\right) \\
& =\mathscr{U}_{0}^{s}(0) L_{\varepsilon}^{s}\left(\theta, \mathscr{Q}_{m_{s}} \mathscr{P}_{\varepsilon}^{s}(t) \mathscr{V}_{\varepsilon}(t, \theta) U_{0}\right) .
\end{aligned}
$$

Аналогично,

$$
\mathscr{U}_{0}(t-\theta) \mathscr{U}_{0}^{u}(0) L_{\varepsilon}^{u}\left(\theta, \mathscr{Q}_{m_{u}} \mathscr{P}_{\varepsilon}^{u}(\theta) U_{0}\right)=\mathscr{U}_{0}^{u}(0) L_{\varepsilon}^{u}\left(\theta, \mathscr{Q}_{m_{u}} \mathscr{P}_{\varepsilon}^{u}(t) \mathscr{V}_{\varepsilon}(t, \theta) U_{0}\right)
$$

Складывая (2.35) и (2.36) и учитывая (2.33), получим требуемое соотношение. 
(ii) По аналогии с (2.33) положим

$$
K_{\varepsilon}\left(\theta, U_{0}\right)=\mathscr{U}_{\varepsilon}^{s}(\theta, \theta) K_{\varepsilon}^{s}\left(\theta, \mathscr{Q}_{m_{s}} \mathscr{P}_{0}^{s} U_{0}\right)+\mathscr{U}_{\varepsilon}^{u}(\theta, \theta) K_{\varepsilon}^{u}\left(\theta, \mathscr{Q}_{m_{u}} \mathscr{P}_{0}^{u} U_{0}\right)
$$

где операторы $K_{\varepsilon}^{s}$ и $K_{\varepsilon}^{u}$ определены в предложении 2.9 и замечании 2.10 , а через $\mathscr{P}_{0}^{s}$ и $\mathscr{P}_{0}^{u}$ обозначаются проекторы $\mathscr{P}_{\varepsilon}^{s}(\theta)$ и $\mathscr{P}_{\varepsilon}^{u}(\theta)$ при $\varepsilon=0$. Легко видеть, что оператор (2.37), действующий из $\mathbb{R} \times \mathbb{E}_{m-1, k}$ в $\mathbb{E}_{m-1, k}$, является непрерывным по совокупности переменных $\left(\theta, U_{0}\right)$. Таким образом, утверждение (ii) будет установлено, если мы покажем, что $L_{\varepsilon}(\theta, \cdot)$ и $K_{\varepsilon}(\theta, \cdot)$ являются взаимно обратными отображениями для любого $\theta$.

Проверим, например, равенство

$$
K_{\varepsilon}\left(\theta, L_{\varepsilon}\left(\theta, U_{0}\right)\right)=U_{0}, \quad U_{0} \in \mathbb{E}_{m-1, k}
$$

Для этого заметим, что

$$
\mathscr{P}_{0}^{s} L_{\varepsilon}\left(\theta, U_{0}\right)=\mathscr{U}_{0}^{s}(0) L_{\varepsilon}^{s}\left(\theta, \mathscr{Q}_{m_{s}} \mathscr{P}_{\varepsilon}^{s}(\theta) U_{0}\right)
$$

Отсюда и из предложения 2.9 , (ii) и соотношения $\mathscr{Q}_{m_{s}} \mathscr{U}_{0}^{s}(0) U_{s}=U_{s}$ следует, что

$$
K_{\varepsilon}^{s}\left(\theta, \mathscr{Q}_{m_{s}} \mathscr{P}_{0}^{s} L_{\varepsilon}\left(\theta, U_{0}\right)\right)=K_{\varepsilon}^{s}\left(\theta, L_{\varepsilon}^{s}\left(\theta, \mathscr{Q}_{m_{s}} \mathscr{P}_{\varepsilon}^{s}(\theta) U_{0}\right)\right)=\mathscr{Q}_{m_{s}} \mathscr{P}_{\varepsilon}^{s}(\theta) U_{0},
$$

и поэтому в силу $(2.28)$

$$
\mathscr{V}_{\varepsilon}^{s}(\theta, \theta) K_{\varepsilon}^{s}\left(\theta, \mathscr{Q}_{m_{s}} \mathscr{P}_{0}^{s} L_{\varepsilon}\left(\theta, U_{0}\right)\right)=\mathscr{P}_{\varepsilon}^{s}(\theta) U_{0} .
$$

Аналогично,

$$
\mathscr{V}_{\varepsilon}^{u}(\theta, \theta) K_{\varepsilon}^{u}\left(\theta, \mathscr{Q}_{m_{u}} \mathscr{P}_{0}^{u} L_{\varepsilon}\left(\theta, U_{0}\right)\right)=\mathscr{P}_{\varepsilon}^{u}(\theta) U_{0}
$$

Складывая эти два соотношения, получим (2.38).

Также просто можно показать, что

$$
L_{\varepsilon}\left(\theta, K_{\varepsilon}\left(\theta, U_{0}\right)\right)=U_{0}, \quad U_{0} \in \mathbb{E}_{m-1, k}
$$

Теорема 2.1 полностью доказана.

\section{§ 3. Доказательство основных результатов}

Настояший параграф посвящен доказательству теорем 1.3 и 1.4. Мы сначала приведем одно вспомогательное утверждение о глобальной линеаризации для уравнения со срезанной нелинейностью, а затем покажем, что теоремы 1.3 и 1.4 являются простыми следствиями этого утверждения и теоремы 2.1.

3.1. Сведение теорем 1.3 и 1.4 к линеаризации для уравнения со срезанной нелинейностью. Запишем функцию $q$ из $(0.5)$ в виде

$$
q\left(\varepsilon, t, x, \partial^{m-1} u\right)=q\left(\varepsilon, t, x, \partial_{x}^{m-1} u, \partial_{x}^{m-2} \partial_{t} u, \ldots, \partial_{t}^{m-1} u\right)
$$

где $\partial_{x}^{k}=\left\{\partial_{x}^{\alpha}:|\alpha| \leqslant k\right\}$. Для заданных $\rho>0$ и $k \geqslant 0$ положим

$$
Q_{\rho}(\varepsilon, t, U)=\chi\left(\rho^{2}-\|U\|_{m-1, k}^{2}\right) q\left(\varepsilon, t, x, \partial_{x}^{m-1} u_{0}, \partial_{x}^{m-2} u_{1}, \ldots, u_{m-1}\right),
$$


где $U=\left[u_{0}, \ldots, u_{m-1}\right] \in \mathbb{E}_{m-1, k}, \chi(s) \in C^{\infty}(\mathbb{R}), \chi(s)=0$ при $s \leqslant-1$ и $\chi(s)=1$ при $s \geqslant 0$. Необходимые для дальнейшего свойства функции $Q_{\rho}$ собраны в предложении 4.1 .

Рассмотрим следующее уравнение, полученное из (0.3) заменой нелинейности $q\left(\varepsilon, t, x, \partial^{m-1} u\right)$ на $Q_{\rho}(\varepsilon, t, U(t))$, где $U(t)=\mathscr{D}(t) u$ :

$$
P_{\varepsilon}(t, x, \partial) u+\varepsilon Q_{\rho}(\varepsilon, t, U(t))=0
$$

В силу приводимого ниже предложения 4.2 при $|\varepsilon| \ll 1$ для уравнения (3.2) корректно поставлена задача Коши, т.е. для любого $\theta \in \mathbb{R}$ и произвольной вектор-функции $\left[u_{0}, \ldots, u_{m-1}\right] \in \mathbb{E}_{m-1, k}$ существует, притом единственное, решение уравнения (3.2), которое удовлетворяет включениям

$$
\partial_{t}^{j} u \in C\left(\mathbb{R}, H^{(m-1+k-j)}\right), \quad j=0, \ldots, m-1,
$$

и начальным условиям

$$
\partial_{t}^{j} u(\theta, x)=u_{j}(x) \in H^{(m-1+k-j)}, \quad j=0, \ldots, m-1
$$

Обозначим через

$$
\mathscr{U}_{\varepsilon}^{\rho}\left(t, \theta, U_{0}\right): \mathbb{R}_{t} \times \mathbb{R}_{\theta} \times \mathbb{E}_{m-1, k} \rightarrow \mathbb{E}_{m-1, k}
$$

разрешающий процесс задачи (3.2)-(3.4) (см. предложение 4.2).

Для доказательства теорем 1.3 и 1.4 нам понадобится следуюшее утверждение. Напомним, что числа $\sigma_{\min }$ и $\sigma_{\max }$ определены в $(1.9)$.

ТЕОРема 3.1. Пусть выполнены условия (P), (Q) $и(\mathrm{H})$. Тогда для любьх $\rho>0$ и $\gamma, 0<\gamma<\sigma_{\min } / \sigma_{\max }$, и произвольного целого числа $k>n / 2$ можно указать константу $\varepsilon_{0}=\varepsilon_{0}(k, \gamma, \rho)>0$ и семейство непрерывных операторов

$$
N_{\varepsilon, \rho}\left(\theta, U_{0}\right): \mathbb{R} \times \mathbb{E}_{m-1, k} \rightarrow \mathbb{E}_{m-1, k}, \quad|\varepsilon| \leqslant \varepsilon_{0},
$$

таких, что $N_{\varepsilon, \rho}(\theta, 0) \equiv 0$, и при $|\varepsilon| \leqslant \varepsilon_{0}$ справедливы следуюшие утверәсдения.

(i) $\mathscr{V}_{\varepsilon}(t, \theta) N_{\varepsilon, \rho}\left(\theta, U_{0}\right)=N_{\varepsilon, \rho}\left(t, \mathscr{U}_{\varepsilon}^{\rho}\left(t, \theta, U_{0}\right)\right)$ nрu всех $t, \theta \in \mathbb{R} u U_{0} \in \mathbb{E}_{m-1, k}$.

(ii) Для любого фиксированного $\theta \in \mathbb{R}$ оператор $N_{\varepsilon, \rho}(\theta, \cdot)$ взаимно однозначно отображсает $\mathbb{E}_{m-1, k}$ на себя. Более того, оператор $N_{\varepsilon, \rho}(\theta, \cdot)$ и его обратный $N_{\varepsilon, \rho}^{-1}(\theta, \cdot)$ непрерывны по Гёльдеру с показателем $\gamma$.

(iii) Обратный оператор $N_{\varepsilon, \rho}^{-1}\left(\theta, U_{0}\right): \mathbb{R}_{\theta} \times \mathbb{E}_{m-1, k} \rightarrow \mathbb{E}_{m-1, k}$ непрерывен по совокупности переменных $\left(\theta, U_{0}\right)$.

Теорема 3.1 будет доказана в пп. 3.2 и 3.3 . 
ДОКАЗАТЕЛЬСТВО ТЕОРЕМЫ 1.4. Зафиксируем произвольные $\rho>0$ и $\gamma, 0<$ $\gamma<\sigma_{\min } / \sigma_{\max }$, и целое число $k>n / 2$ и выберем константу $\varepsilon_{0}>0$ настолько малой, чтобы при $|\varepsilon| \leqslant \varepsilon_{0}$ выполнялось утверждение теоремы 3.1. Определим оператор $\Psi_{\varepsilon}$ как сужение $N_{\varepsilon, \rho}$ на множество $\mathbb{R} \times \mathbb{B}_{m-1, k}(\rho)$ :

$$
\Psi_{\varepsilon}\left(\theta, U_{0}\right)=N_{\varepsilon, \rho}\left(\theta, U_{0}\right), \quad \theta \in \mathbb{R}, \quad U_{0} \in \mathbb{B}_{m-1, k}(\rho) .
$$

Очевидно, что $\Psi_{\varepsilon}(\theta, 0) \equiv 0$ и что оператор $\Psi_{\varepsilon}\left(\theta, U_{0}\right)$ непрерывен по совокупности переменных и обратим для любого фиксированного $\theta$, причем обратный оператор $\Psi_{\varepsilon}^{-1}$ совпадает с сужением $N_{\varepsilon, \rho}^{-1}$ на образ $\mathbb{W}_{\varepsilon}(\theta, \rho)$ шара $\mathbb{B}_{m-1, k}(\rho)$ под действием $N_{\varepsilon, \rho}(\theta, \cdot)$. Следовательно, $\Psi_{\varepsilon}^{-1}\left(\theta, U_{0}\right)$ также непрерывен по совокупности переменных. Так как уравнения (0.3) и (3.2) совпадают в шаре $\mathbb{B}_{m-1, k}(\rho)$, то утверждение (i) следует из теоремы $3.1,(\mathrm{i})$. Множество $\mathbb{W}_{\varepsilon}(\theta, \rho)$, как образ открытого шара под действием гомеоморфизма, является открытым. Непрерывность по Гёльдеру оператора $\Psi_{\varepsilon}(\theta, \cdot)$ и его обратного следует из аналогичных свойств для $N_{\varepsilon, \rho}$. Остается заметить, что множество $\mathbb{R}_{\theta} \times \mathbb{W}_{\varepsilon}(\theta, \rho)$ является прообразом открытого шара $\mathbb{B}_{m-1, k}(\rho)$ под действием непрерывного отображения $N_{\varepsilon, \rho}^{-1}$ и поэтому открыто.

ДоКАЗАТЕЛЬСТво ТЕОРЕМЫ 1.3. Зафиксируем произвольные $\rho>0$ и $\gamma, 0<$ $\gamma<\sigma_{\min } / \sigma_{\max }$, и целое число $k>n / 2$ и выберем константу $\varepsilon_{0}>0$ настолько малой, чтобы при $|\varepsilon| \leqslant \varepsilon_{0}$ вьполнялись утверждения теорем 2.1 и 3.1. Определим оператор $\Phi_{\varepsilon}$ как сужение композиции $L_{\varepsilon}$ и $N_{\varepsilon, \rho}$ на множество $\mathbb{R} \times \mathbb{B}_{m-1, k}(\rho)$ :

$$
\Phi_{\varepsilon}\left(\theta, U_{0}\right)=L_{\varepsilon}\left(\theta, N_{\varepsilon, \rho}\left(\theta, U_{0}\right)\right), \quad \theta \in \mathbb{R}, \quad U_{0} \in \mathbb{B}_{m-1, k}(\rho) .
$$

Как и в случае теоремы 4.1, все требуемые свойства легко проверяются.

ЗАмЕчАниЕ 3.2. Из доказательства теоремы 3.1 будет следовать, что оператор $N_{\varepsilon, \rho}$ Удовлетворяет неравенству

$$
\left\|N_{\varepsilon, \rho}\left(\theta, U_{0}\right)-U_{0}\right\|_{m-1, k} \leqslant \text { const } \ll 1 \text { при всех } \theta \in \mathbb{R}, \quad U_{0} \in \mathbb{E}_{m-1, k} .
$$

На примере обыкновенных дифференциальных уравнений покажем, что в классе операторов, удовлетворяюших условию (3.6), оценка показателя Гёльдера $\gamma$ является точной. А именно рассмотрим систему

$$
\begin{aligned}
& \dot{u}_{1}=u_{1}+\varepsilon q\left(u_{2}\right), \\
& \dot{u}_{2}=u_{3}, \\
& \dot{u}_{3}=4 u_{3}+4 u_{2},
\end{aligned}
$$

где $\varepsilon>0$,

$$
q \in C_{0}^{\infty}(\mathbb{R}), \quad q \geqslant 0, \quad q(u)=u^{2} \text { при }|u| \leqslant 1 .
$$

В этом случае $\sigma_{\min }=1$ и $\sigma_{\max }=2$. Обозначим через $\mathscr{U}_{\varepsilon}\left(t, u^{0}\right): \mathbb{R}^{3} \rightarrow \mathbb{R}^{3}$, $t \in \mathbb{R}, u^{0} \in \mathbb{R}$, разрешаюший оператор задачи Коши для системы (3.7). Как показано в $[30, \S 4]$, при $|\varepsilon| \ll 1$ сушествует, притом единственный, гомеоморфизм $N: \mathbb{R}^{3} \rightarrow \mathbb{R}^{3}$, удовлетворяюший условиям

$$
\begin{gathered}
\mathscr{U}_{0}(t) N\left(u^{0}\right)=N\left(\mathscr{U}_{\varepsilon}\left(t, u^{0}\right)\right), \quad t \in \mathbb{R}, \quad u^{0} \in \mathbb{R}^{3}, \\
\left|N\left(u^{0}\right)-u^{0}\right| \leqslant \mathrm{const} \ll 1, \quad u^{0} \in \mathbb{R}^{3},
\end{gathered}
$$


где через $|\cdot|$ обозначена норма в $\mathbb{R}^{3}$. Покажем, что $N$ не удовлетворяет условию Гёльдера с показателем $\gamma=1 / 2$. В самом деле, согласно [2, $\S 34]$ оператор $N$ имеет вид

$$
N\left(u^{0}\right)=u^{0}+\varepsilon \int_{0}^{+\infty} \mathscr{U}_{0}(-\tau)\left[q\left(u_{2}\left(\tau, u^{0}\right)\right), 0,0\right] d \tau
$$

где $u_{2}\left(\tau, u^{0}\right)$ - вторая компонента вектор-функции $\mathscr{U}_{\varepsilon}\left(t, u^{0}\right)$. В частности, полагая $u^{0}=(1 / 2)[0,0, v], 0<v \ll 1$, для первой компоненты $(3.9)$ получим

$$
N_{1}\left(u^{0}\right)=\varepsilon \int_{0}^{+\infty} e^{-\tau} q\left(\tau e^{2 \tau} v\right) d \tau
$$

Предположим, что $N$ удовлетворяет условию Гёльдера с показателем $\gamma=\frac{1}{2}$. Тогда

$$
\left|N_{1}\left(u^{0}\right)\right| \leqslant \text { const }|v|^{1 / 2} .
$$

$\mathrm{C}$ другой стороны, обозначим через $h(v)>0$ единственное решение уравнения $\tau e^{2 \tau} v=1$. Легко видеть, что

$$
h(v)+\frac{1}{2} \ln v \rightarrow+\infty \text { при } v \rightarrow+0 .
$$

Поэтому в силу (3.8) имеем

$$
N_{1}\left(u^{0}\right) \geqslant \varepsilon \int_{0}^{h(v)} e^{-\tau}\left(\tau e^{2 \tau} v\right)^{2} d \tau \geqslant \frac{\varepsilon}{6}(v h(v))^{1 / 2} .
$$

Полученная оценка противоречит (3.10).

3.2. Доказательство теоремы 3.1. Нам понадобится одно вспомогательное утверждение (см. ниже предложение 3.3). Чтобы сформулировать его, рассмотрим уравнение $(3.2)$, в котором функция $Q_{\rho}$ заменена на другую функцию $R_{\rho}$ вида (3.1):

$$
P_{\varepsilon}(t, x, \partial) v+\varepsilon R_{\rho}(\varepsilon, t, V(t))=0 .
$$

Здесь $V(t)=V(t, \cdot)=\mathscr{D}(t) v$,

$$
R_{\rho}(\varepsilon, t, V)=\chi\left(\rho^{2}-\|V\|_{m-1, k}^{2}\right) r\left(\varepsilon, t, x, \partial_{x}^{m-1} v_{0}, \partial_{x}^{m-2} v_{1}, \ldots, v_{m-1}\right),
$$

где $V=\left[v_{0}, \ldots, v_{m-1}\right] \in \mathbb{E}_{m-1, k}$, а $r(\varepsilon, t, x, z)$ - гладкая функция. Как и в случае уравнения (3.2), мы будем рассматривать решения уравнения (3.12), удовлетворяюшие включениям

$$
\partial_{t}^{j} v \in C\left(\mathbb{R}, H^{(m-1+k-j)}\right), \quad j=0, \ldots, m-1 .
$$

Для заданного $\mu \geqslant 0$ и целых чисел $k, l \geqslant 0$ определим пространство

$$
\mathbb{F}_{l, k,[\mu,-\mu]}=\left\{u(t, x): e^{-\mu|t|} \partial_{t}^{j} u \in C_{b}\left(\mathbb{R}, H^{(l+k-j)}\right), j=0, \ldots, l\right\},
$$

снабженное нормой

$$
E_{l, k,[\mu,-\mu]}(u)=\sup _{t \in \mathbb{R}} e^{-\mu|t|} E_{l, k}(u, t) .
$$

В случае $\mu=0$ вместо $\mathbb{F}_{l, k,[\mu,-\mu]}$ и $E_{l, k,[\mu,-\mu]}(u)$ будем писать $\mathbb{F}_{l, k}$ и $E_{l, k}(u)$. 
ПРЕДЛОЖЕНИЕ 3.3. Пусть выполнены условия (P), (Q) u (Н), и пусть функция $r(\varepsilon, t, x, z)$ из (3.13) удовлетворяет тем же условиям, что и $q(\varepsilon, t, x, z)$. Тогда для любых $\rho>0, \quad \varkappa>\sigma_{\max } u \mu, 0<\mu<\sigma_{\min }, u$ произвольного челого числа $k>n / 2$ можно указать константы $\varepsilon_{0}>0$ и $C>0$ такие, что при $|\varepsilon| \leqslant \varepsilon_{0}$ справедливы следующие утверждения.

(i) Для любых $\theta \in \mathbb{R} u U_{0}=\left[u_{0}, \ldots, u_{m-1}\right] \in \mathbb{E}_{m-1, k}$ существует, притом единственное, решение $v(t, x)=: \mathscr{F}_{Q_{\rho}, R_{\rho}}\left(\theta, U_{0}\right)$ задачи (3.12), (3.14) такое, чmo

$$
v \in \mathbb{F}_{m-1, k,[\varkappa,-\varkappa]}, \quad u-v \in \mathbb{F}_{m-1, k}
$$

где $и(t, x)$ - решение задачи (3.2)-(3.4).

(ii) Пусть $v_{i}=\mathscr{F}_{Q_{\rho}, R_{\rho}}\left(\theta_{i}, U_{i 0}\right), \quad i=1,2$, дде $\theta_{i} \in \mathbb{R} u U_{i 0} \in \mathbb{E}_{m-1, k}$. Тогда

$$
E_{m-1, k,[\varkappa,-\varkappa]}\left(v_{1}-v_{2}\right) \leqslant C\left(\left\|U_{10}-U_{20}\right\|_{m-1, k}+\left\|U_{10}-U_{20}\right\|_{m-1, k}^{\gamma}\right),
$$

əде $\gamma=\mu / \varkappa$.

(iii) Пусть последовательности $\left\{\theta_{i}\right\} \subset \mathbb{R} u\left\{U_{i 0}\right\} \subset \mathbb{E}_{m-1, k}$ сходятся $\kappa$ $\theta \in \mathbb{R} u U_{0} \in \mathbb{E}_{m-1, k}$ соответственно, и пусть $v_{i}=\mathscr{F}_{Q_{\rho}, R_{\rho}}\left(\theta_{i}, U_{i 0}\right), \quad v=$ $\mathscr{F}_{Q_{\rho}, R_{\rho}}\left(\theta, U_{0}\right)$. Тогда

$$
E_{m-1, k,[\varkappa,-\varkappa]}\left(v_{i}-v\right) \rightarrow 0 \quad n p u \quad i \rightarrow \infty .
$$

Предполагая предложение 3.3 установленным, завершим ${ }^{5}$ доказательство теоремы 3.1 .

Определим искомое отображение (3.5) по формуле

$$
N_{\varepsilon, \rho}\left(\theta, U_{0}\right)=\mathscr{D}(\theta)\left(\left.\mathscr{F}_{Q_{\rho}, R_{\rho}}\left(\theta, U_{0}\right)\right|_{R_{\rho} \equiv 0}\right),
$$

где оператор $\mathscr{D}(\theta)$ определен в (1.3). Соотношение $N_{\varepsilon, \rho}(\theta, 0) \equiv 0$ очевидно. В силу утверждения (iii) предложения 3.3 оператор $N_{\varepsilon, \rho}$ непрерывен по совокупности переменных $\left(\theta, U_{0}\right)$.

ДокаЗАТЕльСтво (i). Зафиксируем произвольные $\theta$ и $U_{0}=\left[u_{0}, \ldots, u_{m-1}\right] \in$ $\mathbb{E}_{m-1, k}$ и обозначим через $u(t, x)$ решение задачи (3.2)-(3.4). Положим $v(t, x)=$ $\left.\mathscr{F}_{Q_{\rho}, R_{\rho}}\left(\theta, U_{0}\right)\right|_{R_{\rho} \equiv 0}$. Пусть $U(t)$ и $V(t)$ - фазовые траектории, соответствуюшие решениям $u(t, x)$ и $v(t, x)$. Из утверждения (i) предложения 3.3 вытекает, что

$$
v=\left.\mathscr{F}_{Q_{\rho}, R_{\rho}}(t, U(t))\right|_{R_{\rho} \equiv 0}, \quad t \in \mathbb{R},
$$

и поэтому

$$
V(t)=\mathscr{D}(t)\left(\left.\mathscr{F}_{Q_{\rho}, R_{\rho}}\left(\theta, U_{0}\right)\right|_{R_{\rho} \equiv 0}\right)=\mathscr{D}(t)\left(\left.\mathscr{F}_{Q_{\rho}, R_{\rho}}(t, U(t))\right|_{R_{\rho} \equiv 0}\right) .
$$

Отсюда следует требуемое соотношение.

\footnotetext{
${ }^{5}$ Мы повторяем рассуждения, использованные в [28].
} 
ДокАЗАТЕЛЬСтво (ii) и (iii). Простая проверка показывает, что оператор

$$
N_{\varepsilon, \rho}^{-1}\left(\theta, U_{0}\right):=\mathscr{D}(\theta)\left(\mathscr{F} R_{\rho},\left.Q_{\rho}\left(\theta, U_{0}\right)\right|_{R_{\rho} \equiv 0}\right)
$$

является обратньм к $N_{\varepsilon, \rho}(\theta, \cdot)$ для любого фиксированного $\theta \in \mathbb{R}$. Поэтому непрерывность по Гёльдеру операторов $N_{\varepsilon, \rho}(\theta, \cdot)$ и $N_{\varepsilon, \rho}^{-1}(\theta, \cdot)$ следует из неравенства (3.18), а непрерывность $N_{\varepsilon, \rho}^{-1}$ по совокупности переменных $\left(\theta, U_{0}\right)$ - из утверждения (iii) предложения 3.3 .

3.3. Доказательство предложения 3.3. (i) Функцию $v(t, x)$ будем искать в виде

$$
v=u+w, \quad w \in \mathbb{F}_{m-1, k} .
$$

Подставляя (3.20) в (3.12) и учитывая $(3.2)$, для $w(t, x)$ получим уравнение

$$
P_{\varepsilon}(t, x, \partial) w=\varepsilon M(U, W), \quad M(U, W)=Q_{\rho}(\varepsilon, t, U)-R_{\rho}(\varepsilon, t, U+W),
$$

где $U=\mathscr{D}(t) u, W=\mathscr{D}(t) w$. Методом сжимающих отображений докажем, что при $|\varepsilon| \ll 1$ уравнение (3.21) однозначно разрешимо в пространстве $\mathbb{F}_{m-1, k}$.

Рассмотрим оператор $A$, переводяший функцию $z \in \mathbb{F}_{m-1, k}$ в решение $w \in$ $\mathbb{F}_{m-1, k}$ уравнения

$$
P_{\varepsilon}(t, x, \partial) w=\varepsilon M(U, Z), \quad Z=\mathscr{D}(t) z .
$$

Проверим, что оператор $A$ корректно определен и переводит пространство $\mathbb{F}_{m-1, k}$ в себя. В самом деле, в силу предложения 4.1 , (i) если $z \in \mathbb{F}_{m-1, k}$, то $M(U, Z) \in$ $\mathbb{F}_{0, k}$. Поэтому согласно предложению 4.3 , в котором $\mu=0$, при $|\varepsilon| \ll 1$ уравнение (3.22) имеет, притом единственное, решение $w \in \mathbb{F}_{m-1, k}$.

Покажем теперь, что $A$ является сжимаюшим отображением. Пусть $w_{i}=$ $A\left(z_{i}\right), i=1,2$. Тогда функция $w=w_{1}-w_{2} \in \mathbb{F}_{m-1, k}$ удовлетворяет уравнению

$$
P_{\varepsilon}(t, x, \partial) w=\varepsilon\left(M\left(U, Z_{2}\right)-M\left(U, Z_{1}\right)\right),
$$

где $Z_{i}=\mathscr{D}(t) z_{i}, i=1,2$. Поэтому согласно неравенству (4.8) с $\mu=\theta=0$

$$
E_{m-1, k}(w) \leqslant C_{1}|\varepsilon| E_{0, k}\left(M\left(U, Z_{1}\right)-M\left(U, Z_{2}\right)\right) .
$$

Применяя к правой части (3.23) неравенство (4.2) с $\gamma=1$, получим

$$
E_{0, k}\left(M\left(U, Z_{1}\right)-M\left(U, Z_{2}\right)\right) \leqslant C_{2} E_{m-1, k}\left(z_{1}-z_{2}\right) .
$$

Сопоставляя эту оценку с (3.23), придем к неравенству

$$
E_{m-1, k}\left(A\left(z_{1}\right)-A\left(z_{2}\right)\right) \leqslant C_{1} C_{2}|\varepsilon| E_{m-1, k}\left(z_{1}-z_{2}\right) .
$$

Отсюда следует, что отображение $A(z)$ является сжимаюшим.

Таким образом, мы установили сушествование и единственность решения $w \in$ $\mathbb{F}_{m-1, k}$ уравнения (3.21). Отсюда следует требуемое утверждение.

(ii) Прежде всего покажем, что если $v_{i}=\mathscr{F}_{Q_{\rho}, R_{\rho}}\left(\theta, U_{i 0}\right), i=1,2$, то

$$
E_{m-1, k,[\mu,-\mu]}\left(S(\theta)\left(w_{1}-w_{2}\right)\right) \leqslant C_{3}\left\|U_{10}-U_{20}\right\|_{m-1, k}^{\gamma},
$$


где $w_{i}=v_{i}-u_{i}$, а $u_{i}(t, x)$ - решение задачи $(3.2)-(3.4)$ с данными Коши $U_{i 0} . \mathrm{B}$ самом деле, положим $w=w_{1}-w_{2}, U_{i}=\mathscr{D}(t) u_{i}, W_{i}=\mathscr{D}(t) w_{i}$. Тогда функция $w(t, x) \in \mathbb{F}_{m-1, k,[\mu,-\mu]}$ является решением уравнения

$$
P_{\varepsilon}(t, x, \partial) w=\varepsilon h(t, x), \quad h(t, x)=R_{\rho}\left(\varepsilon, t, U_{2}+W_{2}\right)-R_{\rho}\left(\varepsilon, t, U_{1}+W_{1}\right) .
$$

В силу неравенства (4.2)

$$
\|h(t, \cdot)\|_{k} \leqslant C_{4}\left(\left\|U_{2}(t, \cdot)-U_{1}(t, \cdot)\right\|_{m-1, k}^{\gamma}+\left\|W_{2}(t, \cdot)-W_{1}(t, \cdot)\right\|_{m-1, k}\right),
$$

откуда следует, что $h \in \mathbb{F}_{0, k,[\mu,-\mu]}$, причем

$$
\begin{aligned}
E_{0, k,[\mu,-\mu]}(S(\theta) h) \leqslant & C_{4} \sup _{t \in \mathbb{R}}\left(e ^ { - \mu | t - \theta | } \left[\left\|U_{2}(t, \cdot)-U_{1}(t, \cdot)\right\|_{m-1, k}^{\gamma}\right.\right. \\
& \left.\left.+\left\|W_{2}(t, \cdot)-W_{1}(t, \cdot)\right\|_{m-1, k}\right]\right) \\
\leqslant & C_{4}\left(E _ { m - 1 , k , [ \varkappa , - \varkappa ] } ^ { \gamma } \left(S(\theta)\left(u_{2}-u_{1}\right)\right.\right. \\
& \left.\left.+E_{m-1, k,[\mu,-\mu]}(S(\theta) w)\right)\right) .
\end{aligned}
$$

По неравенству (4.6) имеем

$$
E_{m-1, k,[\varkappa,-\varkappa]}\left(S(\theta)\left(u_{2}-u_{1}\right)\right) \leqslant K_{2}\left\|U_{20}-U_{10}\right\|_{m-1, k} .
$$

Подставляя эту оценку в $(3.26)$, получим

$$
E_{0, k,[\mu,-\mu]}(S(\theta) h) \leqslant C_{5}\left(\left\|U_{20}-U_{10}\right\|_{m-1, k}^{\gamma}+E_{m-1, k,[\mu,-\mu]}(S(\theta) w)\right) .
$$

Согласно предложению 4.3 решение $w \in \mathbb{F}_{m-1, k,[\mu,-\mu]}$ уравнения (3.25) удовлетворяет неравенству

$$
E_{m-1, k,[\mu,-\mu]}(S(\theta) w) \leqslant K_{3}|\varepsilon| E_{0, k,[\mu,-\mu]}(S(\theta) h) .
$$

Сопоставляя (3.28) и (3.29), придем к неравенству

$$
E_{m-1, k,[\mu,-\mu]}(S(\theta) w) \leqslant K_{3} C_{5}|\varepsilon|\left(\left\|U_{20}-U_{10}\right\|_{m-1, k}^{\gamma}+E_{m-1, k,[\mu,-\mu]}(S(\theta) w)\right),
$$

откуда при $|\varepsilon| \ll 1$ следует (3.24).

Докажем теперь (3.18). В силу представления (3.20) для функций $v_{i}, i=1,2$, имеем

$$
\begin{aligned}
E_{m-1, k,[\varkappa,-\varkappa]} & \left.S(\theta)\left(v_{1}-v_{2}\right)\right) \\
& \leqslant E_{m-1, k,[\varkappa,-\varkappa]}\left(S(\theta)\left(u_{1}-u_{2}\right)\right)+E_{m-1, k,[\mu,-\mu]}\left(S(\theta)\left(w_{1}-w_{2}\right)\right) .
\end{aligned}
$$

Поэтому требуемая оценка следует из (3.24) и (3.27).

(iii) Обозначим через $u(t, x)$ решение задачи (3.2)-(3.4), отвечающее данным Коши $U_{0}$, а через $u_{i}(t, x)$ - решение той же задачи с начальной точкой $\theta_{i}$ и с данными Коши $U_{i 0}$. Тогда в силу предложения 4.2

$$
U_{i}(\theta) \rightarrow U(\theta)=U_{0} \quad \text { в } \mathbb{E}_{m-1, k} \text { при } i \rightarrow \infty,
$$

где $U_{i}(t)=\mathscr{D}(t) u_{i}, \quad U(t)=\mathscr{D}(t) u$. Из единственности решения $v(t, x)$ задачи $(3.12),(3.14)$, удовлетворяюшего условиям (3.17), следует, что

$$
v_{i}=\mathscr{F}_{Q_{\rho}, R_{\rho}}\left(\theta, U_{i}(\theta)\right) \text { при всех } i .
$$

Поэтому в силу (3.18) имеем

$$
E_{m-1, k,[\varkappa,-\varkappa]}\left(S(\theta)\left(v_{i}-v\right)\right) \leqslant C\left(\left\|U_{i}(\theta)-U_{0}\right\|_{m-1, k}+\left\|U_{i}(\theta)-U_{0}\right\|_{m-1, k}^{\gamma}\right) .
$$

Сопоставляя это неравенство с (3.30), получим (3.19). Предложение 3.3 полностью доказано. 


\section{§ 4. Приложение}

4.1. Срезанная нелинейность. Напомним, что функция $Q_{\rho}(\varepsilon, t, U)$ определялась по формуле (3.1).

ПРЕДЛОЖЕНИЕ 4.1. Для любых $\varepsilon \in[-1,1], \rho>0$ и челого числа $k>n / 2$ справедливы следующие утверждения.

(i) Oператор $Q_{\rho}(\varepsilon, t, U): \mathbb{R}_{t} \times \mathbb{E}_{m-1, k} \rightarrow H^{(k)}$ бесконечно дифференцируем по Фреше, причем все производные ограничены равномерно по $(\varepsilon, t, U) \in[-1,1] \times$ $\mathbb{R}_{t} \times \mathbb{E}_{m-1, k}$ для любого фиксированного $\rho>0$.

(ii) Для любого $\gamma, 0<\gamma \leqslant 1$, найдется такое $K_{1}=K_{1}(\rho, k, \gamma)>0$, что

$$
\begin{aligned}
\left\|Q_{\rho}(\varepsilon, t, U)\right\|_{(k)} & \leqslant K_{1} \min \left\{\rho,\|U\|_{m-1, k}\right\} \\
\left\|Q_{\rho}(\varepsilon, t, U)-Q_{\rho}(\varepsilon, t, V)\right\|_{(k)} & \leqslant K_{1}\left(\left\|U_{1}-V_{1}\right\|_{m-1, k}+\left\|U_{2}-V_{2}\right\|_{m-1, k}^{\gamma}\right)
\end{aligned}
$$

где $U, V \in \mathbb{E}_{m-1, k}, \quad U=U_{1}+U_{2}, \quad V=V_{1}+V_{2}$.

ДокАЗАтельство. Мы ограничимся доказательством (4.2), так как утверждения типа (i) хорошо известны в теории уравнений с частными производными (см., например, $[7$, гл. $7, \S 3])$, а неравенство (4.1) легко следует из финитности функции $Q_{\rho}$ и равномерной ограниченности ее производной.

При $\gamma=1$ неравенство (4.2) является простым следствием формулы о среднем значении. Не очевидно то, что в качестве $\gamma$ можно выбрать любое число из интервала $(0,1)$.

Неравенство (4.2) тривиально при $\min \left\{\|U\|_{m-1, k},\|V\|_{m-1, k}\right\} \geqslant \rho$. Поэтому можно считать, что $\|U\|_{m-1, k} \leqslant \rho$. Предположим сначала, что $\|V\|_{m-1, k} \leqslant 3 \rho$. Обозначим через $Q_{\rho}^{\prime}$ производную оператора $Q_{\rho}$ по $U$. Тогда в силу теоремы о среднем значении и равномерной ограниченности производной $Q_{\rho}^{\prime}$ имеем

$$
\left\|Q_{\rho}(\varepsilon, t, U)-Q_{\rho}(\varepsilon, t, V)\right\|_{(k)} \leqslant C_{1}\|U-V\|_{m-1, k} .
$$

Теперь заметим, что если $\left\|U_{2}-V_{2}\right\|_{m-1, k} \leqslant 1$, то

$$
\begin{aligned}
\|U-V\|_{m-1, k} & \leqslant\left\|U_{1}-V_{1}\right\|_{m-1, k}+\left\|U_{2}-V_{2}\right\|_{m-1, k} \\
& \leqslant\left\|U_{1}-V_{1}\right\|_{m-1, k}+\left\|U_{2}-V_{2}\right\|_{m-1, k}^{\gamma},
\end{aligned}
$$

а если $\left\|U_{2}-V_{2}\right\|_{m-1, k} \geqslant 1$, то

$$
\|U-V\|_{m-1, k} \leqslant 4 \rho \leqslant 4 \rho\left(\left\|U_{1}-V_{1}\right\|_{m-1, k}+\left\|U_{2}-V_{2}\right\|_{m-1, k}^{\gamma}\right) .
$$

Сопоставляя оценки (4.3)-(4.5), получим (4.2).

Предположим теперь, что $\|V\|_{m-1, k} \geqslant 3 \rho$. Тогда $\|U-V\|_{m-1, k} \geqslant 2 \rho$, и поэто$\mathrm{my}^{6}$

$$
\left\|U_{1}-V_{1}\right\|_{m-1, k}+\left\|U_{2}-V_{2}\right\|_{m-1, k}^{\gamma} \geqslant \rho^{\gamma} .
$$

Отсюда и из (4.1) следует, что

$$
\begin{aligned}
\left\|Q_{\rho}(\varepsilon, t, U)-Q_{\rho}(\varepsilon, t, V)\right\|_{(k)} & =\left\|Q_{\rho}(\varepsilon, t, U)\right\|_{(k)} \leqslant C_{2} \rho \\
& \leqslant C_{2} \rho^{1-\gamma}\left(\left\|U_{1}-V_{1}\right\|_{m-1, k}+\left\|U_{2}-V_{2}\right\|_{m-1, k}^{\gamma}\right) .
\end{aligned}
$$

Предложение доказано.

\footnotetext{
${ }^{6}$ Не ограничивая общности, мы считаем, что $\rho \geqslant 1$.
} 
4.2. Задача Коши для уравнения со срезанной нелинейностью. Напомним, что число $\sigma_{\max }$ определялось в (1.9).

ПРЕДЛОЖЕНИЕ 4.2. Пусть выполнены условия (P) $u(\mathrm{Q})$. Тогда для любых $\rho>0, \varkappa>\sigma_{\max }$ и челого числа $k>n / 2$ найдутся такие константы $\varepsilon_{0}>0$ и $K_{2}>0$, что при $|\varepsilon| \leqslant \varepsilon_{0}$ справедливы следующие утверждения.

(i) Для любого $\theta \in \mathbb{R}$ и произвольного набора данных Коши $\left[u_{0}, \ldots, u_{m-1}\right] \in$ $\mathbb{E}_{m-1, k}$ задача (3.2)-(3.4) имеет, притом единственное, решение и $(t, x)$. Если $u_{i}(t, x), \quad i=1,2,-$ два решения этой задачи, соответствующие данным Kouи $U_{i 0} \in \mathbb{E}_{m-1, k}$, mo

$$
E_{m-1, k}\left(u_{1}-u_{2}, t\right) \leqslant K_{2} e^{\varkappa|t-\theta|}\left\|U_{10}-U_{20}\right\|_{m-1, k} .
$$

(ii) Onератор $\mathscr{U}_{\varepsilon}^{\rho}\left(t, \theta, U_{0}\right): \mathbb{R}_{t} \times \mathbb{R}_{\theta} \times \mathbb{E}_{m-1, k} \rightarrow \mathbb{E}_{m-1, k}$, переводящий $U_{0}=$ $\left[u_{0}, \ldots, u_{m-1}\right]$ в вектор-функиию (1.3), является бесконечно дифференцируемыцм по $U_{0}$ в смыслле Фреше при любых фиксированных $t$ и $\theta$.

(iii) Оператор $\mathscr{U}_{\varepsilon}^{\rho}$ непрерывен по $\left(t, \theta, U_{0}\right) \in \mathbb{R}_{t} \times \mathbb{R}_{\theta} \times \mathbb{E}_{m-1, k}$.

ДокАЗАТЕЛЬСТво. Мы докажем только утверждение (iii), так как (i) и (ii) являются следствиями общих результатов, касающихся обыкновенных дифференциальных уравнений в банаховом пространстве.

Пусть последовательности $\left\{t_{i}\right\},\left\{\theta_{i}\right\} \subset \mathbb{R}$ и $\left\{U_{i 0}\right\} \subset \mathbb{E}_{m-1, k}$ сходятся к $t, \theta \subset \mathbb{R}$ и $U_{0} \subset \mathbb{E}_{m-1, k}$ соответственно. Обозначим через $u(t, x)$ решение задачи $(3.2)-(3.4)$, а через $U(t)$ - соответствуюшую фазовую траекторию (см. (1.3)). Тогда в силу группового свойства операторов $\mathscr{U}_{\varepsilon}^{\rho}\left(t, \theta, U_{0}\right)$ имеем

$$
\begin{aligned}
\mathscr{U}_{\varepsilon}^{\rho}\left(t_{i}, \theta_{i}, U_{i 0}\right)-\mathscr{U}_{\varepsilon}^{\rho}\left(t, \theta, U_{0}\right) & =\left(\mathscr{U}_{\varepsilon}^{\rho}\left(t_{i}, \theta_{i}, U_{i 0}\right)-\mathscr{U}_{\varepsilon}^{\rho}\left(t_{i}, \theta_{i}, U_{0}\right)\right) \\
+ & \left(\mathscr{U}_{\varepsilon}^{\rho}\left(t_{i}, \theta_{i}, U_{0}\right)-\mathscr{U}_{\varepsilon}^{\rho}\left(t_{i}, \theta_{i}, U\left(\theta_{i}\right)\right)\right)+\left(U\left(t_{i}\right)-U(t)\right) .
\end{aligned}
$$

Применяя (4.6), получим неравенство

$$
\begin{aligned}
& \left\|\mathscr{U}_{\varepsilon}^{\rho}\left(t_{i}, \theta_{i}, U_{i 0}\right)-\mathscr{U}_{\varepsilon}^{\rho}\left(t, \theta, U_{0}\right)\right\|_{m-1, k} \\
& \quad \leqslant \operatorname{const}\left(\left\|U_{i 0}-U_{0}\right\|_{m-1, k}+\left\|U(\theta)-U\left(\theta_{i}\right)\right\|_{m-1, k}\right)+\left\|U\left(t_{i}\right)-U(t)\right\|_{m-1, k},
\end{aligned}
$$

правая часть которого стремится к нулю при $i \rightarrow \infty$.

4.3. Гиперболические операторы в пространствах функций с экспоненциальным весом по $t$. Рассмотрим неоднородное уравнение

$$
P_{\varepsilon}(t, x, \partial) u \equiv \sum_{|\alpha| \leqslant m}\left(p_{\alpha}+\varepsilon q_{\alpha}(\varepsilon, t, x)\right) \partial^{\alpha} u=f(t, x) .
$$

Напомним, что пространство $\mathbb{F}_{l, k,[\mu,-\mu]}$ и норма $E_{l, k,[\mu,-\mu]}(u)$ были определены в (3.15) и (3.16). Следующее утверждение доказано в [3, теорема 2.7].

ПРЕДЛОЖЕНИЕ 4.3. Пусть выполнены условия (P), (Q) и (Н). Тогда для любого $\mu, 0 \leqslant \mu<\sigma_{\min }$, и челого числа $k \geqslant 0$ найдутся положительные константы $\varepsilon_{0}=\varepsilon_{0}(k, \mu)$ и $K_{3}=K_{3}(k, \mu)$ такие, что при $|\varepsilon| \leqslant \varepsilon_{0}$ уравнение (4.7) имеет, притом единственное, решение $u \in \mathbb{F}_{m-1, k,[\mu,-\mu]}$ для любой правой части $f \in \mathbb{F}_{0, k,[\mu,-\mu]}$. Для этого решения справедлива оценка

$$
E_{m-1, k,[\mu,-\mu]}(S(\theta) u) \leqslant K_{3} E_{0, k,[\mu,-\mu]}(S(\theta) f), \quad \theta \in \mathbb{R},
$$

где $S(\theta) w(t, x)=w(t+\theta, x)$ - оператор сдвига по $t$. 


\section{Часть II. Теорема о центральном многообразии}

\section{§ 5. Основные результаты и схема их доказательства}

5.1. Формулировка результатов. Рассматривается уравнение (0.3), в котором операторы $P$ и $Q$ удовлетворяют условиям $(\mathrm{P})$ и $(\mathrm{Q})$ из п. 1.1. Напомним, что фазовое пространство $\mathbb{E}_{m-1, k}$ уравнения (0.3) определяется как прямое произведение пространств Соболева $H^{(m-1+k-j)}, j=0, \ldots, m-1$, с нормой

$$
\|U\|_{m-1, k}=\left(\sum_{j=0}^{m-1}\left\|u_{j}\right\|_{(m-1+k-j)}^{2}\right)^{1 / 2}, \quad U=\left[u_{0}, \ldots, u_{m-1}\right],
$$

а через $\mathbb{B}_{m-1, k}(\rho)$ обозначается открытый шар в $\mathbb{E}_{m-1, k}$ радиуса $\rho>0$ с центром в нуле. Напомним также, что разрешаюший оператор задачи Коши для уравнения (0.3) был определен в п. 1.1 (см. (1.4)).

Для оператора $P(\partial)$, удовлетворяющего условию $\left(\mathrm{H}_{c}\right)$ (см. введение), через $m_{c}$ обозначается число корней полного символа $P(\tau, \xi)$, лежаших в полосе $|\operatorname{Re} \tau| \leqslant \nu$. Положим $m_{h}=m-m_{c}$. Следуюшая теорема является основным результатом второй части.

ТЕОРема 5.1. Пусть выполнены условия (P), (Q), $\left(\mathrm{H}_{c}\right)$. Пусть $\gamma \in \mathbb{R} u$ челое число $l \geqslant 1$ таковы, что

$$
0<\gamma<1, \quad l \nu+\gamma<\delta
$$

Тогда для любого челого числа $k>n / 2$ и любьх $\rho>0 u \mu \in(\nu, \delta / l)$ найдутся константы $\varepsilon_{0}>0, C>0$ и семейство непрерывных операторов ${ }^{7}$

$\mathscr{R}_{j}\left(\varepsilon ; \theta, u_{0}, \ldots, u_{m_{c}-1}\right): \mathbb{R}_{\theta} \times \mathbb{B}_{m_{c}-1, k+m_{h}}(\rho) \rightarrow H^{(m-1+k-j)}, \quad j=m_{c}, \ldots, m-1$, таких, что $\mathscr{R}_{j}(\varepsilon ; \theta, 0)=0$, и при $|\varepsilon| \leqslant \varepsilon_{0}$ справедливь следующие утверждения.

(i) Локальная инвариантность. Семейство многообразий

$$
\begin{aligned}
\mathscr{M}(\theta, \rho) & =\left\{\left[u_{0}, \ldots, u_{m-1}\right] \in \mathbb{B}_{m-1, k}(\rho): u_{j}\right. \\
& \left.=\mathscr{R}_{j}\left(\varepsilon ; \theta, u_{0}, \ldots, u_{m_{c}-1}\right), j \geqslant m_{c}\right\}
\end{aligned}
$$

согласовано с действием разрешающего оператора $\mathscr{U}_{\varepsilon}(t, \theta, \cdot)$. Другими словами, если $U_{0} \in \mathscr{M}(\theta, \rho)$ и $\mathscr{U}_{\varepsilon}\left(t, \theta, U_{0}\right) \in \mathbb{B}_{m-1, k}(\rho)$ при $t \in J$ для некоторого интервала $J \subset \mathbb{R}$, содержащего точку $\theta$, то $\mathscr{U}_{\varepsilon}\left(t, \theta, U_{0}\right) \in \mathscr{M}(t, \rho)$ при $t \in J$.

(ii)Притягиваюшее свойство. Пусть начальная точка $\theta$ и данные Коши $U_{0} \in$ $\mathbb{B}_{m-1, k}(\rho)$ такови, ито $\mathscr{U}_{\varepsilon}\left(t, \theta, U_{0}\right) \in \mathbb{B}_{m-1, k}\left(\rho_{1}\right)$ при $t \geqslant \theta$ для некоторого $\rho_{1}<\rho$. Тогда найдется $T \geqslant \theta$ и такое $V_{0} \in \mathscr{M}(T, \rho)$, что

$$
\left\|\mathscr{U}_{\varepsilon}\left(t, \theta, U_{0}\right)-\mathscr{U}_{\varepsilon}\left(t, T, V_{0}\right)\right\|_{m-1, k} \leqslant C e^{-\mu(t-\theta)}, \quad t \geqslant T .
$$

\footnotetext{
${ }^{7}$ Число $\varepsilon$ рассматривается как параметр.
} 
Аналогичное утверждение справедливо, если фазовая траектория $\mathscr{U}_{\varepsilon}\left(t, \theta, U_{0}\right)$ принадлежит $\mathbb{B}_{m-1, k}\left(\rho_{1}\right)$ на полуоси $t \leqslant \theta$. Более того, если фазовая траектория $\mathscr{D}(t) u$ некоторого решения, определенного на всей временной оси, иеликом содержится в шаре $\mathbb{B}_{m-1, k}(\rho)$, то $\mathscr{D}(t) u \in \mathscr{M}(t, \rho)$ при всех $t \in \mathbb{R}$.

(iii) Гладкость. Для любых фиксированных $\varepsilon$ и $\theta$ оператор $\mathscr{R}_{j}(\varepsilon ; \theta, \cdot)$ nринадлежит классу $C^{l, \gamma}\left(\mathbb{B}_{m_{c}-1, k+m_{h}}(\rho), H^{(m-1+k-j)}\right)$, причем норма $\left\|\mathscr{R}_{j}\right\|_{C^{l, \gamma}}$ ограничена равномерно по $(\varepsilon, \theta)$.

Перейдем теперь к описанию операторов $\mathscr{R}_{j}(\varepsilon ; \theta, \cdot)$. Для этого нам понадобится один класс символов псевдодифференциальных операторов. Подобные символы возникают при факторизации строго гиперболических полиномов, удовлетворяющих условию $\left(\mathrm{H}_{c}\right)$.

Обозначим через $S^{j}$ множество функций $p(\varepsilon, y, \xi)$, которые определены и бесконечно дифференцируемы при $(\varepsilon, y, \xi) \in[-1,1] \times \mathbb{R}_{y}^{n+1} \times i \mathbb{R}^{n}$ и удовлетворяют следующим условиям:

(i) для любых мультииндексов $\alpha, \beta$

$$
[p]_{j, \alpha, \beta}:=\sup _{(\varepsilon, y, \xi)}\left|\partial_{\xi}^{\alpha} \partial_{z}^{\beta} p(\varepsilon, y, \xi)\right|\langle\xi\rangle^{|\alpha|-j}<\infty
$$

где $\langle\xi\rangle=\left(1+\left|\xi_{1}\right|^{2}+\cdots+\left|\xi_{n}\right|^{2}\right)^{1 / 2}$;

(ii) найдется функция $p^{0}(\varepsilon, y, \xi) \in C^{\infty}\left([-1,1] \times \mathbb{R}_{y}^{n+1} \times\left(i \mathbb{R}^{n} \backslash\{0\}\right)\right)$, положительно однородная по $\xi$ порядка $j$ и такая, что

$$
\left[p-\chi p^{0}\right]_{j-1, \alpha, \beta}<\infty \text { для любых мультииндексов } \alpha, \beta,
$$

где $\chi(\xi) \in C^{\infty}\left(i \mathbb{R}^{n}\right), \chi(\xi)=0$ при $|\xi|<1$ и $\chi(\xi)=1$ при $|\xi|>2$.

Каждому символу $p(\varepsilon, y, \xi)$ ставится в соответствие псевдодифференциальный оператор

$$
p\left(\varepsilon, t, x, \partial_{x}\right) u(x)=(2 \pi)^{-n / 2} \int_{\mathbb{R}^{n}} e^{i x \zeta} p(\varepsilon, t, x, i \zeta) \hat{u}(\zeta) d \zeta
$$

где через $\hat{u}(\zeta)$ обозначается преобразование Фурье функции $u(x)$ :

$$
\hat{u}(\zeta)=(2 \pi)^{-n / 2} \int_{\mathbb{R}^{n}} e^{-i x \zeta} u(x) d x .
$$

Рассмотрим символ

$$
P_{\varepsilon}(t, x, \tau, \xi)=\sum_{|\alpha| \leqslant m}\left(p_{\alpha}+\varepsilon q_{\alpha}(\varepsilon, t, x)\right) \eta^{\alpha}, \quad \eta=(\tau, \xi) .
$$

Согласно [32, теорема 3.10] при $|\varepsilon| \ll 1$ символ (5.4) допускает факторизацию

$$
P_{\varepsilon}(y, \tau, \xi)=P_{c}(\varepsilon, y, \tau, \xi) P_{c}^{\prime}(\varepsilon, y, \tau, \xi),
$$

где $P_{c}$ и $P_{c}^{\prime}$ имеют вид

$$
\begin{aligned}
& P_{c}(\varepsilon, y, \tau, \xi)=\sum_{j=0}^{m_{c}}\left(p_{c j}(\xi)+\varepsilon q_{c j}(\varepsilon, y, \xi)\right) \tau^{m_{c}-j}, \quad p_{c j}, q_{c j} \in S^{j} \\
& P_{c}^{\prime}(\varepsilon, y, \tau, \xi)=\sum_{j=0}^{m_{h}}\left(p_{c j}^{\prime}(\xi)+\varepsilon q_{c j}^{\prime}(\varepsilon, y, \xi)\right) \tau^{m_{h}-j}, \quad p_{c j}^{\prime}, q_{c j}^{\prime} \in S^{j},
\end{aligned}
$$


причем при $\varepsilon=0$ корни символов $P_{c}$ и $P_{c}^{\prime}$ лежат соответственно в полосе $|\operatorname{Re} \tau| \leqslant \nu$ и вне полосы $|\operatorname{Re} \tau|<\delta$. В дальнейшем мы будем считать, что коэффициент при $\tau^{m_{c}}$ в символе $P_{c}$ тождественно равен единице. С помощью этого условия $P_{c}$ и $P_{c}^{\prime}$ определяются однозначно.

Обозначим через

$$
R_{j}(\varepsilon, t, x, \tau, \xi)=\sum_{i=0}^{m_{c}-1} r_{i j}(\varepsilon, t, x, \xi) \tau^{i}, \quad j=m_{c}, \ldots, m-1
$$

остаток от деления полинома $\tau^{j}$ на $P_{c}(\varepsilon, t, x, \tau, \xi)$. Легко проверить, что

$$
r_{i j}(\varepsilon, y, \xi) \in S^{j-i}, \quad i=0, \ldots, m_{c}-1
$$

и поэтому соответствующий псевдодифференциальный оператор $r_{i j}\left(\varepsilon, t, x, \partial_{x}\right)$ непрерывно отображает пространство $H^{(m-1+k-i)}$ в $H^{(m-1+k-j)}$.

ТЕОРема 5.2. Пусть выполнены условия теоремы 5.1. Тогда для каждого $\varepsilon$ найдутся непрерывные нелинейные операторы

$\mathscr{B}_{j}\left(\varepsilon ; \theta, u_{0}, \ldots, u_{m_{c}-1}\right): \mathbb{R}_{\theta} \times \mathbb{B}_{m_{c}-1, k+m_{h}}(\rho) \rightarrow H^{(m-1+k-j)}, j=m_{c}, \ldots, m-1$, такие, что $\mathscr{B}_{j}(\varepsilon ; \theta, 0)=0$, и при $|\varepsilon| \leqslant \varepsilon_{0}$ справедливо представление

$$
\begin{aligned}
& \mathscr{R}_{j}\left(\varepsilon ; \theta, u_{0}, \ldots, u_{m_{c}-1}\right)= \\
& \quad=\sum_{i=0}^{m_{c}-1} r_{i j}\left(\varepsilon, \theta, x, \partial_{x}\right) u_{i}(x)+\varepsilon \mathscr{B}_{j}\left(\varepsilon ; \theta, u_{0}, \ldots, u_{m_{c}-1}\right) .
\end{aligned}
$$

Более того, имеют место следующие утверждения.

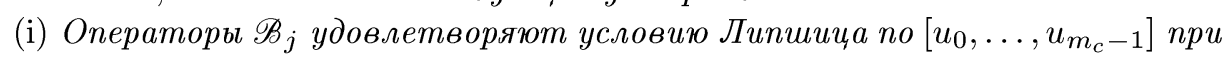
любом фиксированном $\theta \in \mathbb{R}$, причем константа Липшица ограничена равномерно по $(\varepsilon, \theta)$.

(ii) Если нелинейный член $q\left(\varepsilon, y, \partial^{m-1} u\right)($ см. (0.5)) не зависит от производных $\partial^{\alpha} u$ nри $|\alpha|=m-1$, то операторы $\mathscr{B}_{j}$ действуют непрерывно из $\mathbb{R}_{\boldsymbol{\theta}} \times \mathbb{B}_{m_{c}-1, k+m_{h}}(\rho)$ в $H^{(m+k-j)}$, и для них справедливо утвержждение (i) $c$ заменой $H^{(m-1+k-j)}$ на $H^{(m+k-j)}$.

Теоремы 5.1 и 5.2 будут доказаны в $\S 7$. Здесь мы приведем лишш наброски доказательств (см. п. 5.2).

ОПРЕДЕЛЕНИЕ. Многообразие

$$
\mathscr{M}_{\rho}=\left\{\left[\theta, u_{0}, \ldots, u_{m-1}\right] \in \mathbb{R} \times \mathbb{B}_{m-1, k}(\rho):\left[u_{0}, \ldots, u_{m-1}\right] \in \mathscr{M}(\theta, \rho)\right\},
$$

вложенное в расширенное фазовое пространство $\mathbb{R} \times \mathbb{E}_{m-1, k}$, называется иентральным многообразием уравнения (0.3).

В силу свойства локальной инвариантности (см. теорему 5.1) в окрестности любой точки центральное многообразие $\mathscr{M}_{\rho}$ состоит из интегральных кривых решений задачи (0.3), (1.2). В связи с этим возникает вопрос об описании динамики на $\mathscr{M}_{\rho}$. Следующий приниип редукиии дает частичное решение этой задачи. 
ТЕОРема 5.3. В условиях теоремы 5.1 при достаточно малых в справедливь следующие утверждения.

(i) Пусть решение $u(t, x)$ задачи (0.3), (1.2) таково, что $\mathscr{D}(t) u \in \mathscr{M}(t, \rho)$ при $t \in J$. Тогда $u(t, x)$ удовлетворяет уравнению

$$
P_{c}(\varepsilon, t, x, \partial) u-\varepsilon \mathscr{B}_{m_{c}}\left(\varepsilon ; t, u, \partial_{t} u, \ldots, \partial_{t}^{m_{c}-1} u\right)=0 .
$$

(ii) Пусть нелинейньй член $q\left(\varepsilon, t, x, \partial^{m-1} u\right)$ не зависит от производньх $\partial^{\alpha} u, \quad|\alpha|=m-1$. Предположсим, что функция $u(t, x)$, удовлетворяющая включениям

$$
\partial_{t}^{j} u \in C\left(I, H^{(m-1+k-j)}\right), \quad j=0, \ldots, m_{c}-1,
$$

для некоторого интервала $I \subset \mathbb{R}$, такова, что вектор-функция

$$
\mathscr{D}_{c}(t) u:=\left[u(t, \cdot), \partial_{t} u(t, \cdot), \ldots, \partial_{t}^{m_{c}-1} u(t, \cdot)\right]
$$

иеликом лежит в шаре $\mathbb{B}_{m_{c}-1, k+m_{h}}(\rho)$, и выполнено уравнение (5.7). Пусть $I_{1} \subset I-$ произвольный интервал, на котором кривая

$$
\left[u(t, \cdot), \ldots, \partial_{t}^{m_{c}-1} u(t, \cdot), u_{m_{c}}(t, \cdot), \ldots, u_{m-1}(t, \cdot)\right],
$$

где $u_{j}=\mathscr{R}_{j}\left(\varepsilon ; t, u, \ldots, \partial_{t}^{m_{c}-1} u\right)$ nри $j \geqslant m_{c}$, содержсится в иаре $\mathbb{B}_{m-1, k}(\rho)$. Тогда $u(t, x)$ является решением задачи $(0.3),(1.2)$ с $J=I_{1}$, причем $\mathscr{D}(t) u \in$ $\mathscr{M}(t, \rho)$ при $t \in I_{1}$.

ЗАмЕчАниЕ 5.4. В силу теоремы 5.3, если нелинейный член $q$ в (0.5) не зависит от производных порядка $m-1$, динамика на центральном многообразии описывается задачей (5.7), (5.8). Заметим, что левая часть (5.7) представляет собой малое липшицево возмушение строго гиперболического оператора $P_{c}(\varepsilon, y, \partial)$, и поэтому задача Коши для уравнения (5.7) является корректной (см., например, [19]). Вопрос о "явном" описании динамики на центральном многообразии в общем случае остается открытым. Отметим, что если $q$ зависит от старших производных, то возмущение $\varepsilon \mathscr{B}_{m_{c}}$ в уравнении (5.7) имеет порядок $m_{c}=\operatorname{ord} P_{c}$ и ввиду гиперболичности не подчинено главной линейной части.

5.2. Наброски доказательств теорем 5.1 и 5.2. В этом пункте изложены основные идеи, которые используются при построении центрального многообразия и изучении его свойств. Подробные доказательства приведены в $\S 6,7$.

Переход к уравнению со срезанной нелинейностью. Прежде всего заметим, что все утверждения теорем 5.1 и 5.2 касаются решений, фазовые траектории которых содержатся в шаре $\mathbb{B}_{m-1, k}(\rho)$. Поэтому мы можем заменить исходное уравнение (0.3) на уравнение со срезанной нелинейностью. А именно, повторяя построения из п. 3.1, для заданного $\rho>0$ и целого числа $k>n / 2$ определим функцию $Q_{\rho}(\varepsilon, t, U)$ по формуле (3.1). Вместо (0.3) будем изучать уравнение

$$
P_{\varepsilon}(t, x, \partial) u+\varepsilon Q_{\rho}(\varepsilon, t, \mathscr{D}(t) u)=0,
$$

где символ $P_{\varepsilon}(t, x, \eta)$ и вектор-функция $\mathscr{D}(t) u$ определены в $(5.4)$ и (1.3) соответственно. Мы покажем (см. теорему 7.1), что для уравнения (5.9) справедливы "глобальные варианты" утверждений теорем 5.1 и 5.2. Так как уравнения (0.3) и (5.9) совпадают на решениях, фазовые траектории которых содержатся в шаре $\mathbb{B}_{m-1, k}(\rho)$, то отсюда будут следовать теоремы 5.1 и 5.2 . 
Начальная задача $с$ условиями роста на бесконечности. Следуя стандартной схеме построения центрального многообразия (см., например, [24], [26], [33]), для заданного $\theta \in \mathbb{R}$ рассмотрим следуюшую начальную задачу для уравнения (5.9):

$$
\begin{array}{rlrl}
\partial_{t}^{j} u & \in C\left(\mathbb{R}, H^{(m-1+k-j)}\right), & & j=0, \ldots, m-1, \\
\partial_{t}^{j} u(\theta, x) & =u_{j}(x) \in H^{(m-1+k-j)}, & j=0, \ldots, m_{c}-1 .
\end{array}
$$

Таким образом, для уравнения порядка $m$ мы задали $m_{c}<m$ начальных условий. Недостающие $m_{h}=m-m_{c}$ условий заменяются ограничением на рост при $|t| \rightarrow \infty$. А именно предполагается, что энергия решения $E_{m-1, k}(u, t)$ растет не быстрее $e^{\mu|t|}$, где $\nu<\mu<\delta$. Другими словами, ищется решение, принадлежашее классу $\mathbb{F}_{m-1, k,[\mu,-\mu]}$ (см. (3.15)). Мы покажем (см. теорему 6.1), что при $|\varepsilon| \ll 1$ задача (5.9)-(5.11) имеет, притом единственное, решение $u(y) \in \mathbb{F}_{m-1, k,[\mu,-\mu]}$. Обозначим через

$$
\mathscr{G}(\varepsilon ; \theta, \cdot):\left[u_{0}, \ldots, u_{m_{c}-1}\right] \mapsto u(t, x) \quad\left(\mathbb{E}_{m_{c}-1, k+m_{h}} \rightarrow \mathbb{F}_{m-1, k,[\mu,-\mu]}\right)
$$

соответствуюший разрешаюший оператор.

Построение семейства инвариантных многообразий. Определим операторы

$$
\mathscr{R}_{j}\left(\varepsilon ; \theta, u_{0}, \ldots, u_{m_{c}-1}\right)=\left.\left(\partial_{t}^{j} \mathscr{G}\left(\varepsilon ; \theta, u_{0}, \ldots, u_{m_{c}-1}\right)\right)\right|_{t=\theta}
$$

и положим (cp. (5.2))

$$
\begin{aligned}
\mathscr{M}(\theta)=\{ & {\left[u_{0}, \ldots, u_{m-1}\right] \in \mathbb{E}_{m-1, k}: } \\
& \left.u_{j}=\mathscr{R}_{j}\left(\varepsilon ; \theta, u_{0}, \ldots, u_{m_{c}-1}\right), j \geqslant m_{c}\right\} .
\end{aligned}
$$

Таким образом, вектор-функция $U_{0}=\left[u_{0}, \ldots, u_{m-1}\right]$ принадлежит многообразию $\mathscr{M}(\theta)$ тогда и только тогда, когда она является набором данных Коши для некоторого решения $u(y) \in \mathbb{F}_{m-1, k,[\mu,-\mu]}$ уравнения (5.9). Обозначим через

$$
\mathscr{U}_{\varepsilon}^{\rho}(t, \theta, \cdot): \mathbb{E}_{m-1, k} \rightarrow \mathbb{E}_{m-1, k}, \quad t, \theta \in \mathbb{R}, \quad|\varepsilon| \ll 1,
$$

разрешаюший оператор задачи Коши для (5.9), (5.10). Из (5.14) легко следует (см. теорему 7.1), что семейство $\mathscr{M}(\theta)$ согласовано с действием оператора $\mathscr{U}_{\varepsilon}^{\rho}$, т.е. если $U_{0} \in \mathscr{M}(\theta)$ для некоторого $\theta \in \mathbb{R}$, то $\mathscr{U}_{\varepsilon}^{\rho}\left(t, \theta, U_{0}\right) \in \mathscr{M}(t)$ для любого $t \in \mathbb{R}$.

Свойство притяжения. Прежде всего заметим, что если энергия $E_{m-1, k}(u, t)$ некоторого решения $u(t, x)$ задачи $(5.9),(5.10)$ равномерно ограничена (или растет при $|t| \rightarrow \infty$ не быстрее $\left.e^{\mu|t|}\right)$, то $u \in \mathbb{F}_{m-1, k,[\mu,-\mu]}$, и поэтому соответствуюшая фазовая траектория $\mathscr{D}(t) u$ принадлежит $\mathscr{M}(t)$ для любого $t \in \mathbb{R}$. Более сложно устанавливается тот факт, что решение, энергия которого ограничена на полуоси, притягивается к некоторому решению, лежащему на многообразии (ср. (5.6))

$$
\mathscr{M}=\left\{\left[\theta, u_{0}, \ldots, u_{m-1}\right] \in \mathbb{R} \times \mathbb{E}_{m-1, k}:\left[u_{0}, \ldots, u_{m-1}\right] \in \mathscr{M}(\theta)\right\} .
$$


Набросаем доказательство этого свойства, которое основано на разрешимости неоднородного уравнения

$$
P_{\varepsilon}(t, x, \partial) u(t, x)=h(t, x)
$$

в пространстве функций с экспоненциальным весом $e^{\mu t}$ или $e^{-\mu t}$.

Пусть энергия $E_{m-1, k}(u, t)$ некоторого решения $u(y)$ задачи $(5.9),(1.2)$ с $J=$ $[\theta,+\infty)$ равномерно ограничена. Аппроксимирующее решение ишется в виде

$$
v(t, x)=\zeta(t) u(t, x)+w(t, x)
$$

где $\zeta(t) \in C^{\infty}(\mathbb{R}), \zeta(t)=0$ при $t \leqslant \theta$ и $\zeta(t)=1$ при $t \geqslant \theta+1$. Подставляя (5.16) в (5.9) и учитьвая, что $u(y)$ удовлетворяет $(5.9)$, для функции $w(t, x)$ получим уравнение

$$
P_{\varepsilon}(t, x, \partial) w(t, x)=g(t, x)-\varepsilon\left(Q_{\rho}(\varepsilon, t, V(t))-\zeta(t) Q_{\rho}(\varepsilon, t, U(t))\right)
$$

где $U(t)=\mathscr{D}(t) u, V(t)=\mathscr{D}(t) v$

$$
g(t, x)=-\sum_{l=1}^{m} \frac{1}{l !} D_{t}^{l} \zeta(t) P_{\varepsilon}^{(l)}(t, x, \partial) u(t, x), \quad P_{\varepsilon}^{(l)}(t, x, \eta)=\partial_{\tau}^{l} P_{\varepsilon}(t, x, \eta)
$$

Мы покажем (см. теорему 7.1), что уравнение (5.17) имеет, притом единственное, решение $u(y)$, принадлежащее пространству

$$
\mathbb{F}_{m-1, k,[\mu]}=\left\{f(t, x): e^{\mu t} \partial_{t}^{j} f \in C_{b}\left(\mathbb{R}, H^{(m-1+k-j)}\right), j=0, \ldots, m-1\right\}
$$

Отсюда и из (5.16) следует, что норма разности $U(t)-V(t)$ в $\mathbb{E}_{m-1, k}$ убывает как $e^{-\mu t}$ при $t \rightarrow+\infty$, а норма $V(t)$ растет при $|t| \rightarrow \infty$ не быстрее $e^{\mu|t|}$, и поэтому $V(t) \in \mathscr{M}(t)$ при всех $t \in \mathbb{R}$.

Гладкость. Мы установим гладкость $\mathscr{R}_{j}(\varepsilon ; \theta, \cdot)$ как следствие аналогичных свойств разрешаюшего оператора $\mathscr{G}(\varepsilon ; \theta, \cdot)$. Сушествование первой производной и ее гёльдеровость доказывается достаточно просто. А именно, для заданного решения $u(y) \in \mathbb{F}_{m-1, k,[\mu,-\mu]}$ задачи (5.9), (5.11) рассмотрим линеаризованное уравнение

$$
P_{\varepsilon}(t, x, \partial) v+\varepsilon\left(D Q_{\rho}\right)(\varepsilon, t, \mathscr{D}(t) u) \mathscr{D}(t) v=0,
$$

дополненное начальными условиями

$$
\partial_{t}^{j} v(\theta, x)=v_{j}(x) \in H^{(m-1+k-j)}, \quad j=0, \ldots, m_{c}-1
$$

где $\left(D Q_{\rho}\right)(\varepsilon, t, U) W$ - значение производной функции $Q_{\rho}(\varepsilon, t, U)$ по $U$ на векторе $W=\left[w_{0}, \ldots, w_{m-1}\right]$. Мы покажем (см. п. 6.3), что задача (5.19), (5.20) однозначно разрешима в пространстве $\mathbb{F}_{m-1, k,[\mu,-\mu]}$, причем решение $v^{(1)}(y)$ совпадает со значением производной оператора $\mathscr{G}(\varepsilon ; \theta, \cdot)$ на векторе $\left[v_{0}, \ldots, v_{m_{c}-1}\right]$ :

$$
v(y)=\left(D^{\mathscr{G}}\right)\left(\varepsilon ; \theta, u_{0}, \ldots, u_{m_{c}-1}\right)\left[v_{0}, \ldots, v_{m_{c}-1}\right]
$$


Сушествование производных оператора $\mathscr{G}(\varepsilon ; \theta, \cdot)$ порядка $i \geqslant 2$ доказывается несколько сложнее. Это связано с тем, что $i$-я линеаризация уравнения (5.9), вообще говоря, не разрешима в пространстве $\mathbb{F}_{m-1, k,[\mu,-\mu]}$. Например, вторая линеаризация имеет вид

$$
P_{\varepsilon}(t, x, \partial) v+\varepsilon\left(D Q_{\rho}\right)(\varepsilon, t, U(t)) V(t)=-\varepsilon\left(D^{2} Q_{\rho}\right)(\varepsilon, t, U(t))\left[V_{1}(t), V_{1}(t)\right],
$$

где $U(t)=\mathscr{D}(t) u, V(t)=\mathscr{D}(t) v, V_{1}(t)=\mathscr{D}(t) v^{(1)}$, а $\left(D^{2} Q_{\rho}\right)(\varepsilon, t, U)[W, W]$ - значение второй производной функции $Q_{\rho}(\varepsilon, t, U)$ по $U$ на векторе $W=\left[w_{0}, \ldots, w_{m-1}\right]$. Теперь заметим, что $H^{(k)}$-норма правой части уравнения (5.21) может расти при $t \rightarrow \infty$ как $e^{2 \mu|t|}$. Поэтому решение уравнения (5.21) не обязано принадлежать пространству $\mathbb{F}_{m-1, k,[\mu,-\mu]}$. Однако если число $\mu$ не очень велико (а именно выполнено неравенство $\mu<\delta / 2$, так что $\nu<\mu, 2 \mu<\delta$ ), то уравнение (5.21) имеет, притом единственное, решение $v(y) \in \mathbb{F}_{m-1, k,[2 \mu,-2 \mu]}$, удовлетворяюшее начальным условиям ${ }^{8}$

$$
\partial_{t}^{j} v(\theta, x)=0, \quad j=0, \ldots, m_{c}-1 .
$$

Мы докажем, что оператор $\mathscr{G}(\varepsilon ; \theta, \cdot)$, рассматриваемый как отображение из $\mathbb{E}_{m_{c}-1, k+m_{h}}$ в $\mathbb{F}_{m-1, k,\left[\mu^{\prime},-\mu^{\prime}\right]}$, где $2 \mu<\mu^{\prime}<\delta$, дважды непрерывно дифференцируем, и значение его второй производной на векторе $\left[v_{0}, \ldots, v_{m_{c}-1}\right]$ совпадает с решением $v(y)$ задачи $(5.21),(5.22)$.

Аналогично, $i$-я линеаризация уравнения (5.9) содержит слагаемые, растушие как $e^{i \mu|t|}$ при $t \rightarrow \infty$. При этом если $\mu<\delta / i$, а $\mathscr{G}(\varepsilon ; \theta, \cdot)$ рассматривается как оператор из $\mathbb{E}_{m_{c}-1, k+m_{h}}$ в $\mathbb{F}_{m-1, k,\left[\mu^{\prime},-\mu^{\prime}\right]}$, где $i \mu<\mu^{\prime}<\delta$, то существует $i$-я производная, значение которой на векторе $\left[v_{0}, \ldots, v_{m_{c}-1}\right]$ совпадает с решением $i$-ой линеаризации, дополненной начальными условиями (5.22) (см. $[16,33]$ и п. 6.4).

Структура операторов $\mathscr{R}_{j}(\varepsilon ; \theta, \cdot)$. Обозначим через

$$
G(\varepsilon, \theta): \mathbb{E}_{m_{c}-1, k+m_{h}} \times \mathbb{F}_{0, k,[\mu,-\mu]} \rightarrow \mathbb{F}_{m-1, k,[\mu,-\mu]}
$$

оператор, переводящий вектор-функцию $\left[u_{0}, \ldots, u_{m_{c}-1}, h\right]$ в решение

$$
u(y) \in \mathbb{F}_{m-1, k,[\mu,-\mu]}
$$

задачи $(5.15),(5.11)$. Как показано в $[3, \S 5]$, при $|\varepsilon| \ll 1$ такой оператор корректно определен и непрерывен. Теперь заметим, что

$$
\mathscr{G}\left(\varepsilon ; \theta, u_{0}, \ldots, u_{m_{c}-1}\right)=G(\varepsilon, \theta)\left[u_{0}, \ldots, u_{m_{c}-1}, 0\right]+\varepsilon G(\varepsilon, \theta)[0, \ldots, 0, f],
$$

где

$$
f(t, x)=Q_{\rho}\left(\varepsilon, t, \mathscr{D}(t) \mathscr{G}\left(\varepsilon ; \theta, u_{0}, \ldots, u_{m_{c}-1}\right)\right) .
$$

Применяя к (5.23) оператор $\partial_{t}^{j}$ и полагая $t=\theta$, получим

$$
\begin{aligned}
\mathscr{R}_{j}\left(\varepsilon ; \theta, u_{0}, \ldots, u_{m_{c}-1}\right)= & \partial_{t}^{j}\left(G(\varepsilon, \theta)\left[u_{0}, \ldots, u_{m_{c}-1}, 0\right]\right. \\
& +\varepsilon G(\varepsilon, \theta)[0, \ldots, 0, f])\left.\right|_{t=\theta} .
\end{aligned}
$$

Представление (5.5) следует из (5.25) и аналога формулы (5.5) в случае линейных уравнений (см. [3, п. 5.3]).

\footnotetext{
${ }^{8}$ Легко видеть, что если разрешающий оператор $\mathscr{G}(\varepsilon ; \theta, \cdot)$ имеет вторую производную, то ее значение на произвольной вектор-функции $\left[v_{0}, \ldots, v_{m_{c}-1}\right]$ удовлетворяет нулевым начальным условиям.
} 


\section{§ 6. Начальная задача с условиями роста на бесконечности}

Настояший параграф̆ посвяшен исследованию задачи (5.9)-(5.11). Мы докажем, что она однозначно разрешима в пространстве $\mathbb{F}_{m-1, k,[\mu,-\mu]}$, причем разрешающий оператор является гладким в соответствующих пространствах. Полученные результаты будут использоваться в $\S 7$ при доказательстве теорем 5.1 и 5.2 .

6.1. Формулировка результатов. Напомним, что пространство $\mathbb{F}_{l, k,[\mu,-\mu]}$ и соответствуюшая норма $E_{l, k,[\mu,-\mu]}(\cdot)$ определены в $(3.15)$ и (3.16). Для $\theta \in \mathbb{R}$ и функции $w(t, x)$ положим $S(\theta) w(t, x)=w(t+\theta, x)$.

ТЕОРЕМа 6.1. Пусть выполнены условия (P), (Q) и $\left(\mathrm{H}_{c}\right)$. Тогда для любьх $\rho>0, \mu \in(\nu, \delta)$ и целого числа $k>n / 2$ найдется такая константа $\varepsilon_{0}>0$, что при $|\varepsilon| \leqslant \varepsilon_{0}$ справедливь следующие утверәсдения.

(i) Для любого $\theta \in \mathbb{R}$ и начальных данньх $\left[u_{0}, \ldots, u_{m_{c}-1}\right] \in \mathbb{E}_{m_{c}-1, k+m_{h}}$ задача (5.9)-(5.11) имеет, притом единственное, решение

$$
u(y) \in \mathbb{F}_{m-1, k,[\mu,-\mu]} .
$$

(ii) Если $u_{1}, u_{2} \in \mathbb{F}_{m-1, k,[\mu,-\mu]}-$ два решения, соответствующие начальным точкам $\theta_{1}, \theta_{2} \in \mathbb{R},\left|\theta_{1}-\theta_{2}\right| \leqslant 1$, и начальным данным $U_{1}, U_{2} \in$ $\mathbb{E}_{m_{c}-1, k+m_{h}}, m o$

$$
E_{m-1, k,[\mu,-\mu]}\left(S\left(\theta_{1}\right)\left(u_{1}-u_{2}\right)\right) \leqslant C\left\|U_{1}-U_{2}\right\|_{m_{c}-1, k+m_{h}}+b\left(\theta_{1}, U_{1} ;\left|\theta_{1}-\theta_{2}\right|\right)
$$

где константа $C>0$ и непрерывная функиия $b\left(\theta_{1}, U_{1} ; r\right) \geqslant 0$ переменной $r \in[0,1]$ зависят от параметров $k, \rho u \mu$, причем $b\left(\theta_{1}, U_{1} ; r\right)$ стремится $\kappa$ нулю при $r \rightarrow 0$.

Теорема 6.1 будет доказана в п. 6.2.

Напомним, что оператор $\mathscr{G}\left(\varepsilon ; \theta, u_{0}, \ldots, u_{m_{c}-1}\right)$ определен в п. 5.2 (см. формулу (5.12)). Из неравенства (6.1), в частности, следует, что он непрерывен по совокупности переменных $\left(\theta, u_{0}, \ldots, u_{m_{c}-1}\right)$. В следующей теореме доказывается, что при фиксированном $\theta$ и достаточно малых $\varepsilon$ оператор $\mathscr{G}(\varepsilon ; \theta, \cdot)$ является гладким в соответствующих пространствах.

ТеОРема 6.2. Пусть выполнены условия $(\mathrm{P}),(\mathrm{Q}),\left(\mathrm{H}_{c}\right)$ и неравенства (5.1), где $l \geqslant 1$ - иелое число. Тогда найдется такое $\varepsilon_{0}>0$, что при $|\varepsilon| \leqslant$ $\varepsilon_{0}$ оператор $\mathscr{G}(\varepsilon ; \theta, \cdot)$ принадлежсит классу $C^{l, \gamma}\left(\mathbb{E}_{m_{c}-1, k+m_{h}}, \mathbb{F}_{m-1, k,\left[\mu^{\prime},-\mu^{\prime}\right]}\right)$, где $\mu^{\prime}=l \mu+\gamma$, причем полунорма $|\mathscr{G}|_{C^{l, \gamma}}$ ограничена равномерно по $(\varepsilon, \theta)$.

Доказательство теоремы 6.2 приведено в пп. 6.3 и 6.4.

6.2. Доказательство теоремы 6.1. Нам понадобится следующее утверждение о разрешимости линейных уравнений в пространстве $\mathbb{F}_{m-1, k,[\mu,-\mu]}$. Его доказательство можно найти в $[3, \S 4]$. 
ПРЕДЛОЖЕНИЕ 6.3. В условиях теоремы 6.1 для любого $\mu \in(\nu, \delta)$ и иелого числа $k \geqslant 0$ найдутся такие константы $\varepsilon_{0}>0 u C>0$, что при $|\varepsilon| \leqslant \varepsilon_{0}$ задача (5.15), (5.11) с правой частью $h \in \mathbb{F}_{0, k,[\mu,-\mu]}$ и начальными даннымми $\left[u_{0}, \ldots, u_{m_{c}-1}\right] \in \mathbb{E}_{m_{c}-1, k+m_{h}}$ имеет, притом единственное, решение $u(y) \in$ $\mathbb{F}_{m-1, k,[\mu,-\mu]}$, которое удовлетворяет неравенству

$$
E_{m-1, k,[\mu,-\mu]}(S(\theta) u) \leqslant C\left(\sum_{j=0}^{m_{c}-1}\left\|u_{j}\right\|_{(m-1+k-j)}+E_{0, k,[\mu,-\mu]}(S(\theta) h)\right) .
$$

ДокАЗАТЕЛЬСТво. (i) Для простоты обозначений будем считать, что $\theta=0$. Сушествование решения доказывается методом сжимающих отображений. Напомним, что для функции $w(t, x)$ через $\mathscr{D}(t) w$ обозначается соответствующая фазовая траектория (см. (1.3)). Рассмотрим оператор $A$, переводящий функцию $v(y) \in$ $\mathbb{F}_{m-1, k,[\mu,-\mu]}$ в решение $u(y) \in \mathbb{F}_{m-1, k,[\mu,-\mu]}$ уравнения

$$
P_{\varepsilon}(t, x, \partial) u(t, x)=-\varepsilon Q_{\rho}(\varepsilon, t, \mathscr{D}(t) v)
$$

с начальными условиями (5.11). Очевидно, что неподвижная точка оператора $A$ является искомым решением.

В силу леммы 8.2 правая часть (6.3) принадлежит $\mathbb{F}_{0, k,[\mu,-\mu]}$. Поэтому согласно предложению 6.3 при $|\varepsilon| \ll 1$ задача (6.3), (5.11) имеет, притом единственное, решение $u \in \mathbb{F}_{m-1, k,[\mu,-\mu]}$. Таким образом, оператор

$$
A: \mathbb{F}_{m-1, k,[\mu,-\mu]} \rightarrow \mathbb{F}_{m-1, k,[\mu,-\mu]}
$$

корректно определен. Более того, если $u_{i}=A\left(v_{i}\right)$, то разность $u=u_{1}-u_{2}$ удовлетворяет уравнению (5.15) с правой частью

$$
h(t, x)=\varepsilon\left(Q_{\rho}\left(\varepsilon, t, \mathscr{D}(t) v_{2}\right)-Q_{\rho}\left(\varepsilon, t, \mathscr{D}(t) v_{1}\right)\right)
$$

и имеет нулевые данные Коши до порядка $m_{c}-1$. Поэтому в силу неравенства (6.2) имеем

$$
E_{m-1, k,[\mu,-\mu]}(u) \leqslant C E_{0, k,[\mu,-\mu]}(h) .
$$

Согласно лемме 8.2 имеет место неравенство

$$
E_{0, k,[\mu,-\mu]}(h) \leqslant C_{1}|\varepsilon| E_{m-1, k,[\mu,-\mu]}\left(v_{1}-v_{2}\right) .
$$

Подставляя эту оценку в (6.4), получим

$$
E_{m-1, k,[\mu,-\mu]}\left(u_{1}-u_{2}\right) \leqslant C_{2}|\varepsilon| E_{m-1, k,[\mu,-\mu]}\left(v_{1}-v_{2}\right) .
$$

Отсюда следует, что при $|\varepsilon| \ll 1$ оператор $A$ является сжимаюшим и поэтому имеет неподвижную точку $u(y)$.

Единственность построенного решения следует из неравенства (6.1), которое устанавливается ниже. 
(ii) Разность $u=u_{1}-u_{2}$ является решением уравнения (5.15) с правой частью

$$
h(t, x)=\varepsilon\left(Q_{\rho}\left(\varepsilon, t, \mathscr{D}(t) u_{2}\right)-Q_{\rho}\left(\varepsilon, t, \mathscr{D}(t) u_{1}\right)\right)
$$

Поэтому в силу неравенства (6.2) имеем

$$
\begin{aligned}
& E_{m-1, k,[\mu,-\mu]}\left(S\left(\theta_{2}\right) u\right) \\
& \quad \leqslant C\left(\sum_{j=0}^{m_{c}-1}\left\|\partial_{t}^{j} u\left(\theta_{2}, \cdot\right)\right\|_{(m-1+k-j)}+E_{0, k,[\mu,-\mu]}\left(S\left(\theta_{2}\right) h\right)\right) .
\end{aligned}
$$

Оценим правую часть (6.6). Положим

$$
u_{i j}(x)=\partial_{t}^{j} u_{i}\left(\theta_{i}, x\right), \quad i=1,2, \quad j=0, \ldots, m_{c}-1
$$

Тогда

$$
\partial_{t}^{j} u\left(\theta_{2}, x\right)=\left(\partial_{t}^{j} u_{1}\left(\theta_{2}, x\right)-u_{1 j}(x)\right)+\left(u_{1 j}(x)-u_{2 j}(x)\right),
$$

откуда следует, что

$$
\left\|\partial_{t}^{j} u\left(\theta_{2}, \cdot\right)\right\|_{(m-1+k-j)} \leqslant b_{1}\left(\theta_{1}, U_{1} ;\left|\theta_{1}-\theta_{2}\right|\right)+\left\|u_{1 j}-u_{2 j}\right\|_{(m-1+k-j)},
$$

где непрерывная по $r \geqslant 0$ функция $b_{1}\left(\theta_{1}, U_{1} ; r\right) \geqslant 0$ стремится к нулю при $r \rightarrow 0$. Далее, в силу леммы 8.2 для (6.5) справедлива оценка

$$
E_{0, k,[\mu,-\mu]}\left(S\left(\theta_{2}\right) h\right) \leqslant C_{1}|\varepsilon| E_{m-1, k,[\mu,-\mu]}\left(S\left(\theta_{2}\right) u\right)
$$

Подставляя (6.7) и (6.8) в (6.6), при $|\varepsilon| \ll 1$ получим

$E_{m-1, k,[\mu,-\mu]}\left(S\left(\theta_{2}\right) u\right) \leqslant C\left(\sum_{j=0}^{m_{c}-1}\left\|u_{1 j}-u_{2 j}\right\|_{(m-1+k-j)}+b_{1}\left(\theta_{1}, U_{1} ;\left|\theta_{1}-\theta_{2}\right|\right)\right)$.

Остается заметить, что при $\theta \in \mathbb{R}$ нормы $E_{m-1, k,[\mu,-\mu]}(S(\theta) u)$ эквивалентны, причем константы эквивалентности ограничены, если $\theta$ меняется на конечном интервале.

6.3. Доказательство теоремы 6.2 в случае $l=1$. Прежде всего заметим, что для любой функции $u(t, x)$, удовлетворяющей включениям $(5.10)$, и любых начальных данных $\left[v_{0}, \ldots, v_{m_{c}-1}\right] \in \mathbb{E}_{m_{c}-1, k+m_{h}}$ задача $(5.19),(5.20)$ имеет, притом единственное, решение $v(y) \in \mathbb{F}_{m-1, k,[\mu,-\mu]}$. Это утверждение легко доказывается с помощью рассуждений из п. 6.2. Обозначим через

$$
\mathscr{G}_{1}\left(\varepsilon ; \theta, u_{0}, \ldots, u_{m_{c}-1}\right): \mathbb{E}_{m_{c}-1, k+m_{h}} \rightarrow \mathbb{F}_{m-1, k,[\mu,-\mu]}
$$

линейный ограниченный оператор, переводящий вектор $\left[v_{0}, \ldots, v_{m_{c}-1}\right]$ в решение $v \in \mathbb{F}_{m-1, k,[\mu,-\mu]}$ задачи $(5.19),(5.20)$, в которой $u=\mathscr{G}\left(\varepsilon ; \theta, u_{0}, \ldots, u_{m_{c}-1}\right)$. В дальнейшем для простоты будем считать, что $\theta=0$, причем в обозначениях операторов $\mathscr{G}_{\text {и }} \mathscr{G}_{1}$ параметр $\theta$ будем опускать. 
В силу обратной теоремы Тейлора (см. [13] или [11, п. 1.2.5]) сушествование производной оператора

$$
\mathscr{G}(\varepsilon ; \cdot): \mathbb{E}_{m_{c}-1, k+m_{h}} \rightarrow \mathbb{F}_{m-1, k,\left[\mu^{\prime},-\mu^{\prime}\right]}, \quad \mu^{\prime}=\mu+\gamma,
$$

ее гёльдеровость и равномерная ограниченность полунормы $|\mathscr{G}|_{C^{1, \gamma}}$ будут доказаны, если мы покажем, что

$$
\mathscr{G}\left(\varepsilon ; U_{0}+V_{0}\right)=\mathscr{G}\left(\varepsilon ; U_{0}\right)+\mathscr{G}_{1}\left(\varepsilon ; U_{0}\right) V_{0}+\mathscr{F}_{1}\left(\varepsilon ; U_{0}, V_{0}\right),
$$

где $U_{0}=\left[u_{0}, \ldots, u_{m_{c}-1}\right], V_{0}=\left[v_{0}, \ldots, v_{m_{c}-1}\right]$, причем оператор $\mathscr{F}_{1}$ допускает оценку

$$
E_{0, k,\left[\mu^{\prime},-\mu^{\prime}\right]}\left(\mathscr{F}_{1}\left(\varepsilon ; U_{0}, V_{0}\right)\right) \leqslant \text { const }\left\|V_{0}\right\|_{m_{c}-1, k+m_{h}}^{1+\gamma} .
$$

Положим

$$
\tilde{u}(y)=\mathscr{G}\left(\varepsilon ; U_{0}+V_{0}\right), \quad u(y)=\mathscr{G}\left(\varepsilon ; U_{0}\right), \quad v^{(1)}(y)=\mathscr{G}_{1}\left(\varepsilon ; U_{0}\right) V_{0} .
$$

Непосредственная проверка показывает, что функция $w:=\tilde{u}-u-v^{(1)}=\mathscr{F}_{1}\left(\varepsilon ; U_{0}\right.$, $\left.V_{0}\right)$ является решением задачи

$$
\begin{gathered}
P_{\varepsilon}(t, x, \partial) w(t, x)=-\varepsilon h(t, x), \\
\partial_{t}^{j} w(0, x)=0, \quad j=0, \ldots, m_{c}-1,
\end{gathered}
$$

где

$$
\begin{aligned}
h(t, x) & =h_{1}(t, x)+h_{2}(t, x), \\
h_{1}(t, x) & =Q_{\rho}(\varepsilon, t, \mathscr{D}(t) \tilde{u})-Q_{\rho}(\varepsilon, t, \mathscr{D}(t) u)-\left(D Q_{\rho}\right)(\varepsilon, t, \mathscr{D}(t) u) \mathscr{D}(t)(\tilde{u}-u), \\
h_{2}(t, x) & =\left(D Q_{\rho}\right)(\varepsilon, t, \mathscr{D}(t) u) \mathscr{D}(t) w .
\end{aligned}
$$

В силу неравенства (6.2), в котором $\theta=0$ и $\mu=\mu^{\prime}$, имеем

$$
E_{m-1, k,\left[\mu^{\prime},-\mu^{\prime}\right]}(w) \leqslant C|\varepsilon| E_{0, k,\left[\mu^{\prime},-\mu^{\prime}\right]}(h) .
$$

Оценим правую часть (6.13). Согласно лемме 8.1 для $h_{1}$ справедлива оценка

$$
\left\|h_{1}(t, \cdot)\right\|_{(k)} \leqslant C_{1}\|\mathscr{D}(t)(\tilde{u}-u)\|_{m-1, k}^{1+\gamma} .
$$

Отсюда и из неравенства (6.1) с $\theta_{1}=\theta_{2}=0$ следует, что

$$
E_{0, k,\left[\mu^{\prime},-\mu^{\prime}\right]}\left(h_{1}\right) \leqslant C_{2} \sum_{j=0}^{m_{c}-1}\left\|v_{j}\right\|_{(m-1+k-j)}^{1+\gamma} .
$$

Далее, в силу равномерной ограниченности производных функции $Q_{\rho}$ имеем

$$
E_{0, k,\left[\mu^{\prime},-\mu^{\prime}\right]}\left(h_{2}\right) \leqslant C_{3} E_{m-1, k,\left[\mu^{\prime},-\mu^{\prime}\right]}(w) .
$$

Подставляя (6.14) и (6.15) в неравенство (6.13), в котором $h=h_{1}+h_{2}$, получим

$$
E_{m-1, k,\left[\mu^{\prime},-\mu^{\prime}\right]}(w) \leqslant C_{4} \sum_{j=0}^{m_{c}-1}\left\|v_{j}\right\|_{(m-1+k-j)}^{1+\gamma},
$$

что равносильно неравенству (6.10). 
6.4. Доказательство теоремы 6.2 в случае $l \geqslant 2$. Несмотря на название пункта, мы ограничимся случаем $l=2$. Доказательство теоремы для произвольного $l \geqslant 3$ технически довольно сложно, однако не содержит принципиально новых идей. Подробное доказательство аналогичного утверждения в случае обыкновенных дифференциальных уравнений и абстрактных уравнений в банаховом пространстве можно найти в [16], [33].

Как и в п. 6.3 , будем считать, что $\theta=0$. Мы хотим показать, что оператор

$$
\mathscr{G}(\varepsilon ; \cdot): \mathbb{E}_{m_{c}-1, k+m_{h}} \rightarrow \mathbb{F}_{m-1, k,\left[\mu^{\prime},-\mu^{\prime}\right]}, \quad \mu^{\prime}=2 \mu+\gamma,
$$

дважды непрерывно дифференцируем по Фреше, причем вторая производная удовлетворяет условию Гёльдера с показателем $\gamma$, а полунорма $|\mathscr{G}|_{C^{2, \gamma}}$ равномерно ограничена.

Прежде всего заметим, что для любой функции $u(t, x)$, удовлетворяющей включениям (5.10), задача $(5.21),(5.22)$ (в которой $U(t)=\mathscr{D}(t) u, V(t)=\mathscr{D}(t) v$, $\left.V_{1}(t)=\mathscr{D}(t) v^{(1)}\right)$ имеет, притом единственное, решение $v(y) \in \mathbb{F}_{m-1, k,[2 \mu,-2 \mu]}(\mathrm{cp}$. п. 6.3). Обозначим через

$$
\mathscr{G}_{2}\left(\varepsilon ; u_{0}, \ldots, u_{m_{c}-1}\right): \mathbb{E}_{m_{c}-1, k+m_{h}} \rightarrow \mathbb{F}_{m-1, k,[2 \mu,-2 \mu]}
$$

ограниченный квадратичный оператор, переводяший $\left[v_{0}, \ldots, v_{m_{c}-1}\right]$ в решение $v \in$ $\mathbb{F}_{m-1, k,[2 \mu,-2 \mu]}$ задачи $(5.21),(5.22)$, в которой $u=\mathscr{G}\left(\varepsilon ; u_{0}, \ldots, u_{m_{c}-1}\right)$.

В силу обратной теоремы Тейлора нам надо проверить, что

$$
\mathscr{G}\left(\varepsilon ; U_{0}+V_{0}\right)=\mathscr{G}\left(\varepsilon ; U_{0}\right)+\mathscr{G}_{1}\left(\varepsilon ; U_{0}\right) V_{0}+\frac{1}{2} \mathscr{G}_{2}\left(\varepsilon ; U_{0}\right)\left[V_{0}, V_{0}\right]+\mathscr{F}_{2}\left(\varepsilon ; U_{0}, V_{0}\right)
$$

где $U_{0}=\left[u_{0}, \ldots, u_{m_{c}-1}\right], V_{0}=\left[v_{0}, \ldots, v_{m_{c}-1}\right]$, а оператор $\mathscr{F}_{2}$ допускает оценку

$$
E_{0, k,\left[\mu^{\prime},-\mu^{\prime}\right]}\left(\mathscr{F}_{2}\left(\varepsilon ; U_{0}, V_{0}\right)\right) \leqslant \text { const }\left\|V_{0}\right\|_{m_{c}-1, k+m_{h}}^{2+\gamma} .
$$

Положим $v^{(2)}(y)=\mathscr{G}_{2}\left(\varepsilon ; U_{0}\right)\left[V_{0}, V_{0}\right]$. Тогда функция

$$
w:=\tilde{u}-u-v^{(1)}-\frac{1}{2} v^{(2)}=\mathscr{F}_{2}\left(\varepsilon ; U_{0}, V_{0}\right)
$$

является решением задачи (6.11), (6.12), где ${ }^{9} h=h_{1}+h_{2}+h_{3}$,

$$
\begin{aligned}
h_{1}(t, x)= & Q_{\rho}(\varepsilon, t, \tilde{u})-Q_{\rho}(\varepsilon, t, u)-\left(D Q_{\rho}\right)(\varepsilon, t, u)(\tilde{u}-u) \\
& -\frac{1}{2}\left(D^{2} Q_{\rho}\right)(\varepsilon, t, u)[\tilde{u}-u, \tilde{u}-u], \\
h_{2}(t, x)= & \left(D Q_{\rho}\right)(\varepsilon, t, u) w, \\
h_{3}(t, x)= & \frac{1}{2}\left(\left(D^{2} Q_{\rho}\right)(\varepsilon, t, u)[\tilde{u}-u, \tilde{u}-u]-\left(D^{2} Q_{\rho}\right)(\varepsilon, t, u)\left[v^{(1)}, v^{(1)}\right]\right) .
\end{aligned}
$$

\footnotetext{
${ }^{9}$ Для простоты формул до конца настоящего параграфа оператор $\mathscr{D}(t)$ будем опускать. Из контекста всегда будет ясно, где он должен присутствовать.
} 
Поэтому в силу (6.2) для $w(y)$ справедливо неравенство (6.13) с $\mu^{\prime}=2 \mu+\gamma$. Оценим правую часть (6.13). Пользуясь леммой 8.1 и неравенством (6.1), легко показать, что (ср. вывод (6.14))

$$
E_{0, k,\left[\mu^{\prime},-\mu^{\prime}\right]}\left(h_{1}\right) \leqslant C_{1} \sum_{j=0}^{m_{c}-1}\left\|v_{j}\right\|_{(m-1+k-j)}^{2+\gamma} .
$$

Функция $h_{2}(t, x)$ удовлетворяет неравенству (6.15) с $\mu^{\prime}=2 \mu+\gamma$. Для оценки нормы $h_{3}$ заметим, что

$$
h_{3}(t, x)=\frac{1}{2}\left(D^{2} Q_{\rho}\right)(\varepsilon, t, u)\left[\tilde{u}-u-v^{(1)}, \tilde{u}-u+v^{(1)}\right] .
$$

Сопоставляя это соотношение с (6.9) и (6.10), получим, что для $h_{3}$ справедлива оценка вида (6.17). Отсюда и из (6.13) следует требуемое неравенство (6.16). Подробности мы оставляем читателю.

\section{§ 7. Доказательство основных результатов}

Настоящий параграф посвящен доказательству теорем 5.1 - 5.3. Мы сначала покажем (см. теорему 7.1), что для уравнения со срезанной нелинейностью (5.9) справедливы глобальные варианты утверждений теорем 5.1 и 5.2. В качестве простого следствия мы получим теоремы 5.1 и 5.2. Затем, пользуясь установленными результатами и единственностью решения задачи Коши для строго гиперболических уравнений с липшицевой нелинейностью, мы докажем теорему 5.3.

7.1. Центральное многообразие для уравнения со срезанной нелинейностью. Напомним, что через $\mathscr{U}_{\varepsilon}^{\rho}\left(t, \theta, U_{0}\right)$ обозначается разрешающий оператор задачи Коши (5.9), (5.10), (1.1). В силу предложения 4.2 при $|\varepsilon| \ll 1$ этот оператор определен для всех $t, \theta \in \mathbb{R}$.

ТЕОРема 7.1. Пусть выполнены условия (P), (Q), $\left(\mathrm{H}_{c}\right)$ и неравенства (5.1), где $l \geqslant 1$ - иелое число. Тогда для любых $\rho>0, \mu \in(\nu, \delta / l)$ и челого числа $k>n / 2$ найдется константа $\varepsilon_{0}>0$ и семейство непрерывных операторов

$$
\begin{gathered}
\mathscr{R}_{j}\left(\varepsilon ; \theta, u_{0}, \ldots, u_{m_{c}-1}\right): \mathbb{R}_{\theta} \times \mathbb{E}_{m_{c}-1, k+m_{h}} \rightarrow H^{(m-1+k-j)}, \\
j=m_{c}, \ldots, m-1,
\end{gathered}
$$

таких, что $\mathscr{R}_{j}(\varepsilon ; \theta, 0)=0$, и при $|\varepsilon| \leqslant \varepsilon_{0}$ справедливь следующие утверждения.

(i) Семейство многообразий $\mathscr{M}(\theta)$, определенных по формуле (5.14), согласовано с действием разрешающего оператора $\mathscr{U}_{\varepsilon}^{\rho}(t, \theta, \cdot)$ в том смысле, что если $U_{0} \in \mathscr{M}(\theta)$ для некоторого $\theta \in \mathbb{R}$, то $\mathscr{U}_{\varepsilon}^{\rho}\left(t, \theta, U_{0}\right) \in \mathscr{M}(t)$ при всех $t \in \mathbb{R}$.

(ii) Если функция $u(t, x) \in \mathbb{F}_{m-1, k,[\mu,-\mu]}$ удовлетворяет уравненил (5.9), mо $\mathscr{D}(t) u \in \mathscr{M}(t)$ nри всех $t \in \mathbb{R}$.

(iii) Eсли энергия $E_{m-1, k}(u, t)$ некоторого решения $u(t, x)$ задачи (5.9), (5.10) растет при $t \rightarrow+\infty$ не быстрее $e^{\mu t}$, то найдется такое решение $v(t, x) \in$ $\mathbb{F}_{m-1, k,[\mu,-\mu]}$ уравнения (5.9), что $\mathscr{D}(t) v \in \mathscr{M}(t)$ при всех $t \in \mathbb{R} u$

$$
E_{m-1, k}(u-v, t) \leqslant C e^{-\mu(t-\theta)} E_{m-1, k}(u, \theta), \quad t \geqslant \theta,
$$


где константа $C>0$ не зависит от $t, \theta$ и и(у). Аналогичное утверждение справедливо для решений, әнергия которых растет при $t \rightarrow-\infty$ не быстрее $e^{-\mu t}$.

(iv) Для любых фиксированных $\varepsilon$ u $\theta$ оператор $\mathscr{R}_{j}(\varepsilon ; \theta, \cdot)$ принадлежит классу $C^{l, \gamma}\left(\mathbb{E}_{m_{c}-1, k+m_{h}}, H^{(m-1+k-j)}\right)$, причем полунорма $\left|\mathscr{R}_{j}\right|_{C^{l, \gamma}}$ ограничена равномерно по $(\varepsilon, \theta)$.

(v) Найдутся непрерывные нелинейные операторы

$$
\begin{aligned}
\mathscr{B}_{j}\left(\varepsilon ; \theta, u_{0}, \ldots, u_{m_{c}-1}\right) & : \mathbb{R}_{\theta} \times \mathbb{E}_{m_{c}-1, k+m_{h}} \rightarrow H^{(m-1+k-j)}, \\
j & =m_{c}, \ldots, m-1,
\end{aligned}
$$

такие, что $\mathscr{B}_{j}(\varepsilon ; \theta, 0)=0$, и имеет место представление (5.5). Более того, при фиксированном $\theta \in \mathbb{R}$ операторы $\mathscr{B}_{j}$ удовлетворяют глобальному условию Липшица:

$$
\left\|\mathscr{B}_{j}\left(\varepsilon ; \theta, U_{0}\right)-\mathscr{B}_{j}\left(\varepsilon ; \theta, V_{0}\right)\right\|_{(m-1+k-j)} \leqslant \text { const }\left\|U_{0}-V_{0}\right\|_{m_{c}-1, k+m_{h}},
$$

где $U_{0}, V_{0} \in \mathbb{E}_{m_{c}-1, k+m_{h}}$.

(vi) Если нелинейный член $q\left(\varepsilon, t, x, \partial^{m-1} u\right)$ в формуле (0.5) не зависит от производных порядка $m-1$, то справедливо утверждение (v), в котором пространство $H^{(m-1+k-j)}\left(c \mathcal{M} .(7.3)\right.$ и (7.4)) заменено на $H^{(m+k-j)}$.

ДокаЗАТЕльство. Определим $\mathscr{R}_{j}$ по формуле $(5.13)$, где $\mathscr{G}\left(\varepsilon ; \theta, U_{0}\right)$ - разрешающий оператор задачи (5.9)-(5.11) (см. (5.12)). Тогда в силу единственности имеем $\mathscr{G}(\varepsilon ; \theta, 0)=0$ и, следовательно, $\mathscr{R}_{j}(\varepsilon ; \theta, 0)=0$. Более того, из неравенства (6.1) вытекает, что $\mathscr{G}$ непрерывен по совокупности переменных $\left(\theta, U_{0}\right)$, и поэтому оператор $\mathscr{R}_{j}$ также обладает этим свойством. Докажем теперь утверждения (i) - (vi).

(i) Предположим, что $U_{0} \in \mathscr{M}(\theta)$ для некоторого $\theta \in \mathbb{R}$, и положим $u=$ $\mathscr{G}\left(\varepsilon ; \theta, U_{0}\right)$. Нам надо показать, что

$$
\partial_{t}^{j} u(t, \cdot)=\mathscr{R}_{j}\left(\varepsilon ; t, \mathscr{D}_{c}(t) u\right) \text { при всех } t \in \mathbb{R} .
$$

Легко видеть, что (7.5) вытекает из соотношения

$$
u(t, x)=\mathscr{G}\left(\varepsilon ; t, \mathscr{D}_{c}(t) u\right), \quad t \in \mathbb{R},
$$

которое является следствием единственности решения задачи (5.9)-(5.11).

(ii) Пусть функция $u \in \mathbb{F}_{m-1, k,[\mu,-\mu]}$ является решением уравнения (5.9). Тогда вьполнено (7.6), откуда следует требуемое утверждение.

(iii) Предположим, что для некоторого решения $u(y)$ задачи $(5.9),(5.10)$ выполнено неравенство

$$
E_{m-1, k}(u, t) \leqslant \operatorname{const} e^{\mu t}, \quad t \geqslant 0 .
$$

Аппроксимируюшее решение $v(y)$ ищется в виде (5.16). После подстановки $v(y)$ в (5.9) для неизвестной функции $w(y)$ получим уравнение $(5.17)$, в котором $U(t)=$ $\mathscr{D}(t) u, V(t)=\mathscr{D}(t) v$, а функция $g(t, x)$ имеет вид (5.18). Покажем, что это уравнение однозначно разрешимо в пространстве $\mathbb{F}_{m-1, k,[\mu]}$, причем решение $w(y)$ удовлетворяет неравенству

$$
E_{m-1, k,[\mu]}(w):=\sup _{t \in \mathbb{R}} e^{\mu t} E_{m-1, k}(w, t) \leqslant \operatorname{const} E_{m-1, k}(u, \theta) .
$$

Для этого нам понадобится следующее вспомогательное утверждение, которое является следствием теоремы 2.6 из [3]. 
ПРЕДЛОЖЕНИЕ 7.2. В условиях теоремы 7.1 для любого $\mu \in(\nu, \delta)$ и иелого числа $k \geqslant 0$ найдутся такие константы $\varepsilon_{0}>0$ и $C>0$, что при $|\varepsilon| \leqslant \varepsilon_{0}$ уравнение (5.15) с правой частью $h \in \mathbb{F}_{0, k,[\mu]}$ имеет, притом единственное, решение $и(y) \in \mathbb{F}_{m-1, k,[\mu]}$. Это решение удовлетворяет неравенству

$$
E_{m-1, k,[\mu]}(S(\theta) u) \leqslant E_{0, k,[\mu]}(S(\theta) h) \quad \text { для любого } \quad \theta \in \mathbb{R} .
$$

Рассмотрим оператор $A$, переводяший функцию $z \in \mathbb{F}_{m-1, k,[\mu]}$ в решение $w \in$ $\mathbb{F}_{m-1, k,[\mu]}$ уравнения

$$
P_{\varepsilon}(t, x, \partial) w(t, x)=g(t, x)-\varepsilon g_{1}(t, x),
$$

где

$$
g_{1}(t, x)=Q_{\rho}(\varepsilon, t, \mathscr{D}(t)(\zeta u+z))-\zeta(t) Q_{\rho}(\varepsilon, t, \mathscr{D}(t) u) .
$$

Мы покажем, что при $|\varepsilon| \ll 1$ оператор $A$ корректно определен и является сжимающим в пространстве $\mathbb{F}_{m-1, k,[\mu]}$. Отсюда будет следовать, что $A$ имеет неподвижную точку $w(y)$, которая и есть искомое решение.

Прежде всего заметим, что для любой функции $u(t, x)$, удовлетворяющей включениям (5.10), правая часть уравнения (7.9) принадлежит пространству $\mathbb{F}_{0, k,[\mu]}$ и удовлетворяет неравенству

$$
E_{0, k,[\mu]}\left(S(\theta)\left(g-\varepsilon g_{1}\right)\right) \leqslant C_{1}\left(E_{m-1, k}(u, \theta)+|\varepsilon| E_{m-1, k,[\mu]}(S(\theta) z)\right),
$$

где $C_{1}>0$ - некоторая константа. В самом деле, легко видеть, что $g$ и $g_{1}-$ непрерывные функции на $\mathbb{R}$ со значениями в $H^{(k)}$. Поэтому достаточно установить (7.10). В силу предложения 4.3 имеем

$$
E_{m-1, k}(u, t) \leqslant C_{2} e^{\varkappa|t-\theta|} E_{m-1, k}(u, \theta), \quad t \in \mathbb{R},
$$

где $C_{2}$ и $\varkappa$-положительные константы. Отсюда и из (5.18) следует, что на отрезке $[\theta, \theta+1]$ для функции $g(t, x)$ вьполнено неравенство

$$
\|g(t, \cdot)\|_{(k)} \leqslant C_{3} E_{m-1, k}(u, \theta), \quad \theta \leqslant t \leqslant \theta+1,
$$

а вне отрезка $[\theta, \theta+1]$ она обращается в нуль. Для оценки $g_{1}(t, x)$ воспользуемся теоремой о среднем значении, равномерной ограниченностью производных функции $Q_{\rho}$ и неравенством (7.11). В результате получим

$$
E_{0, k,[\mu]}\left(S(\theta) g_{1}\right) \leqslant C_{4}\left(E_{m-1, k}(u, \theta)+E_{m-1, k,[\mu]}(S(\theta) z)\right) .
$$

Сопоставляя (7.12) и (7.13), приходим к (7.10).

Таким образом, для любой функции $z \in \mathbb{F}_{m-1, k,[\mu]}$ правая часть (7.9) принадлежит пространству $\mathbb{F}_{0, k,[\mu]}$. Поэтому в силу предложения 7.2 при $|\varepsilon| \ll 1$ уравнение (7.9) имеет, притом единственное, решение $w=A(z) \in \mathbb{F}_{m-1, k,[\mu]}$, для которого справедлива оценка

$$
\begin{aligned}
E_{m-1, k,[\mu]}(S(\theta) A(z)) & \leqslant C E_{0, k,[\mu]}\left(S(\theta)\left(g-\varepsilon g_{1}\right)\right) \\
& \leqslant C_{5}\left(E_{m-1, k}(u, \theta)+|\varepsilon| E_{m-1, k,[\mu]}(S(\theta) z)\right) .
\end{aligned}
$$


Покажем теперь, что $A$ - сжимаюший оператор. Для этого возьмем две произвольные функции $z_{1}, z_{2} \in \mathbb{F}_{m-1, k,[\mu]}$, положим $w_{i}=A\left(z_{i}\right)$ и рассмотрим разность $w=w_{1}-w_{2}$. Легко видеть, что она является решением уравнения (5.15) с правой частью

$$
h(t, x)=\varepsilon\left(Q_{\rho}\left(\varepsilon, t, \mathscr{D}(t)\left(\zeta u-z_{2}\right)\right)-Q_{\rho}\left(\varepsilon, t, \mathscr{D}(t)\left(\zeta u-z_{1}\right)\right)\right) .
$$

С помощью теоремы о среднем значении без труда устанавливается неравенство

$$
\|h(t, \cdot)\|_{(k)} \leqslant C_{6}|\varepsilon| E_{m-1, k}\left(z_{1}-z_{2}, t\right)
$$

откуда следует, что

$$
E_{0, k,[\mu]}(S(\theta) h) \leqslant C_{6}|\varepsilon| E_{m-1, k,[\mu]}\left(S(\theta)\left(z_{1}-z_{2}\right)\right) .
$$

В силу (7.8) для решения $w \in \mathbb{F}_{m-1, k,[\mu]}$ уравнения (5.15) справедлива оценка

$$
E_{m-1, k,[\mu]}(S(\theta) w) \leqslant C E_{0, k,[\mu]}(S(\theta) h) .
$$

Сравнивая это неравенство с (7.15), получим, что при $|\varepsilon| \ll 1$ оператор $A$ является сжимаюшим.

Обозначим через $w \in \mathbb{F}_{m-1, k,[\mu]}$ неподвижную точку оператора $A: A(w)=w$. Из (7.14) следует, что она удовлетворяет неравенству

$$
E_{m-1, k,[\mu]}(S(\theta) w) \leqslant C_{7} E_{m-1, k}(u, \theta) .
$$

Покажем, что для функции $v(t, x)$, определенной по формуле (5.16), выполнены все требуемые утверждения.

В самом деле, по построению $v(t, x)$ удовлетворяет уравнению (5.9). Так как $\zeta(t)=0$ при $t \leqslant \theta$, то из (5.16), (7.7) и (7.16) получим, что $v \in \mathbb{F}_{m-1, k,[\mu,-\mu]}$. Поэтому согласно утверждению (ii) $\mathscr{D}(t) v \in \mathscr{M}(t)$ при всех $t \in \mathbb{R}$. Далее, из (5.16) и (7.16) следует, что

$$
E_{m-1, k}(u-v, t)=E_{m-1, k}(w, t) \leqslant C_{7} e^{-\mu(t-\theta)} E_{m-1, k}(u, \theta) \text { при } t \geqslant \theta+1 .
$$

Сопоставляя это неравенство с (7.11) и (7.16), приходим к (7.2).

Таким образом, мы установили, что для любого $\theta \in \mathbb{R}$ найдется решение $v=$ $v_{\theta} \in \mathbb{F}_{m-1, k,[\mu,-\mu]}$, удовлетворяющее (7.2). Остается показать, что $v_{\theta_{1}} \equiv v_{\theta_{2}}$ при любых $\theta_{1}, \theta_{2} \in \mathbb{R}$.

Непосредственная проверка показывает, что разность $w:=v_{\theta_{1}}-v_{\theta_{2}}$ принадлежит пространству $\mathbb{F}_{m-1, k,[\mu,-\mu]}$ и является решением уравнения $(5.15)$ с правой частью

$$
h(t, x)=\varepsilon\left(Q_{\rho}\left(\varepsilon, t, \mathscr{D}(t) v_{\theta_{1}}\right)-Q_{\rho}\left(\varepsilon, t, \mathscr{D}(t) v_{\theta_{2}}\right)\right) .
$$

Из неравенства $(7.2)$ с $v=v_{\theta_{i}}$ следует, что

$$
E_{m-1, k}\left(v_{\theta_{1}}-v_{\theta_{2}}, t\right) \leqslant C_{8} e^{-\mu t} E_{m-1, k}(u, \theta) .
$$

Поэтому $w \in \mathbb{F}_{m-1, k,[\mu]}$. Далее, легко показать, что функция $h$ принадлежит пространству $\mathbb{F}_{0, k,[\mu]}$ и удовлетворяет неравенству

$$
E_{0, k,[\mu]}(h) \leqslant C_{9}|\varepsilon| E_{m-1, k,[\mu]}(w) .
$$


В силу (7.8) имеем

$$
E_{m-1, k,[\mu]}(w) \leqslant C E_{0, k,[\mu]}(h) \leqslant C C_{9}|\varepsilon| E_{m-1, k,[\mu]}(w),
$$

откуда следует, что $w \equiv 0$ при $|\varepsilon| \ll 1$.

(iv) Это утверждение является очевидным следствием гладкости оператора $\mathscr{G}(\varepsilon ; \theta, \cdot)$ и формулы $(5.13)$.

(v) Как было показано в $\S 5$, оператор $\mathscr{R}_{j}$ представим в виде $(5.25)$. Теперь заметим, что первое слагаемое в правой части $(5.25)$ является аналогом оператора $\mathscr{R}_{j}$ для линейного уравнения

$$
P_{\varepsilon}(t, x, \partial) u(t, x)=0 .
$$

Согласно $[3$, теорема 6.4$]$ в случае линейных уравнений имеет место представление

$$
\left.\partial_{t}^{j}\left(G(\varepsilon, \theta) U_{0}\right)\right|_{t=\theta}=\sum_{i=0}^{m_{c}-1}\left(r_{i j}\left(\varepsilon, \theta, x, \partial_{x}\right)+\varepsilon d_{i j}(\varepsilon, \theta)\right) u_{i}(x),
$$

где $U_{0}=\left[u_{0}, \ldots, u_{m_{c}-1}\right]$, операторы

$$
d_{i j}(\varepsilon, \theta): H^{(m-1+k-i)} \rightarrow H^{(m+k-j)}
$$

непрерывно зависят от $\theta$ в сильной операторной топологии, а их нормы ограничены равномерно по $(\varepsilon, \theta)$. Положим

$$
\mathscr{B}_{j}\left(\varepsilon ; \theta, U_{0}\right)=\sum_{i=0}^{m_{c}-1} d_{i j}(\varepsilon, \theta) u_{i}+\left.\partial_{t}^{j}(G(\varepsilon, \theta)[0, \ldots, 0, f])\right|_{t=\theta},
$$

где функция $f$ определяется по формуле (5.24). Представление (5.5) является тривиальным следствием $(5.25),(7.17)$ и (7.19). Непрерывность оператора $\mathscr{B}_{j}$ по $\left[\theta, U_{0}\right]$ и равномерная липшицевость по $U_{0}$ вытекают из аналогичных свойств операторов, входяших в правую часть (7.19). Подробности мы оставляем читателю.

(vi) Воспользуемся формулой (7.19). Так как операторы $d_{i j}(\varepsilon, \theta)$ действуют непрерывно из $H^{(m-1+k-i)}$ в $H^{(m+k-j)}$ (см. (7.18)), то достаточно рассмотреть второе слагаемое в правой части (7.19). Прежде всего заметим, что если функция $q$ не зависит от производных порядка $m-1$, то оператор $Q_{\rho}$ является гладким из $\mathbb{R}_{t} \times \mathbb{E}_{m-1, k}$ в $H^{(k+1)}$, причем все его производные равномерно ограничены. Отсюда и из теоремы 6.1 следует, что при $|\varepsilon| \ll 1$ оператор

$$
\left[\theta, u_{0}, \ldots, u_{m_{c}-1}\right] \mapsto f(t, x)=Q_{\rho}\left(\varepsilon, t, \mathscr{D}(t) \mathscr{G}\left(\varepsilon ; \theta, u_{0}, \ldots, u_{m_{c}-1}\right)\right)
$$

непрерывен из $\mathbb{R}_{\theta} \times \mathbb{E}_{m-1, k}$ в $\mathbb{F}_{0, k+1,[\mu,-\mu]}$, а при любом фиксированном $\theta$ удовлетворяет условию Липшица, причем константа Липшица ограничена равномерно по $(\varepsilon, \theta)$. Далее, при достаточно малых $\varepsilon$ линейный оператор

$$
f \mapsto G(\varepsilon, \theta)[0, \ldots, 0, f]
$$

действует непрерывно из $\mathbb{F}_{0, k+1,[\mu,-\mu]}$ в $\mathbb{F}_{m-1, k+1,[\mu,-\mu]}$. При этом он непрерывно зависит от $\theta$ в сильной операторной топологии, а его норма ограничена равномерно по $(\varepsilon, \theta)$. Из вышесказанного следует, что второе слагаемое в правой части (7.19) также удовлетворяет всем требуемым свойствам. 
7.2. Доказательство теорем 5.1 и 5.2. Зафиксируем произвольное $\rho>0$ и определим искомые операторы $\mathscr{R}_{j}$ как сужение операторов (7.1), построенных в теореме 7.1 , на цилиндр $\mathbb{R} \times \mathbb{B}_{m_{c}-1, k+m_{h}}(\rho)$. Все утверждения теорем 5.1 и 5.2 легко выводятся из теоремы 7.1. Докажем, например, притягивающее свойство.

Пусть решение $u(t, x)$ задачи $(0.3),(1.2)$ определено на всей временной оси, а его фазовая траектория $\mathscr{D}(t) u$ целиком содержится в шаре $\mathbb{B}_{m-1, k}(\rho)$. Тогда $u(t, x)$ является решением задачи (5.9), (5.10) и принадлежит пространству $\mathbb{F}_{m-1, k,[\mu,-\mu]}$. Поэтому согласно утверждению (ii) теоремы $7.1 \mathscr{D}(t) u \in \mathscr{M}(t)$ при всех $t \in \mathbb{R}$. Остается заметить, что многообразие $\mathscr{M}(t, \rho)$, определенное в $(5.2)$, является пересечением $\mathscr{M}(t)$ (см. (5.14)) и шара $\mathbb{B}_{m-1, k}(\rho)$.

Предположим теперь, что $u(t, x)$ - решение задачи $(0.3),(1.2)$ с $J=[\theta,+\infty)$, причем $\mathscr{D}(t) u \in \mathbb{B}_{m-1, k}\left(\rho_{1}\right)$ при $t \geqslant \theta$ для некоторого $\rho_{1}<\rho$. Продолжим функцию $u(t, x)$ на всю временную ось $\mathbb{R}$ как решение задачи $(5.9),(5.10),(1.1)$ с данными Коши

$$
u_{j}(x)=\partial_{t}^{j} u(\theta+0, x), \quad j=0, \ldots, m-1 .
$$

Очевидно, что продолженная функция $u(t, x)$ удовлетворяет всем условиям утверждения (iii) теоремы 7.1. Следовательно, найдется такое решение $v(t, x)$ задачи (5.9), (5.10), что $\mathscr{D}(t) v \in \mathscr{M}(t)$ при всех $t \in \mathbb{R}$, и выполнено (7.2). Теперь заметим, что фазовая траектория $\mathscr{D}(t) v$ содержится в шаре $\mathbb{B}_{m-1, k}(\rho)$ при $t \geqslant T \gg 1$, так как она экспоненциально притягивается к $\mathscr{D}(t) u$ при $t \rightarrow+\infty$. Поэтому при $t \geqslant T$ функция $v(t, x)$ удовлетворяет также уравнению $(0.3)$. Полагая $V_{0}=\mathscr{D}(T) v$, из $(7.2)$ получим (5.3).

Доказательство остальных утверждений мы оставляем читателю.

7.3. Доказательство теоремы 5.3. (i) В силу (5.2), если $\mathscr{D}(t) u \in \mathscr{M}(t, \rho)$ при $t \in J$, имеем

$$
\partial_{t}^{m_{c}} u=\mathscr{R}_{m_{c}}\left(\varepsilon ; t, u, \partial_{t} u, \ldots, \partial_{t}^{m_{c}-1} u\right), \quad t \in J .
$$

Заметим, что остаток $R_{m_{c}}(\varepsilon, y, \tau, \xi)$ от деления полинома $\tau^{m_{c}}$ на $P_{c}(\varepsilon, y, \tau, \xi)$ равен $P_{c}(\varepsilon, y, \tau, \xi)-\tau^{m_{c}}$. Поэтому согласно (5.5) имеем

$\mathscr{R}_{m_{c}}\left(\varepsilon ; t, u, \partial_{t} u, \ldots, \partial_{t}^{m_{c}-1} u\right)=\partial_{t}^{m_{c}} u-P_{c}(\varepsilon, t, x, \partial) u+\varepsilon \mathscr{B}_{m_{c}}\left(\varepsilon ; t, u, \ldots, \partial_{t}^{m_{c}-1} u\right)$.

Подставляя эту формулу в (7.20), получим (5.7).

(ii) Прежде всего заметим, что решение задачи (5.7), (5.8) однозначно определяется данными Коши до порядка $m_{c}-1$ в произвольной точке $\theta \in I$.

В самом деле, пусть $u_{1}, u_{2}-$ два решения задачи (5.7), (5.8), причем $\mathscr{D}_{c}(\theta) u_{1}=$ $\mathscr{D}_{c}(\theta) u_{2}$. Тогда разность $w=u_{1}-u_{2}$ удовлетворяет включениям $(5.8)$, начальным условиям

$$
\partial_{t}^{j} w(\theta, x)=0, \quad j=0, \ldots, m_{c}-1,
$$

и уравнению

$$
P_{c}(\varepsilon, t, x, \partial) w(t, x)=h(t, x),
$$

где $h(t, x)=\varepsilon\left(\mathscr{B}_{m_{c}}\left(\varepsilon ; t, u_{1}, \ldots, \partial_{t}^{m_{c}-1} u_{1}\right)-\mathscr{B}_{m_{c}}\left(\varepsilon ; t, u_{2}, \ldots, \partial_{t}^{m_{c}-1} u_{2}\right)\right)$. 
В силу утверждения (ii) теоремы 5.2 правая часть $h(y)$ принадлежит пространству ${ }^{10} C\left(I, H^{\left(k+m_{h}\right)}\right)$, причем

$$
\|h(t, \cdot)\|_{\left(k+m_{h}\right)} \leqslant C_{1}|\varepsilon| E_{m_{c}-1, k+m_{h}}\left(u_{1}-u_{2}, t\right), \quad t \in I,
$$

где константа $C_{1}>0$ не зависит от $\varepsilon$ и $t$. Воспользуемся оценкой нормы решения задачи Коши для строго гиперболических уравнений (см. [12, лемма 23.2.1]):

$$
E_{m_{c}-1, k+m_{h}}(w, t) \leqslant C_{2} \int_{[\theta, t]}\|h(r, \cdot)\|_{\left(k+m_{h}\right)} d r, \quad t \in I
$$

Сопоставляя (7.21) и (7.22), получим, что $u_{1}(t, x) \equiv u_{2}(t, x)$ при $|\varepsilon| \ll 1$.

Предположим теперь, что интервал $I_{1} \subset I$ удовлетворяет условиям, сформулированным в теореме 5.3 , (ii). Зафиксируем произвольное $\theta \in I_{1}$ и рассмотрим решение $v(t, x)$ задачи $(0.3),(1.1),(1.2)$, где

$$
u_{j}(x)= \begin{cases}\partial_{t}^{j} u(\theta, x), & j=0, \ldots, m_{c}-1 \\ \mathscr{R}_{j}\left(\varepsilon ; \theta, u(\theta, \cdot), \ldots, \partial_{t}^{m_{c}-1} u(\theta, x)\right), & j=m_{c}, \ldots, m-1\end{cases}
$$

По построению начальная вектор-функция $\left[u_{0}, \ldots, u_{m-1}\right]$ лежит на многообразии $\mathscr{M}(\theta, \rho)$. Поэтому в силу локальной инвариантности (см. теорему 5.1) фазовая траектория $\mathscr{D}(t) v$ принадлежит $\mathscr{M}(t, \rho)$ до тех пор, пока она находится в шаре $\mathbb{B}_{m-1, k}(\rho)$. Следовательно, согласно утверждению (i) функция $v(t, x)$ удовлетворяет уравнению (5.7). Более того, в начальный момент времени $t=\theta$ ее данные Коши до порядка $m_{c}-1$ совпадают с данными Коши функции $u(t, x)$. Так как решение задачи $(5.7),(5.8),(5.11)$ единственно, то $u(t, x)$ и $v(t, x)$ совпадают на общей области определения. Отсюда следует, что максимальный интервал $J$, на котором определено решение $v(t, x)$, содержит $I_{1}$ и что $u(t, x)=v(t, x)$ при $t \in I_{1}$. Теорема доказана.

\section{§ 8. Приложение}

В настояшем параграфе собраны некоторые вспомогательные утверждения, использованные в основном тексте.

Лемма 8.1. Пусть $\rho>0,0<\gamma \leqslant 1, a k>n / 2$ u $l \geqslant 0$ - целые числа. Тогда найдется такая константа $C=C(\rho, \gamma, k, l)>0$, что

$$
\left\|Q_{\rho}(\varepsilon, t, U+V)-\sum_{j=0}^{l} \frac{1}{j !}\left(D^{j} Q_{\rho}\right)(\varepsilon, t, U)[V, \ldots, V]\right\|_{(k)} \leqslant C\|V\|_{m-1, k}^{l+\gamma},
$$

где $U, V \in \mathbb{E}_{m-1, k}$, а через $D^{j} Q_{\rho}$ обозначается $j$-я производная функиии $Q_{\rho}$ no $U$.

\footnotetext{
${ }^{10}$ Именно здесь используется тот факт, что функция $q$ не содержит производных порядка $m-1$, и поэтому согласно теореме 5.2 , (ii) областью значений оператора $\mathscr{B}_{m_{c}}$ является пространство $H^{\left(k+m_{h}\right)}$, а не $H^{\left(k+m_{h}-1\right)}$.
} 
ДокАЗАТЕЛЬСТво. Согласно предложению 4.1 оператор

$$
Q_{\rho}(\varepsilon, t, U): \mathbb{R}_{t} \times \mathbb{E}_{m-1, k} \rightarrow H^{(k)}
$$

бесконечно дифференцируем по Фреше, причем все его производные равномерно ограничены. Поэтому при $\gamma=1$ неравенство (8.1) является очевидным следствием формулы Тейлора. Покажем, что в качестве $\gamma$ можно выбрать любое число из интервала $(0,1)$.

Так как $Q_{\rho}(\varepsilon, t, U)=0$ при $\|U\|_{m-1, k} \geqslant \rho$, то можно считать, что $\|U\|_{m-1, k}<\rho$. Предположим сначала, что $\|V\|_{m-1, k} \geqslant 2 \rho$. Тогда $\|U+V\|_{m-1, k} \geqslant \rho$ и неравенство (8.1) следует из равномерной ограниченности производных $D^{j} Q_{\rho}$ :

$$
\left\|\left(D^{j} Q_{\rho}\right)(\varepsilon, t, U)[V, \ldots, V]\right\|_{(k)} \leqslant \text { const }\|V\|_{m-1, k}^{j} .
$$

Пусть теперь $\|V\|_{m-1, k} \leqslant 2 \rho$. В силу формулы Тейлора левая часть (8.1) оценивается сверху через const $\|V\|_{m-1, k}^{l+1}$. Остается заметить, что

$$
\|V\|_{m-1, k}^{l+1} \leqslant(2 \rho)^{1-\gamma}\|V\|_{m-1, k}^{l+\gamma} \text { при }\|V\|_{m-1, k} \leqslant 2 \rho .
$$

Напомним, что для функции $w(t, x)$ мы полагаем $S(\theta) w(t, x)=w(t+\theta, x)$.

ЛЕмма 8.2. Для любых $\rho>0, \mu \geqslant 0$ и челого числа $k>n / 2$ оператор композиции

$$
\mathscr{C}: u(y) \mapsto Q_{\rho}(\varepsilon, t, \mathscr{D}(t) u)
$$

действует непрерывно из $\mathbb{F}_{m-1, k,[\mu,-\mu]}$ в $\mathbb{F}_{0, k,[\mu,-\mu]}$ и удовлетворяет глобальному условию Липшица. Более того, если $u, v \in \mathbb{F}_{m-1, k,[\mu,-\mu]}$, то

$$
E_{0, k,[\mu,-\mu]}(S(\theta)(\mathscr{C} u-\mathscr{C} v)) \leqslant C E_{m-1, k,[\mu,-\mu]}(S(\theta)(u-v))
$$

для любого $\theta \in \mathbb{R}$, причем константа $C=C(k, \rho)>0$ не зависит от $u, v, \theta$ $u \mu$.

ДоКАЗАТЕЛЬСТВО. Из бесконечной гладкости оператора (8.2) и ограниченности его производных следует, что если $u \in \mathbb{F}_{m-1, k,[\mu,-\mu]}$, то $\mathscr{C} u \in \mathbb{F}_{0, k,[\mu,-\mu]}$. Кроме того, в силу формулы о среднем значении,

$$
\left\|Q_{\rho}(\varepsilon, t, \mathscr{D}(t) u)-Q_{\rho}(\varepsilon, t, \mathscr{D}(t) v)\right\|_{(k)} \leqslant \text { const } E_{m-1, k}(u-v, t),
$$

откуда получаем (8.3). 


\section{Список литературы}

1. Бабин А.В. Дробные степени нелинейного аналитического дифференциального оператора // Матем. сб. 1979. Т. 109. № 1. С. 12-45.

2. Былов Б. Ф., Виноград Р. Е., Гробман Д. М., Немыцкий В. В. Теория показателей Ляпунова. М.: Наука, 1966.

3. Волевич Л. Р., Ширикян А.Р. Экспоненциальная дихотомия и экспоненциальное расщепление для гиперболических уравнений // Тр. ММО. 1998. Т. 59. С. 106-149.

4. Гробман Д. М. О гомеоморфизме систем дифференциальных уравнений // ДАН СССР. 1959. Т. 128. № 5. С. 880-881.

5. Далецкий Ю. Л., Крейн М. Г. Устойчивость решений дифференциальных уравнений в банаховом пространстве. М.: Наука, 1970.

6. Коддингтон Э. А., Левинсон $Н$. Теория обыкновенных дифференциальных уравнений. М.: ИЛ, 1958.

7. Мизохата $C$. Теория уравнений с частными производными. М.: Мир, 1977.

8. Николенко H. В. Метод нормальных форм Пуанкаре в задачах интегрируемости уравнений эволюционного типа // УМН. 1986. Т. 41. № 5. С. 109-152.

9. Нитецки З. Введение в дифференциальную динамику. М.: Мир, 1975.

10. Хартман $\Phi$. Обыкновенные дифференциальные уравнения. М.: Мир, 1970.

11. Хенри Д. Геометрическая теория полулинейных параболических уравнений. М.: Мир, 1985.

12. Хермандер Л. Анализ линейных дифференциальных операторов с частными производными. 3. Псевдодифференциальные операторы. М.: Мир, 1987.

13. Albrecht F., Diamond H. G. The converse Taylor theorem // Indiana Math. J. 1971. V. 21. P. 347-350.

14. Bates P., Jones C.K. Invariant manifolds for semilinear partial differential equations // Dynamics Reported. 1989. V. 2. P. 1-38.

15. Carr J. Applications of Center Manifold Theory // Appl. Math. Sci. V. 35. Heidelberg-Berlin-New York: Springer-Verlag, 1981.

16. Chicone C., Latushkin $Y$. Center manifolds for infinite dimensional nonautonomous differential equations // J. Diff. Eq. 1997. V. 141. P. 356-399.

17. Chow S.-N., Lu K., Shen $Y$.-Q. Normal forms for quasiperiodic evolutionary equations // Discrete Contin. Dynam. Systems. 1996. V. 2. № 1. P. 65-94.

18. Foias C., Saut J.-C. Linearization and normal form of the Navier-Stokes equations with potential forces // Ann. Inst. Henri Poincaré. 1987. V. 4. P. 1-47.

19. Haraux A. Semi-Linear Hyperbolic Problems in Bounded Domains. Chur-London-ParisNew York-Melbourne: Harwood Acad. Publ., 1987.

20. Hartman P. A lemma in the theory of structural stability of differential equations // Proc. Amer. Math. Soc. 1960. V. 11. P. 610-620.

21. Kelley A. The stable, center-stable, center, center-unstable, unstable manifolds // J. Diff. Eq. 1967. V. 3. P. 547-570.

22. Kirchgässner K. Wave solutions of reversible systems and applications // J. Diff. Eq. 1982. V. 45. P. 113-127.

23. McKean H. P., Shatah J. The nonlinear Schrödinger equation and nonlinear heat equation. Reduction to linear form // Comm. Pure Appl. Math. 1991. V. XLIV. P. 1067-1080.

24. Mielke A. A reduction principle for nonautonomous systems in infinite-dimensional spaces // J. Diff. Eq. 1986. V. 65. № 2. P. 322-355.

25. Mielke A. Locally invariant manifolds for quasilinear parabolic equations // Rocky Mountain J. Math. 1991. V. 21. № 2. P. 707-714.

26. Mielke A. Essential manifolds for an elliptic problem in an infinite strip // J. Diff. Eq. 1994. V. 110. № 2. P. 322-355.

27. Mora X., Solà-Morales J. Existence and non-existence of finite-dimensional globally attracting invariant manifolds in semilinear damped wave equations // Dynamics of Infinite-Dimensional Systems / Eds. S.-N. Chow and J. K. Hale. NATO ASI. Ser. F. V. 37. New York-Berlin: Springer-Verlag, 1987. P. 187-210. 
28. Palmer K. J. A generalization of Hartman's linearization theorem // J. Math. Anal. Appl. 1973. V. 41. № 3. P. 243-255.

29. Palmer K. J. Linearization near an integral manifold // J. Math. Anal. Appl. 1975. V. 51. P. 243-255.

30. Pugh C. C. On a theorem of P. Hartman // Amer. J. Math. 1969. V. 91. № 2. P. 363-367.

31. Scarpelini A. Center manifolds of infinite dimensions I. Main results and applications; II. Proofs of the main results // Z. Angew. Math. Phys. 1991. V. 42. № 1. P. 1-31; № 2. P. 280-314.

32. Shirikyan A.R., Volevich L. R. Bounded and almost periodic solutions to linear high-order hyperbolic equations // Math. Nachr. 1998. V. 193. P. 137-197.

33. Vanderbauwhede A. Centre manifolds, normal forms and elementary bifurcations // Dynamics Reported. 1989. V. 2. P. 89-169.

34. Vanderbauwhede A., Iooss G. Center manifold theory in infinite dimensions // Dynamics Reported. 1992. V. 1 (new series). P. 125-163.

Поступило в редакцию 19.X.1998 